\title{
THE PdBI ARCSECOND WHIRLPOOL SURVEY (PAWS). I. A CLOUD-SCALE/MULTI-WAVELENGTH VIEW OF THE INTERSTELLAR MEDIUM IN A GRAND-DESIGN SPIRAL GALAXY
}

\author{
Eva Schinnerer ${ }^{1}$, Sharon E. Meidt ${ }^{1}$, Jérôme Pety ${ }^{2,3}$, Annie Hughes ${ }^{1}$, Dario Colombo ${ }^{1}$, \\ Santiago García-Burillo ${ }^{4}$, Karl F. Schuster ${ }^{2}$, Gä̈lle Dumas ${ }^{2}$, Cllare L. Dobbs ${ }^{5}$, Adam K. Leroy ${ }^{6}$, \\ Carsten Kramer $^{7}$, Todd A. Thompson ${ }^{8,9}$, and Michael W. Regan ${ }^{10}$ \\ ${ }^{1}$ MPI for Astronomy, Königstuhl 17, D-69117 Heidelberg, Germany \\ ${ }^{2}$ Institut de Radioastronomie Millimétrique, 300 Rue de la Piscine, F-38406 Saint Martin d'Hères, France \\ ${ }^{3}$ Observatoire de Paris, 61 Avenue de l'Observatoire, F-75014 Paris, France \\ ${ }^{4}$ Observatorio Astronómico Nacional-OAN, Observatorio de Madrid Alfonso XII 3, E-28014 Madrid, Spain \\ ${ }^{5}$ School of Physics and Astronomy, University of Exeter, Stocker Road, Exeter EX4 4QL, UK \\ ${ }^{6}$ National Radio Astronomy Observatory, 520 Edgemont Road, Charlottesville, VA 22903, USA \\ ${ }^{7}$ Instituto Radioastronomía Milimétrica, Avenida Divina Pastora 7, Nucleo Central, E-18012 Granada, Spain \\ ${ }^{8}$ Department of Astronomy, The Ohio State University, 140 West 18th Avenue, Columbus, OH 43210, USA \\ ${ }^{9}$ Center for Cosmology and AstroParticle Physics, The Ohio State University, 191 West Woodruff Avenue, Columbus, OH 43210, USA \\ ${ }^{10}$ Space Telescope Science Institute, 3700 San Martin Drive, Baltimore, MD 21218, USA \\ Received 2013 February 15; accepted 2013 March 27; published 2013 November 25
}

\begin{abstract}
The Plateau de Bure Interferometer Arcsecond Whirlpool Survey has mapped the molecular gas in the central $\sim 9 \mathrm{kpc}$ of $\mathrm{M} 51$ in its ${ }^{12} \mathrm{CO}(1-0)$ line emission at a cloud-scale resolution of $\sim 40 \mathrm{pc}$ using both IRAM telescopes. We utilize this data set to quantitatively characterize the relation of molecular gas (or $\mathrm{CO}$ emission) to other tracers of the interstellar medium, star formation, and stellar populations of varying ages. Using two-dimensional maps, a polar cross-correlation technique and pixel-by-pixel diagrams, we find: (1) that (as expected) the distribution of the molecular gas can be linked to different components of the gravitational potential; (2) evidence for a physical link between $\mathrm{CO}$ line emission and radio continuum that seems not to be caused by massive stars, but rather depends on the gas density; (3) a close spatial relation between polycyclic aromatic hydrocarbon (PAH) and molecular gas emission, but no predictive power of PAH emission for the molecular gas mass; (4) that the $I-H$ color map is an excellent predictor of the distribution (and to a lesser degree, the brightness) of CO emission; and (5) that the impact of massive (UV-intense) young star-forming regions on the bulk of the molecular gas in central $\sim 9 \mathrm{kpc}$ cannot be significant due to a complex spatial relation between molecular gas and star-forming regions that ranges from cospatial to spatially offset to absent. The last point, in particular, highlights the importance of galactic environment—and thus the underlying gravitational potential—for the distribution of molecular gas and star formation.
\end{abstract}

Key words: galaxies: individual (NGC 5194) - galaxies: ISM

\section{INTRODUCTION}

Understanding the distribution and properties of cold molecular gas is fundamental for developing a coherent model for star formation as molecular gas is regarded as the fuel out of which stars form. The need for a physical model on the few parsec to kiloparsec scale has become more urgent as theoretical simulations and models can now incorporate a more complete handling of the gas properties on these scales (e.g., Robertson \& Kravtsov 2008; Gnedin et al. 2009; Christensen et al. 2012). In particular, giant molecular clouds (GMCs) received tremendous attention as most massive star formation in the Milky Way is associated with them (e.g., Mooney \& Solomon 1988; Mead et al. 1990). This clearly suggests a very close link between (massive) star formation and molecular gas, which has also been demonstrated in recent studies of external galaxies to hold at kpc-resolution (Leroy et al. 2013; Bigiel et al. 2008, 2011). However, at smaller scales, it is not exactly clear what is causing or dominating the physical link: small scale physics, like local turbulence within clouds, or large-scale processes such as density waves.

Most of the knowledge in this area is based on our understanding of molecular line emission observed in the Milky Way where the role of galactic environment is harder to study than in nearby galaxies due to the distance ambiguity. In the past few years, there has been mounting evidence that the properties of the molecular gas and GMCs in particular are not necessarily uniform in certain galactic environments (e.g., Bolatto et al. 2008; Gratier et al. 2012; Rosolowsky et al. 2003; Donovan Meyer et al. 2011; Rebolledo et al. 2012; Hirota et al. 2011; Nieten et al. 2006; Espada et al. 2012; Wei et al. 2012). However, none of these studies covered a large enough sample of GMCs or environments within a single galaxy to obtain robust statistics and search for changes of the molecular gas or the environment that could quantify the deviations.

As the most abundant molecule, $\mathrm{H}_{2}$ cannot be directly observed, therefore the line emission of the $\mathrm{CO}$ molecule serves as the most common substitute. Over the past decades, several relations between $\mathrm{CO}$ line emission and line as well as continuum emission at other wavelengths have been observed. While the underlying cause for these relations is often not well understood, studies that aim to test on which spatial scales such relations might exist, i.e., globally versus locally, bear the potential to unravel the physical processes underlying the relations. Several relations between the $\mathrm{CO}$ emission and other tracers of the interstellar medium (ISM), as well as the stellar population, have been established (mostly) on global scales, however, it is unclear if they persist on smaller spatial scales. Prominent examples are the relations between $\mathrm{CO}$ emission and (far-)infrared luminosity (e.g., Young \& Scoville 1991), CO emission and 
Table 1

Basic Parameters for M51 (NGC 5194)

\begin{tabular}{lcc}
\hline \hline Parameter & Value & Comments \\
\hline Hubble type & $\mathrm{SA}(\mathrm{s}) \mathrm{bc} \mathrm{pec}$ & NASA Extragalactic Database \\
Center & $13: 29: 52.7087+47: 11: 42.789$ & $(\mathrm{~J} 2000),(1)$ \\
$v_{\text {sys }}(\mathrm{LSR})$ & $471.7 \pm 0.3 \mathrm{~km} \mathrm{~s}$ & Radio convention, (2) \\
Distance & $7.6 \mathrm{Mpc}$ & $1^{\prime \prime}=36.8 \mathrm{pc},(3)$ \\
Inclination & $22 \pm 5$ & $(4)$ \\
PA & $172 \pm 3$ & $(4)$ \\
Stellar mass $M_{\star}$ & $3.6 \times 10^{10} M_{\odot}$ & $(5)$ \\
Atomic gas mass $M_{\mathrm{H}}$ & $2.8 \times 10^{9} M_{\odot}$ & $(5)$ \\
Molecular gas mass $M_{\text {mol }}$ & $6.2 \times 10^{9} M_{\odot}$ & $(2)$ \\
Cold gas mass $M_{\text {gas }}$ & $9.0 \times 10^{9} M_{\odot}$ & \\
$f_{\text {gas }}=M_{\text {gas }} /\left(M_{\text {gas }}+M_{\star}\right)$ & 0.2 & \\
\hline
\end{tabular}

Notes. Basic parameters adopted for M51 (NGC 5194) and used in all PAWS related publications. References. (1) Hagiwara 2007; (2) Shetty et al. 2007; (3) Ciardullo et al. 2002; (4) Colombo et al. 2013b; (5) Leroy et al. 2008.

radio continuum (RC; e.g., Allen 1992), and last but not least the relation between star formation surface density and neutral gas surface density, i.e., the Kennicutt-Schmidt relation (e.g., Kennicutt 1989, 1998; Bigiel et al. 2008). The exact underlying causes for these relations are not fully understood and several physical processes could lead to such relations. Therefore, observations at high spatial resolution are critical to shed light onto the underlying physical processes that might act at different physical scales and potentially allow for the disentanglement of cause and relation.

In this paper, we combine new observations of the $\mathrm{CO}(1-0)$ line emission at $1^{\prime \prime}$ resolution from the Plateau de Bure Interferometer (PdBI) Arcsecond Whirlpool Survey (PAWS) with existing archival multi-wavelength data ranging from the radio to the ultraviolet (UV) to study the relation between molecular gas as seen in its $\mathrm{CO}$ emission and other galactic components (gas, dust, and stars). The paper is organized as follows. After an introduction of the PAWS survey (Section 2), we describe the data sets used and the steps taken to obtain a common astrometry in Section 3. In Section 4, we present our results based on two-dimensional maps, the polar cross correlation (cc) technique, and a pixel-by-pixel analysis for the various ISM and stellar tracers used. The results are discussed in the context of processes acting and affecting the molecular gas on cloud scales (Section 5) and the implications for changes in the galactic environment are presented in Section 6. We summarize and conclude in Section 7. In Appendix A, we provide a short summary of the relevant molecular gas cooling and heating processes in relation to observations of the $\mathrm{CO}$ emission line. A description of the polar cc technique is given in Appendix B.

This paper is the first of a series describing results from the PAWS survey. The PAWS data products will be publicly available from a dedicated Web page. ${ }^{11}$ Pety et al. (2013) find that about half of the $\mathrm{CO}$ emission arises from a diffuse component that is consistent with a thick molecular disk. In addition, a detailed description of the observations, as well as data reduction, can be found there. Motivated by the results of this paper that show environmental changes in the relations between the $\mathrm{CO}$ emission and other tracers, Hughes et al. (2013b) study the probability distribution functions (PDFs) of the molecular gas as a function of environment and find profound differences between center, arm, and interarm regions. A follow-

\footnotetext{
11 http://www.mpia.de/PAWS; see also http://www.iram-institute.org/EN/ content-page-240-7-158-240-0-0.html
}

up of this result is the work by Colombo et al. (2013a), who identify about 1500 individual GMCs in the PAWS data and find that several GMC properties show a strong dependence on galactic environment and thus large-scale dynamics. A comparative study of GMC properties in M51, M33, and the Large Magellanic Cloud (LMC) by Hughes et al. (2013a) shows that the GMC scaling relations found are very sensitive to methodological biases and that distinct difference in the GMC properties between the three studied galaxies exist. Finally, Meidt et al. (2013) provide an explanation for the varying GMC properties found using dynamical pressure arguments and propose environmental dependences of their star forming capabilities.

\section{MOTIVATION FOR THE PdBI ARCSECOND WHIRLPOOL SURVEY}

Although most stars in the universe have been born in galaxies with typical stellar masses of $M_{\star}=10^{10.6 \pm 0.4} M_{\odot}$ (e.g., Karim et al. 2011), detailed studies of the molecular ISM and GMCs - the entities out of which stars form-have focused on lower stellar mass systems in the Local Group. Studies of more massive spiral galaxies often lack resolution and/or areal coverage to identify statistically significant numbers of GMCs in different galactic environments (e.g., Bolatto et al. 2008; Gratier et al. 2012; Rosolowsky et al. 2003; Donovan Meyer et al. 2011; Rebolledo et al. 2012; Hirota et al. 2011; Nieten et al. 2006). Thus, high spatial and spectral imaging of (a significant part of) the molecular gas reservoir of a typical star forming galaxy has not yet been undertaken.

We chose M51 ("The Whirlpool Galaxy") as the target of our survey as it is one of the closest ( $D \sim 7.6 \mathrm{Mpc}$; Ciardullo et al. 2002) face-on ( $i \sim 22^{\circ}$; Tully 1974; Colombo et al. 2013b) grand-design spirals and one of the most observed galaxies in the sky, resulting in an excellent coverage across the full electromagnetic spectrum. A summary of the basic parameters assumed for M51 is given in Table 1. Over 1000 individual star clusters have been observed and characterized based on Hubble Space Telescope (HST) imaging (e.g., Bastian et al. 2005; Scheepmaker et al. 2007, 2009; Haas et al. 2008; Hwang \& Lee 2008; Chandar et al. 2011) and deep, high-resolution imaging of the galaxy in the RC (Dumas et al. 2011), mid- to far-IR (Calzetti et al. 2005; Mentuch Cooper et al. 2012), $\mathrm{P} \alpha / \mathrm{H} \alpha$ (Scoville et al. 2001), and UV bands (Calzetti et al. 2005) trace young stellar populations with a wide range of age sensitivities 
(e.g., Calzetti et al. 2005; Murphy et al. 2011). In contrast to Local Group galaxies with resolved GMC populations, the molecular gas clearly dominates the ISM over the $9 \mathrm{kpc}$ region that PAWS covers (e.g., Schuster et al. 2007; Leroy et al. 2008) and the molecular gas is concentrated into spectacular spiral arms (e.g., Garcia-Burillo et al. 1993; Helfer et al. 2003; Schuster et al. 2007; Koda et al. 2009). This offers the unique opportunity to relate the physical properties of the molecular gas and its clouds to different galactic environments, especially regarding the galaxy dynamics and radiation field.

The fundamental goal of this large IRAM program is to resolve the $\mathrm{CO}$ emission from M51 on the scales of individual GMCs. This enables a new approach to understanding star formation in galaxies, one centered on studying the physical structures of interest rather than averaging over large areas. The inferred total molecular gas mass in NGC 5194, a large spiral galaxy, is $M_{\text {gas }}=6.2 \times 10^{9} M_{\odot}$. About $60 \%$ of this gas mass-comparable to the total molecular mass of the Milky Way-resides in the PAWS field of view (FoV; Pety et al. 2013). Together with the existing multi-wavelength data, PAWS was designed to provide the data to study the evolutionary sequence for GMCs, place strong constraints on the processes that drive cloud and star formation in disk galaxies, reveal the physical underpinnings of one of the most commonly used scaling relations in extragalactic astronomy (the Schmidt-Kennicutt "law"; Kennicutt 1989, 1998), and add a crucial data point regarding the lifetime of GMCs, a lingering unknown that is critical to understanding the gas-star cycle in galaxies.

These goals resulted in the following considerations for the observations with the IRAM PdBI and the $30 \mathrm{~m}$ single dish telescope.

Sensitivity. The goal of identifying individual GMCs in M51 determined the sensitivity required. In the Milky Way, most $\mathrm{H}_{2}$ is found in clouds with masses in the range $10^{5}-10^{6} M_{\odot}$ (e.g., Sanders et al. 1985; Blitz \& Williams 1999). In order to detect GMCs with $1 \times 10^{5} M_{\odot}$ at $5 \sigma$, a $1 \sigma$ point mass sensitivity $\approx 2 \times 10^{4} M_{\odot}$ is sufficient to recover clouds across this entire range.

Resolution. The typical size of a Milky Way GMC is $\sim 40 \mathrm{pc}$ (Solomon et al. 1987) and thus a comparable spatial resolution is required to identify and distinguish individual clouds. This resolution also marginally resolves the dust lanes observed in the optical in M51 (width $\sim 1^{\prime \prime}$ ).

Area surveyed. The survey area was chosen to incorporate three distinct environmental regions: the center encompassing the bulge plus the star-bursting ring, the spiral arms, and the (relatively quiescent) interarm region. A substantial area in each regime is necessary to build up sufficient statistics on the molecular gas and its GMC populations.

These considerations resulted in imaging of the ${ }^{12} \mathrm{CO}(1-0)$ line emission from the central $\sim 9 \mathrm{kpc}$ of M51 between 2009 August and 2010 June using both IRAM facilities. The global properties adopted for M51 throughout the PAWS survey are summarized in Table 1.

\section{DATA}

In addition to our own new $\mathrm{CO}(1-0)$ observations, we made use of archival data that trace different phases of the ISM and the stellar populations, as well as star formation present in the disk of M51a. Below, we describe in detail where the data sets come from and how they were aligned to a single absolute astrometric frame. A summary of the multi-wavelength data used here is provided in Table 2 .

\subsection{IRAM PdBI+30 m PAWS Data}

We obtained a map of the molecular gas distribution in the central $11 \times 7 \mathrm{kpc}$ of M51 as part of PAWS (PI: Schinnerer). This IRAM Large Program observed a 60 pointing mosaic with the PdBI in all configurations and mapped the full galaxy system with the $30 \mathrm{~m}$ single dish telescope in the ${ }^{12} \mathrm{CO}(1-0)$ line. The resulting data cube of the combined PdBI and $30 \mathrm{~m}$ data (i.e., short spacing corrected) has a resolution of $1^{\prime \prime} .16 \times 0^{\prime \prime} .97$ (PA $73^{\circ}$ ) with a mean rms of $0.4 \mathrm{~K}$ per $5 \mathrm{~km} \mathrm{~s}^{-1}$ wide channel. A detailed description of the data acquisition and reduction is presented by Pety et al. (2013). We also use the moment maps derived from this PAWS data cube (for details, see Colombo et al. 2013b). The conversion from integrated brightness temperature per beam into molecular hydrogen $\left(\mathrm{H}_{2}\right)$ gas surface density per $0^{\prime} .3$ pixel assumed a Galactic conversion factor of $X_{\mathrm{CO}}=2 \times 10^{20} \mathrm{~cm}^{-2} \mathrm{~K}^{-1} \mathrm{~km}^{-1} \mathrm{~s}$.

The PdBI observations consisted of two 30 pointing mosaics that were independently observed between 2009 August and 2010 April. Each pointing within a mosaic was observed between two calibrator cycles ensuring good and similar $u v$ coverage for each pointing as well as the two mosaics. Standard observations of quasars were used for the passband and atmospheric calibration, which was done using the GILDAS software package (Pety 2005). The $3 \mathrm{~mm}$ receivers were tuned to $115.090 \mathrm{GHz}$, corresponding to the redshifted ${ }^{12} \mathrm{CO}(1-0)$ emission line with a local standard of rest velocity of $471.3 \mathrm{~km} \mathrm{~s}^{-1}$. The $30 \mathrm{~m}$ single dish was used in 2010 May to map a $\sim 60$ square arcminute field that contains the entire M51 system. Seven separate regions were observed in position-switch on-the-fly mode; four of those cover the central $300^{\prime \prime} \times 300^{\prime \prime}$, while the remaining three extend this map to the companion and the outer molecular spiral arms. The data were reduced in a standard manner and re-gridded onto a data cube with a pixel size of $4 "$. The final PAWS data cube is obtained from a joint deconvolution of the PdBI and $30 \mathrm{~m}$ data sets. It has 120 channels with $5 \mathrm{~km} \mathrm{~s}^{-1}$ widths and a pixel size of $0^{\prime \prime} 3$ pixel $^{-1}$.

For the analysis presented here, we use a zeroth moment map that was derived using a combination of the dilated mask technique and H i priors (e.g., Appendix B of Pety et al. 2013). A detailed description of the moment calculation is presented by Colombo et al. (2013b). Briefly, a dilated mask is created by identifying emission above a given threshold of $t \sigma$. Starting from these peaks, the identified islands of emission are expanded to include fainter emission above $p \sigma$. If the central velocity of the islands was more than $30 \mathrm{~km} \mathrm{~s}^{-1}$ away from the central $\mathrm{H}_{\mathrm{I}}$ velocity (based on the 6" resolution robust $\mathrm{H}$ i cube from The $\mathrm{H}$ I Nearby Galaxy Survey, THINGS; see Section 3.6), the islands were excluded from the final mask. Due to the high angular resolution, the PAWS $\mathrm{CO}(1-0) 1^{\prime \prime}$ data sample the rising part of the rotation curve much better than the $\mathrm{H}$ i data, causing a lot of real emission to be dropped. Therefore, two masks were necessary to capture significant emission in the disk and the center, i.e., we used $(t, p)$ of $(4,1)$ and $(10,1.5)$ for the disk and center, respectively, and had an $\mathrm{H}$ I prior for the disk and none for the center.

\subsection{HST Archival Data}

To compare our view of the molecular gas with M51's appearance in the optical and near-infrared, we use HST imaging that covers the PAWS FoV. The HST Advanced Camera for Surveys (ACS) Heritage (GO-10452; PI: Beckwith) final reduced and drizzled mosaics (Mutchler et al. 2005) in the F435W 
Table 2

Multi- $\lambda$ Data of M51 Used

\begin{tabular}{|c|c|c|c|c|c|}
\hline$\lambda /$ Filter/Line & Instrument & $\begin{array}{l}\text { Resolution } \\
\left({ }^{\prime \prime} x^{\prime \prime}\right)\end{array}$ & $\begin{array}{l}\text { Pixel Scale } \\
\left(" \text { pixel }^{-1}\right)\end{array}$ & Source & Comments \\
\hline $\mathrm{CO}(1-0)$ & $\mathrm{PdBI}+30 \mathrm{~m}$ & $1.16 \times 0.97$ & 0.3 & PAWS & $\mathrm{PA} \sim 73^{\circ},(1)$ \\
\hline$J$ band & 2MASS & 2.5 & 1 & 2MASS & Used as astrometric reference \\
\hline$B$ band & HST ACS & 0.1 & 0.05 & HST Heritage & Offset of $0 .{ }^{\prime} 1$ and $-00^{\prime \prime} 4,(2)$ \\
\hline$V$ band & HST ACS & 0.1 & 0.05 & HST Heritage & Offset of $0 .{ }^{\prime} 1$ and $-0.4,4$, (2) \\
\hline$I$ band & HST ACS & 0.1 & 0.05 & HST Heritage & Offset of $0^{\prime \prime} 1$ and $-0^{\prime \prime} 4,(2)$ \\
\hline $\mathrm{H} \alpha$ & HST ACS & 0.1 & 0.05 & HST Heritage & Offset of 0.1 and $-0^{\prime \prime} 4,(2)$ \\
\hline$H$ band & HST NICMOS & 0.2 & 0.05 & M. Regan & Offset $0 . .1$ and $-0 .{ }^{\prime \prime} 4$ \\
\hline$i$ band & SDSS & 1.4 & 0.4 & DRS7 & Montage of six tiles (see Section 3.7) \\
\hline $\mathrm{H} \alpha$ & KPNO & $\sim 1.6$ & 0.305 & SINGS & SINGS, (3) \\
\hline $3.6 \mu \mathrm{m}$ & SST IRAC & $1.90^{\mathrm{a}}$ & 0.75 & $\mathrm{~S}^{4} \mathrm{G}$ & (4) \\
\hline $3.6 \mu \mathrm{m}$ & SST IRAC & $1.90^{\mathrm{a}}$ & 0.75 & $\mathrm{ICA}^{\mathrm{b}}$ & (5) \\
\hline $4.5 \mu \mathrm{m}$ & SST IRAC & $1.81^{\mathrm{a}}$ & 0.75 & $S^{4} G$ & (4) \\
\hline $5.8 \mu \mathrm{m}$ & SST IRAC & $2.11^{\mathrm{a}}$ & 0.75 & SINGS & DR5, (3) \\
\hline $8.0 \mu \mathrm{m}$ & SST IRAC & $2.82^{\mathrm{a}}$ & 0.75 & SINGS & DR5, (3) \\
\hline $24 \mu \mathrm{m}$ & SST MIPS & $6.43^{\mathrm{a}}$ & 1.5 & SINGS & DR5, offset of 0 '.25 and $0 .{ }^{\prime \prime} 5$, , (3) \\
\hline $24 \mu \mathrm{m}$ & SST MIPS & $\sim 2.4$ & 0.4 & HiRes & Offset of $0 . ' 25$ and $0^{\prime \prime} 5,(6)$ \\
\hline $70 \mu \mathrm{m}$ & SST MIPS & $18.74^{\mathrm{a}}$ & 4.5 & SINGS & DR5, Decl. offset of 0!375, (3) \\
\hline $160 \mu \mathrm{m}$ & SST MIPS & $38.78^{\mathrm{a}}$ & 9.0 & SINGS & DR5, Decl. offset of 0!'375, (3) \\
\hline $70 \mu \mathrm{m}$ & $H S O^{\mathrm{c}}$ PACS & 5.67 & 1.0 & $H S O$ archive & Offset of -2 .'5 and -2 .. $0,(7)$ \\
\hline $160 \mu \mathrm{m}$ & HSO PACS & $11.18^{\mathrm{a}}$ & 1.0 & $H S O$ archive & Offset of $-22^{\prime \prime} 5$ and -2 .'0, (7) \\
\hline $\mathrm{C}_{\mathrm{II}}$ & HSO PACS & $\sim 11.5$ & 1.762 & $H S O$ archive & Offset of $2^{\prime \prime} .64$ and $-1^{\prime \prime} .76,(8)$ \\
\hline FUV & GALEX & $4.48^{\mathrm{a}}$ & 1.5 & archive & Offset of $00^{\prime \prime} 225$ and $0 \prime^{\prime} 525,(9)$ \\
\hline NUV & GALEX & $5.05^{\mathrm{a}}$ & 1.5 & archive & Offset of $0^{\prime \prime} .225$ and $0^{\prime \prime} \cdot 525,(9)$ \\
\hline $3.6 \mathrm{~cm}$ & VLA+Eff & 2.4 & 0.4 & & (6) \\
\hline $6 \mathrm{~cm}$ & VLA+Eff & 2.0 & 0.4 & & (6) \\
\hline $20 \mathrm{~cm}$ & VLA+Eff & 1.4 & 0.4 & & (6) \\
\hline $\mathrm{HI}$ & VLA & $5.78 \times 5.56$ & 1.5 & THINGS & $\mathrm{PA}-68.0,(10)$ \\
\hline
\end{tabular}

Notes. Overview of the data used here and astrometric modification applied. See the text for details on the astrometric corrections (Section 3.7) and on the source and processing of the data in Section 3.

a Aniano et al. (2011).

b Independent component analysis.

${ }^{\mathrm{c}}$ Herschel Space Observatory.

References. (1) Pety et al. 2013; (2) Mutchler et al. 2005; (3) Kennicutt et al. 2003; (4) Sheth et al. 2010; (5) Meidt et al. 2012; (6) Dumas et al. 2011; (7) Mentuch Cooper et al. 2012; (8) courtesy of K. Croxall; (9) courtesy of F. Bigiel; (10) Walter et al. 2008.

( $B$-band), F555W ( $V$-band), F658N (H $\alpha$ narrow-band), and F814W (I-band) filters were obtained from the dedicated Web page. ${ }^{12}$ No further processing was applied to the combined mosaics. To obtain a stellar continuum corrected map of the $\mathrm{H} \alpha$ (plus N II) line emission, we followed the approach of Gutiérrez et al. (2011) by subtracting a linear combination of F555W and F814W scaled by 0.0878 from the F658N narrow-line filter that contains the $\mathrm{H} \alpha$ line. Note that this leads to an over-subtraction in the central $30^{\prime \prime}$, which is likely caused by the stellar bulge having a different spectral energy distribution than the disk.

An HST I - H color map was obtained from the ACS Heritage observations and dedicated NICMOS $H$-band observations in the F160W filter (GO-10501; PI: Chandar). The NICMOS observations were a set of observations at 18 positions. We dithered between two locations at each position. The NICMOS data were reduced in a standard manner. Because the NICMOS observations were made using three different guide stars, their relative astrometry is not consistent. We first corrected for the rotation angle offsets between visits using stars that were common between the ACS image and the NICMOS images. We then solved for the positional offset between the NICMOS images and the ACS mosaic by cross-correlating unsharp masked versions of the NICMOS images with the ACS images. We applied these determined offsets to each NICMOS image

12 http://archive.stsci.edu/prepds/m51/ and used the standard DRIZZLE process to combine the images to form a mosaic. We formed an F814W-F160W $(I-H)$ color map by first smoothing the ACS F $814 \mathrm{~W}$ image to the resolution of the NICMOS F160W image and then dividing the F814W image by the F160W image.

\subsection{Spitzer Archival Data}

M51 was imaged from the near- to far-infrared by all Spitzer instruments as part of the SINGS Legacy project (Kennicutt et al. 2003). The Spitzer IRAC $3.6 \mu \mathrm{m}$ and $4.5 \mu \mathrm{m}$ images were reprocessed by the $S^{4} G$ project (Sheth et al. 2010). Meidt et al. (2012) applied and developed a separation method of the stellar and non-stellar components present in these IRAC images based on Independent Component Analysis that requires two adjacent IRAC bands as the only input. We utilized both the map of nonstellar emission at $3.6 \mu \mathrm{m}$ and the contaminant-free old stellar light from Meidt et al. (2012). We used these maps of the old stellar light to subtract the stellar contribution to the $8 \mu \mathrm{m}$ image from the SINGS DR5. ${ }^{13}$

To trace the recent star formation, we used the Spitzer MIPS $24 \mu \mathrm{m}$ image from SINGS DR5 processed with the HiRes algorithm, as described in detail by Dumas et al. (2011), resulting in an increased angular resolution of $\sim 2$ ". 4 .






\subsection{Herschel Archival Data}

M51 has been imaged by PACS onboard Herschel as part of a Guaranteed Time Observations (GTO) program (PI: Wilson; see also Mentuch Cooper et al. 2012). The archival photometric PACS data at 70 and $160 \mu \mathrm{m}$ were reduced to level one using HIPE v6.0 and we then used SCANAMORPHOS v12.0 (Roussel 2013) to produce the final maps. As HIPE v6.0 was used, we converted the PACS images from flight model (FM), 5 to FM, 6 by dividing by the factors listed in the PACS Photometer Point Source Flux Calibration Report v1.0, which are $1.119(70 \mu \mathrm{m})$ and $1.174(160 \mu \mathrm{m})$. All images were converted to $\mathrm{MJy} \mathrm{s}^{-1}$. The mean full width at halfmaximum (FWHM) of the PACS point response functions are $\sim 5^{\prime \prime}$. $(70 \mu \mathrm{m})$ and $\sim 11^{\prime \prime} .4(160 \mu \mathrm{m})$. The actual images use pixel sizes of $1^{\prime \prime}$.

The [C II] emission line has been imaged as well as part of this Herschel GTO program (PI: Wilson; Parkin et al. 2013). The version used here was reduced using the KINGFISH pipeline for PACS spectroscopy (Kennicutt et al. 2011) in HIPE v9.0.3063 using the calibration files in PACS calVersion 41. This is similar to the standard un-chopped pipeline, but includes some modifications for improved handling of transients in the data. In addition to the calibration steps described in detail by Croxall et al. (2012), the latest pointing calibration and the newest drizzle algorithms were used to project these data using HIPE 10.0.2538. The final angular resolution is $\sim 11^{\prime \prime} .5$.

\subsection{GALEX Archival Data}

The far-UV (FUV) and near-UV (NUV) images are from General Investigator (GI) program GI3-050 (Bigiel et al. 2010, and kindly provided to us by PI: Bigiel). M51 was observed for $\sim 10 \mathrm{ks}$ and details of the data reduction are provided in Bigiel et al. (2010). GALEX simultaneously images in the FUV (1350-1750 ̊) and NUV (1750-2800 ̊̊) with an angular resolution (FWHM) of 4".0 and 5".6, respectively, and a FoV of 1.25 (Morrissey et al. 2005).

\subsection{THINGS H I Data}

For comparison with the atomic gas, we use the robust weighted intensity map from THINGS (Walter et al. 2008). The intensity map has an angular resolution of 5.78 $\times 5$ 5'.56 (PA -68.0) and fully covers M51a.

\subsection{Absolute Astrometry and Alignment}

Unlike radiointerferometric images that naturally come with an absolute astrometry, UV/optical/IR images need to be tied to an external astrometric frame. Therefore, we checked and, if necessary, corrected their astrometry, as detailed below. A summary of our corrections applied is provided in Table 2.

We used the Very Large Array (VLA) $20 \mathrm{~cm}$ image of Dumas et al. (2011) as our absolute astrometric reference since it encompasses the entire M51 galaxy system with its angular extent of $\sim 10^{\prime}$. Comparison with the 2MASS $J$-band image and a montage of the SDSS $i$-band tiles (six cover the full M51 system and are combined using the IRSA Montage too ${ }^{14}$ ) obtained from the respective archives suggests that the agreement of (extragalactic) point sources within the M51 system and around it is good. We find small non-systematic offsets between the $J$-band image and the $i$-band montage and therefore decided to use the $J$-band image as our astrometric reference for the optical/near-infrared images since it also has the same geometric projection as the radio data. The final absolute astrometry

\footnotetext{
14 http://montage.ipac.caltech.edu/
}

in 2MASS is $\leqslant 0$ '. 1 depending on the source brightness. ${ }^{15} \mathrm{Be}$ fore deriving the absolute astrometry, we changed the projection of all images to the standard radio interferometric system, i.e., coordinate description (CD) matrix with rotation into CRPIX/ CDELT/CRVAL description without rotation, using the GIPSY task fitsreproj (van der Hulst et al. 1992; Vogelaar \& Terlouw 2001).

As some of the stars present in the images are moving during the period over which the different data sets were obtained, we used a combination of point sources (Galactic stars and stars/unresolved clusters within M51) using WCSTools (Mink 1999) ${ }^{16}$ and the overall geometry of NGC 5194 and NGC 5195 to obtain our best alignment across the different wavelengths. We estimate that our final astrometry is good to about 0 '. 15 for the high-resolution HST images and slightly larger for the lowerresolution IR images. In any case, we do not expect any profound systematic effects in our analysis (particular those done at 3."0 resolution) due to this small error. A detailed discussion of the astrometric accuracy of the PAWS $\mathrm{CO}(1-0)$ data is given by Pety et al. (2013).

We verified that the relative astrometry of the $H S T$ images was correct. Comparison with the 2MASS $J$-band image showed that the HST Heritage I-band image (convolved to the same resolution as the $J$-band image) is offset from the $J$-band image in both R.A. and decl. by $0{ }^{\prime \prime} 1$ and $-0{ }^{\prime \prime} 4$, respectively. We verified this determined offset by comparing the convolved HST ACS $I$-band image with the SDSS $i$-band montage. The same offset was applied to all other HST ACS images as well as the HST NICMOS $H$-band image that had its relative astrometry tied to the ACS I-band image. Note that Chandar et al. (2011) find a similar offset from a comparison with Chandra X-ray imaging.

The absolute astrometry of the IRAC images was found to agree very well with the 2MASS $J$-band image, thus no correction was applied. We used a convolved version of the IRAC $8 \mu \mathrm{m}$ image to test the absolute astrometry of the MIPS $24 \mu \mathrm{m}$ image and find a small offset of 0.'25 and 0.'5 in R.A. and decl. that we corrected. The same offset was applied to the HiRes version of the MIPS $24 \mu \mathrm{m}$ image. Iteratively, we checked the absolute astrometry of MIPS $70 \mu \mathrm{m}$ and MIPS $160 \mu \mathrm{m}$ images using the corrected MIPS $24 \mu \mathrm{m}$ and MIPS $70 \mu \mathrm{m}$ images as references, respectively. Again, a small offset of 0.'375 in declination was found and corrected.

For the Herschel PACS $70 \mu \mathrm{m}$ and $160 \mu \mathrm{m}$ data, we determined the offset based on the corrected MIPS $24 \mu \mathrm{m}$ image (plus the MIPS $70 \mu \mathrm{m}$ image for the PACS $160 \mu \mathrm{m}$ map) and found small offsets of -2 ".5 and -2 ". 0 that we corrected. The absolute astrometric uncertainty for the [C II] line map is larger $\left(\sim 1^{\prime \prime}\right)$ as the centering was achieved using both the MIPS $24 \mu \mathrm{m}$ image as well as the SINGS H $\alpha$ image convolved to $3^{\prime \prime}$. However, obvious common structures cannot be easily identified between these maps leading to the higher uncertainty.

The astrometry of the GALEX NUV and FUV images was checked against a corrected and convolved version of the IRAC $8 \mu \mathrm{m}$ image as the overall morphology of these tracers is very similar. In particular, coincident point sources were utilized to determine an offset of 0.'225 and 0.525 in R.A. and declination that was corrected.

\footnotetext{
15 See the "Explanatory Supplement to the 2MASS All Sky Data Release and Extended Mission Products" at http://www.ipac.caltech.edu/2mass/releases/ allsky/doc/explsup.html.

16 http://tdc-www.harvard.edu/wcstools/
} 

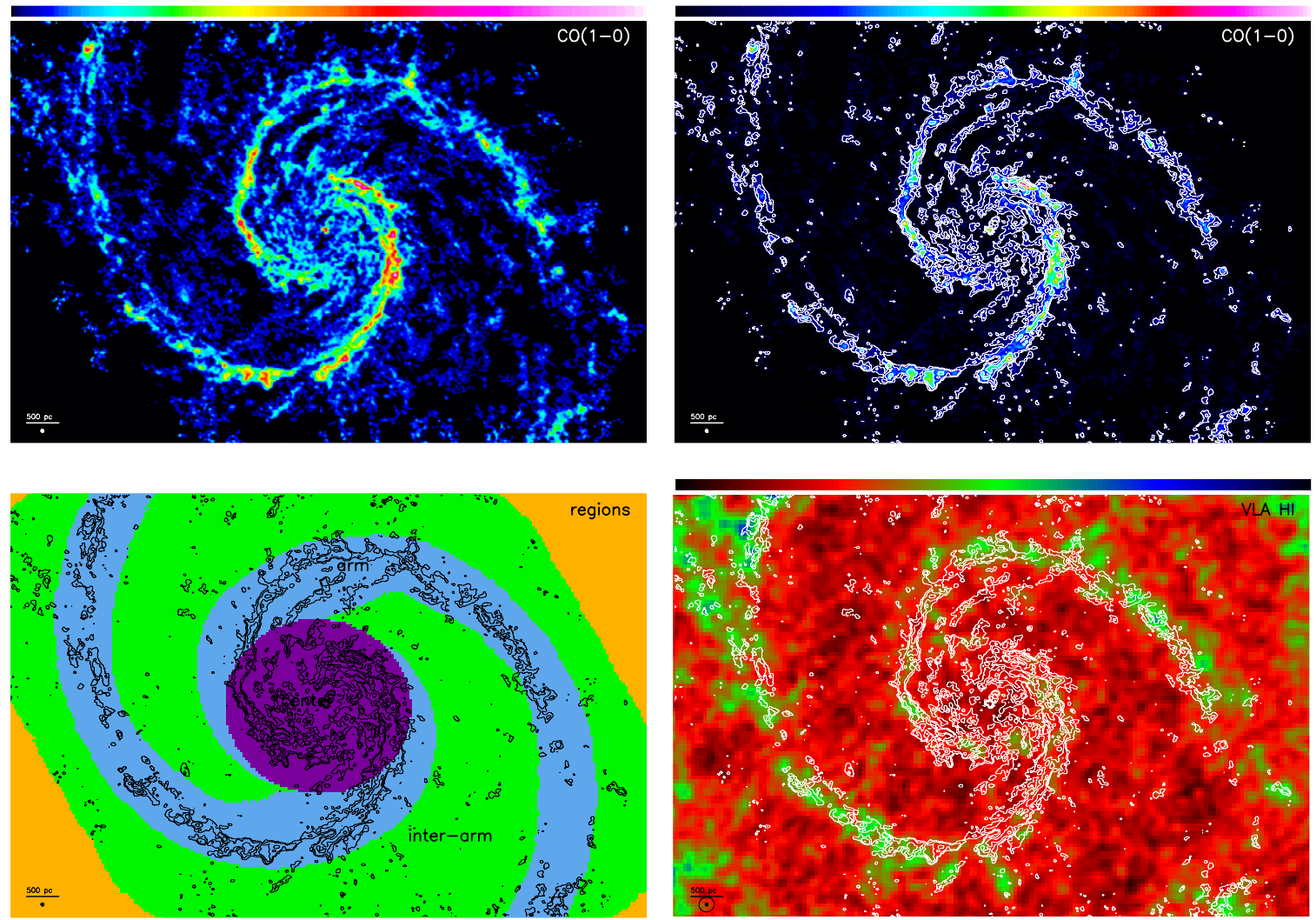

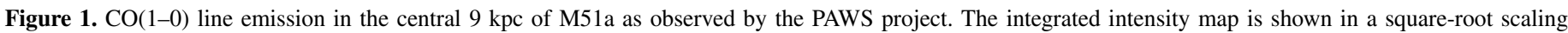



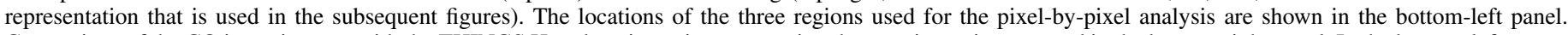

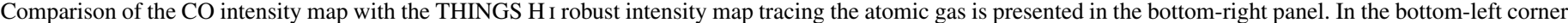
of each panel, a scale bar representing $500 \mathrm{pc}$ at the assumed distance of M51 and the CLEAN beam of the CO(1-0) data are shown.

\section{COMPARISON OF CO(1-0) WITH ISM AND STELLAR TRACERS}

The study of potential relationships between molecular gas line emission, namely $\mathrm{CO}$, and other tracers of the ISM and stellar populations from various ages at cloud scales can provide new insights in the underlying physical processes linking these emissions. In particular, the wide range of galactic environments (i.e., bulge/disk, spiral arm/interarm) sampled by the PAWS FoV could help to discriminate between different origins for existing correlations. After a description of the $\mathrm{CO}$ emission in the central $\sim 9 \mathrm{kpc}$ as seen by PAWS (Section 4.1 ), we evaluate the correspondence between CO emission and the ISM (Section 4.2) and stellar (Section 4.3) tracers in this section. In Section 5, we discuss potential physical causes for the relations and deviations seen. All the findings are placed in the wider context of galactic environment in Section 6.

In the following, we compare the distribution and intensity of the $\mathrm{CO}(1-0)$ molecular line emission with other prominent ISM tracers such as the atomic gas emission lines, optical extinction, hot dust and polycyclic aromatic hydrocarbons (PAHs) emission, and RC. In order to study the relation of the molecular gas to recent and former star formation, we compare the distribution of the $\mathrm{CO}(1-0)$ line emission to line emission and thermal $\mathrm{RC}$ of $\mathrm{HII}$ regions, the light from (young) stellar clusters, the UV continuum from young to intermediate aged stars ( 100 Myr), as well as the light emerging from the old stellar population. In order to capture the different aspects of the relationship between these tracers, we present the data in classical two-dimensional maps using the images at their native resolutions, a polar projection including a cc analysis, and a pixel-by-pixel analysis after convolving all data to a common resolution of 3 .' 0 .

The polar coordinate system is very powerful for studies of spiral arms as it allows for an easy assessment of their properties. We use a linear $4 \pi$ presentation of the azimuthal angle (with " 0 " corresponding to west and a counter-clockwise direction) and a linear projection for the radius. Before re-projection, all images were convolved to the resolution of the 3".0 PAWS data cube and re-gridded to its pixel scale. We converted all flux scales to units of "per pixel area." The polar cc allows for a quantitative assessment of azimuthal offsets between two different tracers (see Appendix B for details). With this definition, a $\Phi>0$ offset for, e.g., star formation tracers, implies that gas is flowing through the spiral mode inside corotation for a given perturbation to the gravitational potential. Several patterns (or perturbations) have been identified in the disk of M51 with different pattern speeds (e.g., Meidt et al. 2008; Vogel et al. 1993; Elmegreen et al. 1989; Tully 1974). A detailed analysis of the various patterns and their associated pattern speeds (see the Appendix of Meidt et al. 2013) suggests that at any given radius (probed by the PAWS FoV) one is inside a corotation resonance and thus the expected reversal of gas flow beyond the corotation resonance expected for a single pattern is not observed.

To illustrate the differences between the different tracers and the $\mathrm{CO}$ emission more quantitatively, we utilize pixel-by-pixel diagrams plus an additional environmental mask separating the central disk from the spiral arms and the interarm region (Figure 1, bottom left). The density of the data points is 
presented on a logarithmic scale. Where appropriate, we fit a linear relation using the IDL procedure ROBUST_LINEFIT that performs a best straight-line fit accounting the errors in both coordinates as weights for the data point. Lacking error maps for the different tracers, we use the same weight of unity (rather than an error) for all data points/pixels.

\subsection{The Cloud-scale Molecular Gas Distribution in M51 as Seen via $\mathrm{CO}(1-0)$}

The $\mathrm{CO}(1-0)$ line distribution exhibits two prominent spiral arms with the northern arm being on average fainter than the southern arm (Figure 1, top). After winding for about $180^{\circ}$, both gas spiral arms become significantly less prominent and even indistinguishable from the interarm in the case of the northern arm. In addition, the southern arm shows a bifurcation exactly south of the nucleus that persists for larger radii. On the other hand, the northern spiral arm shows a pronounced "dent" northeast of the nucleus. Taken together, this strongly suggests that the spiral arms do not represent a single pattern. The two spiral arms emerge from a ring-like distribution close to the center. The disk inside this ring shows fainter emission distributed in abundant smaller structures. $\mathrm{CO}$ emission is present between the spiral arms, however, at an even fainter level and in a more flocculent fashion.

In the polar representation, the $\mathrm{CO}(1-0)$ spiral arms are almost straight lines as expected for a log-normal spiral (Figure 4, top left). However, close inspections reveal three breaks with differing prominence for both arms at radii of $\sim 27^{\prime \prime}, \sim 55^{\prime \prime}$, and $\sim 90^{\prime \prime}$ with observed pitch angles of about $40^{\circ}, 30^{\circ}$, and $10^{\circ}$, respectively. In addition, an azimuthal offset along the spiral arm at $r \approx 35^{\prime \prime}$ is most evident in the northern spiral arm. The overall slope of the two spiral arms is not identical, suggesting that the underlying gravitational potential cannot be described by a pure $m=2$ mode. The molecular ring appears as an almost horizontal line for radii of $15^{\prime \prime}<r<20^{\prime \prime}$. Below a radius of $\sim 10^{\prime \prime}$, the sampling in azimuth is becoming too sparse in our presentation of the data to draw any firm conclusions. The difference in spiral arm pitch angle has already been noted before by Henry et al. (2003), who analyzed the BIMA SONG data (Helfer et al. 2003) and reported "break radii" of 27 " and 60". The latter break at $60^{\prime \prime}$ is close to our inferred break at $55^{\prime \prime}$. The discontinuity at $35^{\prime \prime}$, on the other hand, appears strongly only at the high resolution of our data and is absent at the low resolution of the BIMA SONG map (e.g., Figures 1 and 2 of Henry et al. 2003). It is interesting to note that the radial profile of the azimuthally averaged torques (see Figure 1 of Meidt et al. 2013) changes direction at radii of $20^{\prime \prime}$ (corotation resonance of the nuclear bar), 35" (start of spiral pattern), 55" (corotation resonance of spiral pattern), and $90^{\prime \prime}$ (start of second spiral pattern), consistent with the breaks observed in the $\mathrm{CO}$ distribution at $35^{\prime \prime}, 55^{\prime \prime}$, and $90^{\prime \prime}$. Using a banana wavelet analysis, Patrikeev et al. (2006) find values similar to ours for the pitch angles of the $\mathrm{CO}$ spiral arms and show that the pitch angles of the two spiral arms differ by on average $10^{\circ}$ over the PAWS FoV. Henry et al. (2003) suggested the presence of an additional $m=3$ mode in the central $6 \mathrm{kpc}$ that could explain both the angular offset between the two gaseous arms and their asymmetry in intensity.

\subsection{Comparison of $\mathrm{CO}(1-0)$ with Other ISM Tracers}

Two other tracers of neutral gas can be compared with the distribution of molecular gas at angular resolutions of $\sim 6^{\prime \prime}$, the atomic gas in its $\mathrm{H}$ I line emission and from the strongest cooling line of the neutral gas, and [C II] at $158 \mu \mathrm{m}$ as imaged by PACS. Three different types of tracers of the dust in a galaxy have sufficient angular resolution for a detailed comparison with the CO maps: (1) an optical extinction map from HST, (2) maps of PAH emission from Spitzer, and (3) the hot dust heated by young stars. We expect varying degrees of correlation between the molecular gas distribution and these dust maps, given that excitation conditions likely vary across our FoV. While the cold dust is best traced at long infrared wavelengths (e.g., Dale et al. 2012; Aniano et al. 2012), available maps have a fairly low angular resolution of, e.g., $\geqslant 12^{\prime \prime}$ for $210 \mu \mathrm{m}$ onward for the Herschel SPIRE instrument. The non-thermal RC is probing very different components related to the ISM and it can have several origins (supernovae (SNe), stellar remnants, radio jets, magnetic fields, etc.).

\subsubsection{Neutral Gas}

The strongest far-IR cooling line of the cold ISM in nearby galaxies is [C II] at $158 \mu \mathrm{m}$, which can contain up to $1 \%$ of the total far-IR luminosity (Stacey et al. 1991; Brauher et al. 2008; Gracia-Carpio et al. 2011). Typically most of the [C II] emission in nearby galaxies arises from the surface layer of molecular clouds where the gas is warm, dense, and photodissociated by FUV emission from either nearby hot (OB) stars and/or the interstellar radiation field (ISRF; see, e.g., the introduction of Stacey et al. 2010). Comparison with the $\mathrm{CO}(1-0)$ emission (Figure 2, top left) shows a close correspondence between the peaks of both emission lines in the molecular ring out to a radius of $\sim 20^{\prime \prime}$, except for a region in the northeast segment of the [C II] ring that has no counterpart in the $\mathrm{CO}(1-0)$ emission. Between the ring and the spiral arms, the [C II] emission exhibits gaps, similar to the ones present in the host dust emission. Along the spiral arms, the most intense [C $\mathrm{II}]$ emission is patchy and is offset to the convex side of the molecular gas spiral arms.

The robustly weighted intensity map of the THINGS data that images the distribution of the atomic gas shows very little pronounced structure in the central region of M51; a few peaks coincide with the molecular gas ring (Figure 1, bottom right). Along the molecular spiral arms, the $\mathrm{H}$ I line emission becomes stronger, reaching its maximum (within the PAWS FoV) roughly at the same locations where hot dust emission is observed. Taken together, the distributions of [C II] and $\mathrm{H}$ I strongly suggest that along the spiral arms both emission lines trace the dissociation process of molecular clouds caused by the massive young star formation. Within the molecular ring, the situation is more complex, as the [C $\mathrm{II}]$ emission is strongest, but no substantial $\mathrm{H}$ I emission is seen there.

\subsubsection{Optical Extinction}

According to results for local Galactic clouds (e.g., Dickman 1978; Lada et al. 2007), maps of (optical) extinction are a strong proxy for the cold dust distribution. Comparison to the $H S T I-H$ image (Figure 2, top right) shows that the optical extinction is a very good predictor of the location of strong $\mathrm{CO}(1-0)$ emission and its underlying detailed fine-structured morphology. This is most obvious in the spiral arms. In the interarm region, the correlation is less pronounced, which is due in part to the presence of stellar clusters along spurs (see La Vigne et al. 2006; Chandar et al. 2011) that are especially prominent in the bluer bands observed with the HST (see Section 4.3.3).

The polar cc of these two tracers shows no azimuthal offset nor any radial trend (Figure 5, top right), consistent with both tracers 

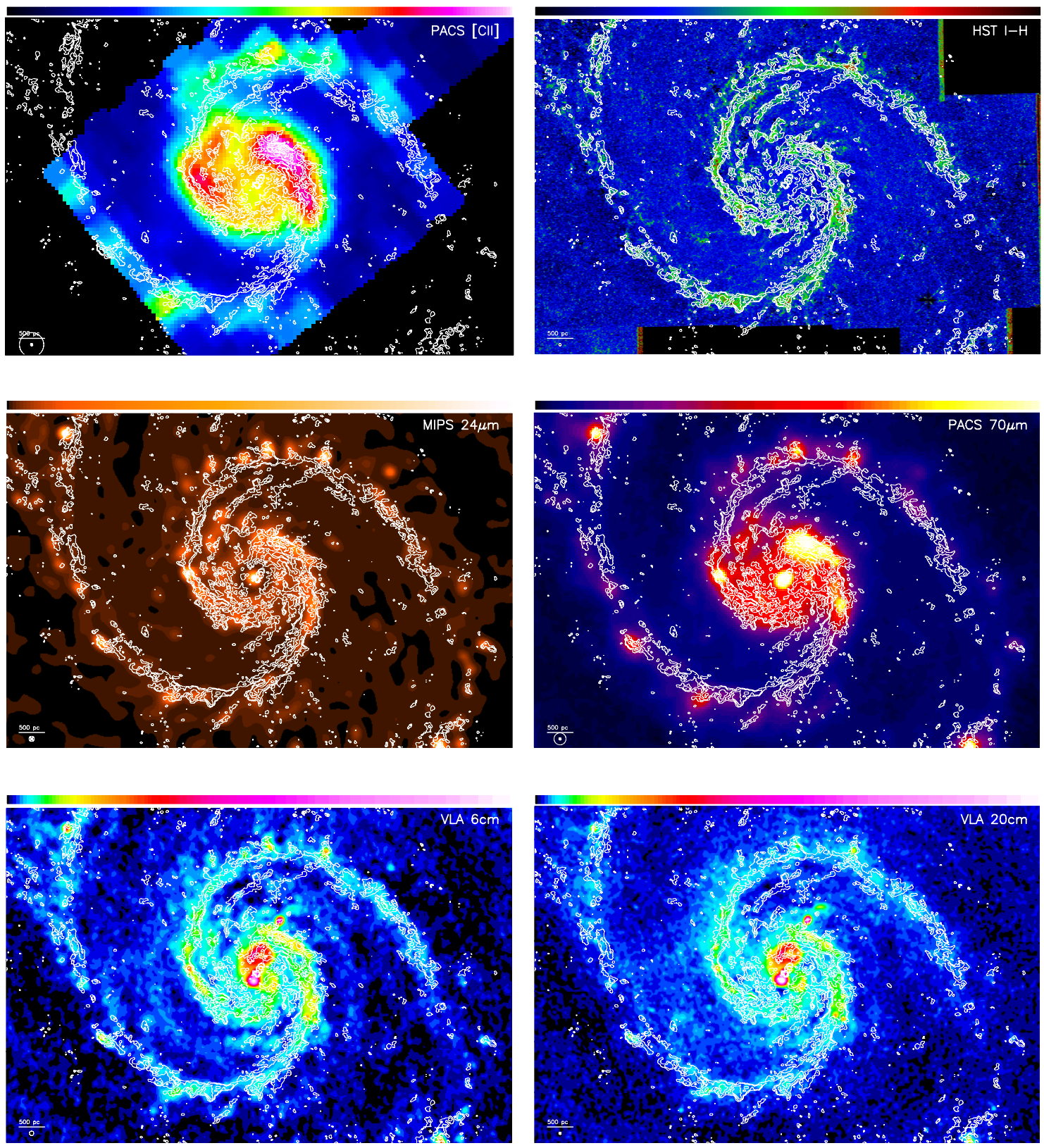

Figure 2. Comparison of the $\mathrm{CO}(1-0)$ line emission in the central $9 \mathrm{kpc}$ of M51a as observed by the PAWS project overlaid in contours (same as in Figure 1, top right) on the Herschel [C II] line map tracing photodissociation (top left), the HST I-H color image tracing extinction (top right), the MIPS HiRes $24 \mu \mathrm{m}$ image tracing hot dust emission (middle left), the PACS $70 \mu \mathrm{m}$ image tracing warm dust emission (middle right), the VLA $6 \mathrm{~cm}$ image tracing thermal and non-thermal RC (bottom left), and the VLA $20 \mathrm{~cm}$ image tracing mainly non-thermal RC (bottom right). In the bottom-left corner of each panel, a scale bar representing $500 \mathrm{pc}$ and the CLEAN beam of the $\mathrm{CO}(1-0)$ data, as well as the resolution of the respective data set, are shown.

being spatially coincident. The prominent (dust) clump in the interarm at an azimuthal angle of $\sim 240^{\circ}$ and a radius of $\sim 39^{\prime \prime}$ has a CO counterpart at a lower level. Inspection of the $I$ - and $\mathrm{H}$-band images suggest that the color of this clump is affected by a group of star clusters causing this apparent inconsistency.

Overall, the molecular gas distribution above an $\mathrm{H}_{2}$ surface density $\Sigma_{M_{\mathrm{H}_{2}}}$ level of $\sim 90-100 M_{\odot} \mathrm{pc}^{-2}$ is well predicted by the $I-H$ color map. Detailed comparison on a pixel-by-pixel basis at 3 .'0 resolution shows that the relation between the HST I-H color and the $\mathrm{CO}$ integrated intensity is linear before it appears to saturate (Figure 6, top row). While this trend is present in all environments, the slope in the central region is significantly shallower, suggesting that the parameters controlling the relation are changing. The similar slope for the arm and interarm regions means that the gas-to-dust ratio is not dramatically changing between the arm and interarm.

\subsection{3. (Hot) Dust Emission}

The distribution of the emission from hot dust probed at $24 \mu \mathrm{m}$ and $70 \mu \mathrm{m}$ by the Spitzer MIPS and Herschel PACS instruments shows a bright central disk encompassing the molecular gas ring (Figure 2, middle row). In addition, several bright peaks are detected along the molecular spiral arms with a slight azimuthal offset of $\Phi>0$ (measured counter-clockwise) from the convex side of the spiral arm and an apparent gap close to the inner disk. The active nucleus in M51a stands out as a 
bright point source in the IR maps. Low-level (mid-)IR emission is seen in the interarm region (about one order of magnitude fainter than the peaks). While the spatial coincidence between the $\mathrm{CO}(1-0)$ and hot dust emission is very good for the central disk, it starts to vanish for the spiral arms, especially when only the high surface brightness regions are considered.

The polar representation (Figure 4, middle left) clearly reveals gaps between the central disk and the spiral arms: in the northern spiral arm, the gaps are slightly more prominent, ranging from $35^{\prime \prime}$ to $43^{\prime \prime}$. In the southern arm, the gaps are at slightly smaller radii of $28^{\prime \prime}<r<36^{\prime \prime}$. After these gaps, the bright $24 \mu \mathrm{m}$ spots along the spiral arms are typically azimuthally offset from the $\mathrm{CO}$ emission. The polar cc (Figure 5, middle left) shows that the offset of the hot dust emission associated with the spiral arms starts at radii of $r>50^{\prime \prime}$ (i.e., after the gaps) with an offset of $\sim 30^{\circ}$ that is radially decreasing to $\sim 5^{\circ}$ and rising again for $r>80^{\prime \prime}$ with a decline thereafter. A recent study by Louie et al. (2013) finds qualitatively similar results while the lower resolution (13") study of Foyle et al. (2011) sees no evidence for offsets. This finding can be easily explained by the spottiness of the bright $24 \mu \mathrm{m}$ peaks that are clearly associated with young massive star formation and the enhancement of $24 \mu \mathrm{m}$ emission in the molecular spiral arms, which become mixed at the $13^{\prime \prime}$ resolution that no longer resolves the spiral arms. It is interesting to note that the bright $24 \mu \mathrm{m}$ spots also do not fall on a straight line, suggesting a certain stochasticity when the initial star formation event occurs with respect to the spiral arm passage.

A low-level component of the $24 \mu \mathrm{m}$ emission coincides with the $\mathrm{CO}$ emission in the spiral arms and extends into the downstream interarm region (Figures 2 (middle row) and 4 (middle left)). The factor 2 times enhancement in the smooth component of the $24 \mu \mathrm{m}$ emission in the arm compared with the interarm region is similar to the average arm/interarm difference in $\mathrm{CO}$ brightness. This suggests that the enhancement simply reflects a constant dust-to-gas ratio. Therefore, the $24 \mu \mathrm{m}$ emission detected in the (molecular) arms is likely not tracing any deeply embedded star formation. Detailed modeling of the dust spectral energy distribution at an angular resolution sufficient to exclude the influence of the bright star forming regions associated with the arms would be required to firmly test this interpretation.

There appears to be only a very broad correlation between the hot dust emission traced by $24 \mu \mathrm{m}$ and the $\mathrm{CO}$ emission when considering the entire PAWS FoV (Figure 6, uppermiddle row). However, comparison of the three individual environments provides more insights. The central disk shows a very steep linear relation, although with significant scatter, while there might be an additional loose relation with a significantly shallower slope present in the spiral arms; the interarm region is almost consistent with no relation at all. The second shallower relation is arising from the low-level $24 \mu \mathrm{m}$ emission seen in the two-dimensional and polar representations (Figures 2 (middle left) and 4 (middle left)).

\subsection{4. (Non-thermal) Radio Continuum}

The RC emission at 6 and $20 \mathrm{~cm}$ in M51 is dominated by nonthermal synchrotron emission (Dumas et al. 2011). Surprisingly, the correspondence to the $\mathrm{CO}(1-0)$ distribution is significantly better than for the hot dust emission (Figure 2, bottom row). While bright RC emission peaks exist along the spiral arms, the RC spiral arms coincide with the molecular gas spiral arms. However, the RC arms appear to be more contiguous and diffuse compared with the $\mathrm{CO}(1-0)$ emission at comparable angular resolution. A wavelet decomposition of the RC suggests that the emission arising from the spiral arms is more diffuse and not composed of many individual point sources (Dumas et al. 2011), confirming the visual impression of Figure 2 (bottom row).

In the polar coordinate system, the brightest knots in the 6 and $20 \mathrm{~cm}$ RC along the spiral arms (Figure 4 (middle right); only shows $20 \mathrm{~cm}$ ) are often offset from the gas arm and align with the brightest regions seen, e.g., in the hot dust emission traced by the $24 \mu \mathrm{m}$ map (Figure 4 , middle right). This suggests that recent star formation is dominating the radio excitation here while another process must cause the enhancement of the RC in the spiral arms. This behavior is only mildly reflected in the polar cc with the $\mathrm{CO}(1-0)$ emission (Figure 5, middle right), where the azimuthal offset is consistent with zero at most radii, except for the center where the radio jet is present and along the spiral arms for $57^{\prime \prime} \leqslant r \leqslant 80^{\prime \prime}$. Interestingly, the azimuthal offset is only $\Phi \sim 8^{\circ}$ (compared with $\Phi \sim 30^{\circ}$ seen for the hot dust emission). The southern arm exhibits a bubble-like feature in the $20 \mathrm{~cm}$ continuum map in the polar coordinate system at a radius of $r \sim 65^{\prime \prime}$ between an azimuthal angle of $220<\Phi<240^{\circ}$.

The inner disk is dominated by the well-known radio jet (e.g., Crane \& van der Hulst 1992; Maddox et al. 2007) emanating from the central active galactic nucleus (AGN), while the molecular ring has an $\mathrm{RC}$ counterpart. Interarm $\mathrm{RC}$ emission is detected, but at a significantly lower level. At $6 \mathrm{~cm}$, a second pair of spiral arms appears mid-way between the prominent molecular arms. New EVLA data taken at $20 \mathrm{~cm}$ show this feature at that wavelength as well (J. Ott, 2013, private communication). The location of this second pair of arms is roughly consistent with the location of "magnetic arms" in the interarm region shown by Fletcher et al. (2011). Already, Tilanus et al. (1988) noted that non-thermal radio emission associated with the spiral arms (after the removal of a "base" disk in their $8^{\prime \prime}$ resolution 6 and $20 \mathrm{~cm}$ short spacing corrected VLA data) coincides with the dust lanes rather than star forming giant $\mathrm{H}$ II complexes probed by $\mathrm{H} \alpha$ imaging.

Two tentative correlations between the RC at 6 and $20 \mathrm{~cm}$ and the $\mathrm{CO}$ emission are apparent in the pixel-by-pixel comparison (Figure 6, two bottom rows): one steeper (present in the center region) and a more shallow one (in the arm region), which separate better in the $20 \mathrm{~cm}$ continuum versus $\mathrm{CO}$ emission plot. The situation in the interarm regions seems to be more complex, judging from the significantly larger scatter in the respective diagrams. The steeper correlation is also significantly stronger in the central disk while the shallower relation dominates in the spiral arms and (tentatively) the interarm region. It is interesting to note that in the spiral arm region of the $6 \mathrm{~cm}$ versus $C O$ emission (Figure 6, middle-right panel in the second to last row), there is an indication for a down turn at large CO fluxes, which might point toward synchrotron losses in very dense gas. Better data are required to confirm this tentative trend. Slopes between the $\mathrm{CO}$ flux and the $6 \mathrm{~cm}$ as well as the $20 \mathrm{~cm} \mathrm{RC}$ density are not identical. The few points scattered along an almost vertical branch (especially evident in the center diagram of the $6 \mathrm{~cm}$ emission) are likely caused by the prominent radio jet.

\subsubsection{PAH Emission}

Our stellar continuum corrected IRAC images at $3.6 \mu \mathrm{m}$ and $8 \mu \mathrm{m}$ contain two non-stellar emission components, namely PAH emission features and hot dust continuum. The $3.6 \mu \mathrm{m}$ image covers the $3.3 \mu \mathrm{m}$ PAH feature while the $8 \mu \mathrm{m}$ image 

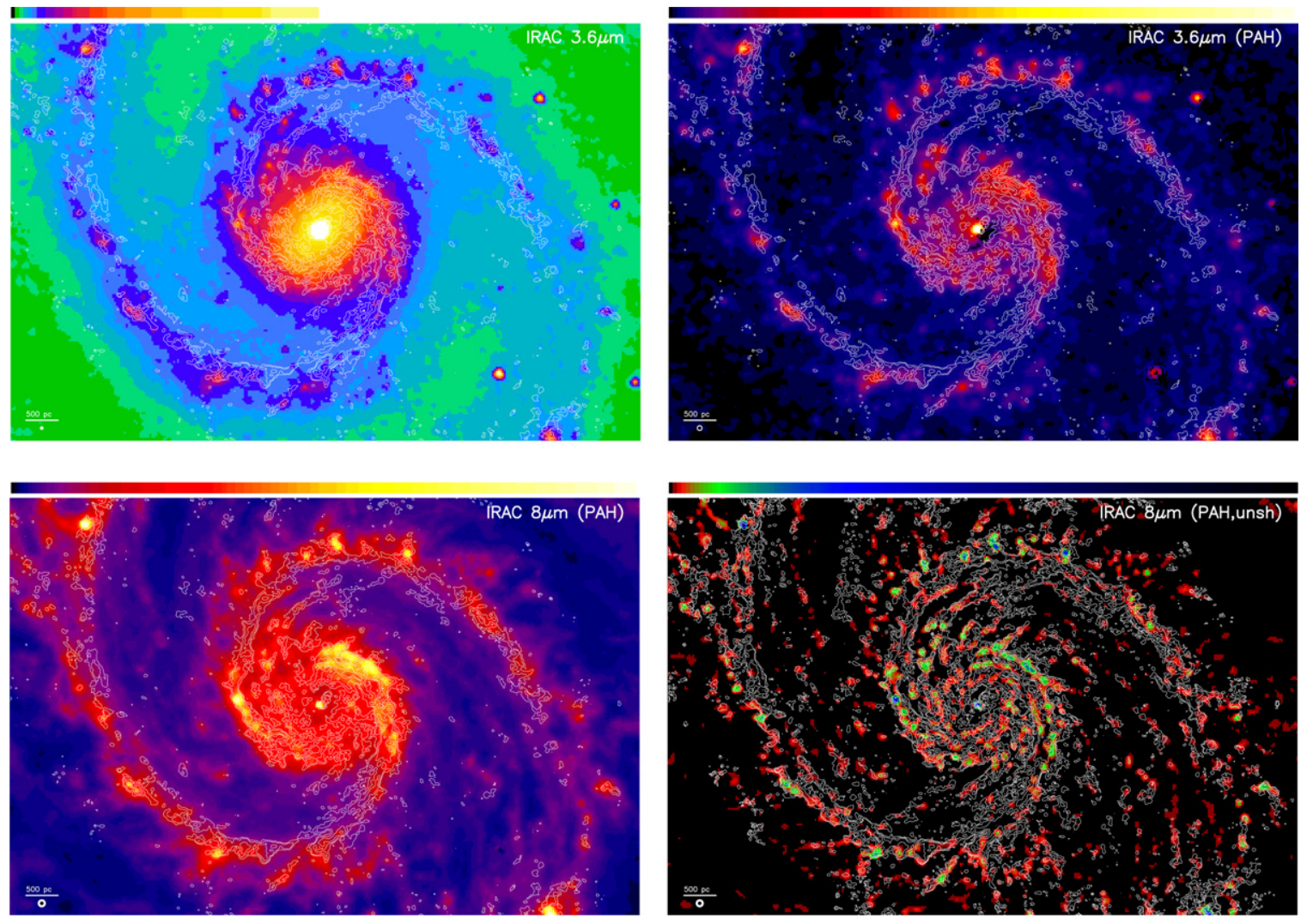

Figure 3. Comparison of the molecular gas distribution of M51a with tracers of $\mathrm{PAH}$ emission. ${ }^{12} \mathrm{CO}(1-0)$ intensity distribution overlaid onto the total $3.6 \mu \mathrm{m}$ emission (top left) and its non-stellar component only (top right), the $8 \mu \mathrm{m}$ non-stellar component mainly representing PAH emission (bottom left), and its unsharped masked version (bottom right), where an extra ${ }^{12} \mathrm{CO}(1-0)$ contour at $20 \mathrm{~K} \mathrm{~km} \mathrm{~s}^{-1}$ is added. In the bottom-left corner of each panel, a scale bar representing $500 \mathrm{pc}$ and the CLEAN beam of the $\mathrm{CO}(1-0)$ data, as well as the resolution of the respective data set, are shown.

captures emission from the prominent PAH features at 7.7 and $8.6 \mu \mathrm{m}$. Comparison of the stellar continuum corrected $8 \mu \mathrm{m}$ (Figure 3, bottom left) and $3.6 \mu \mathrm{m}$ images (Figure 3, top right) with the distribution of the $\mathrm{CO}$ emission shows that the nonstellar 3.6 and $8 \mu \mathrm{m}$ emission is cospatial with the $\mathrm{CO}$ emission in the ring and at smaller radii. Neglecting the nucleus (where the AGN could contribute to or even dominate the emission seen), very bright $3.6(8) \mu \mathrm{m}$ non-stellar emission is found in the northwestern part of the ring close to the brightest $\mathrm{CO}$ emission in the ring. As this is along the direction of the radio jet, it is not clear if this is just a coincidence.

The spatial coincidence between non-stellar 3.6 (8) $\mu \mathrm{m}$ and $\mathrm{CO}$ emission becomes less perfect along the spiral arms. The brightest emission regions at 3.6 (8) $\mu \mathrm{m}$ are just situated outside the molecular spiral arms. However, roughly one order of magnitude fainter non-stellar $3.6 \mu \mathrm{m}$ emission $\left(\sim 0.15 \mathrm{MJy} \mathrm{sr}^{-1}\right)$ is associated with a significant portion of the molecular spiral arms. The fainter $8 \mu \mathrm{m}$ PAH emission in the interarm region exhibits a filamentary structure connecting the two spiral arms. We find that our CO emission aligns well with the brighter regions, especially when looking at an unsharped version of the $8 \mu \mathrm{m}$ PAH image (Figure 3, bottom right). The non-detection of $\mathrm{CO}$ emission from the faintest filaments in the interarm region might thus be due to our limited CO sensitivity. Overall, the spatial distribution of the PAH emission seems to be a very good predictor of the $\mathrm{CO}$ emission while deviations are seen in regions of massive star formation.

The high surface brightness peaks seen in both the non-stellar 3.6 and $8 \mu \mathrm{m}$ emission maps along the spiral arm (Figure 4, bottom left and right) correlate very well with the peaks present in the $24 \mu \mathrm{m}$ map (Figure 4, middle left), indicating that hot dust could contribute in addition to the PAH features. The very similar radial profile of the polar cc of the non-stellar $3.6 \mu \mathrm{m}$ emission with the $\mathrm{CO}$ emission (Figure 5, bottom left) supports this notion. The cross-correlation profile of the non-stellar $8 \mu \mathrm{m}$ emission (Figure 5, bottom right) exhibits a different shape with an azimuthal offset typically smaller than the one seen in the hot dust emission (see Section 4.2.3). The average offset is only $\Phi \sim 15^{\circ}$ and shows much less pronounced variations with radius in the spiral arm region of $r>55^{\prime \prime}$. The difference in the cc profiles of the non-stellar 3.6 and $8 \mu \mathrm{m}$ emission can be explained with different amounts of hot dust contribution. The distribution of the faint PAH emission is more obvious in the $8 \mu \mathrm{m}$ PAH emission due to better sensitivity (Figure 4, bottom right). The faint PAH emission follows the gas spiral arms and the $\mathrm{CO}$ distribution in the ring very well. The gaps seen at $3.6 \mu \mathrm{m}$ in the spiral arms at roughly $r \approx 35^{\prime \prime}$ are not obvious at $8 \mu \mathrm{m}$, suggesting that the gaps are due to a lack of sensitivity and not necessarily a lack of PAH emission. It is interesting to note that the spiral arms are wider in the nonstellar $8 \mu \mathrm{m}$ emission than seen in $\mathrm{CO}$, especially in the radial range where prominent star formation is present.

Both non-stellar components at 3.6 and $8 \mu \mathrm{m}$ have two broad relations with $\mathrm{CO}$ emission with slightly differing slopes and a prominent vertical offset in PAH intensity (Figure 7, top two rows). However, there is no obvious dividing line between these two relations. As shown by Meidt et al. (2012), the $3.6 \mu \mathrm{m}$ non-stellar emission can be a combination of PAH features and 

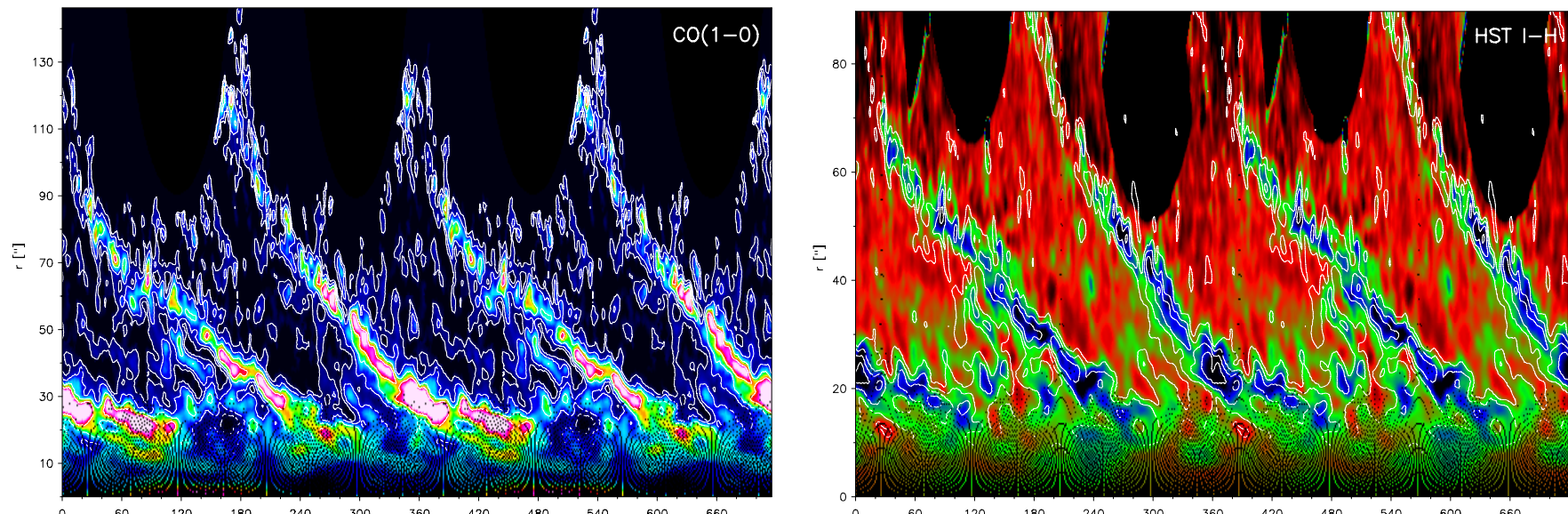

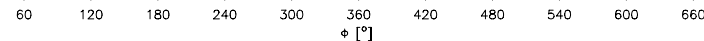
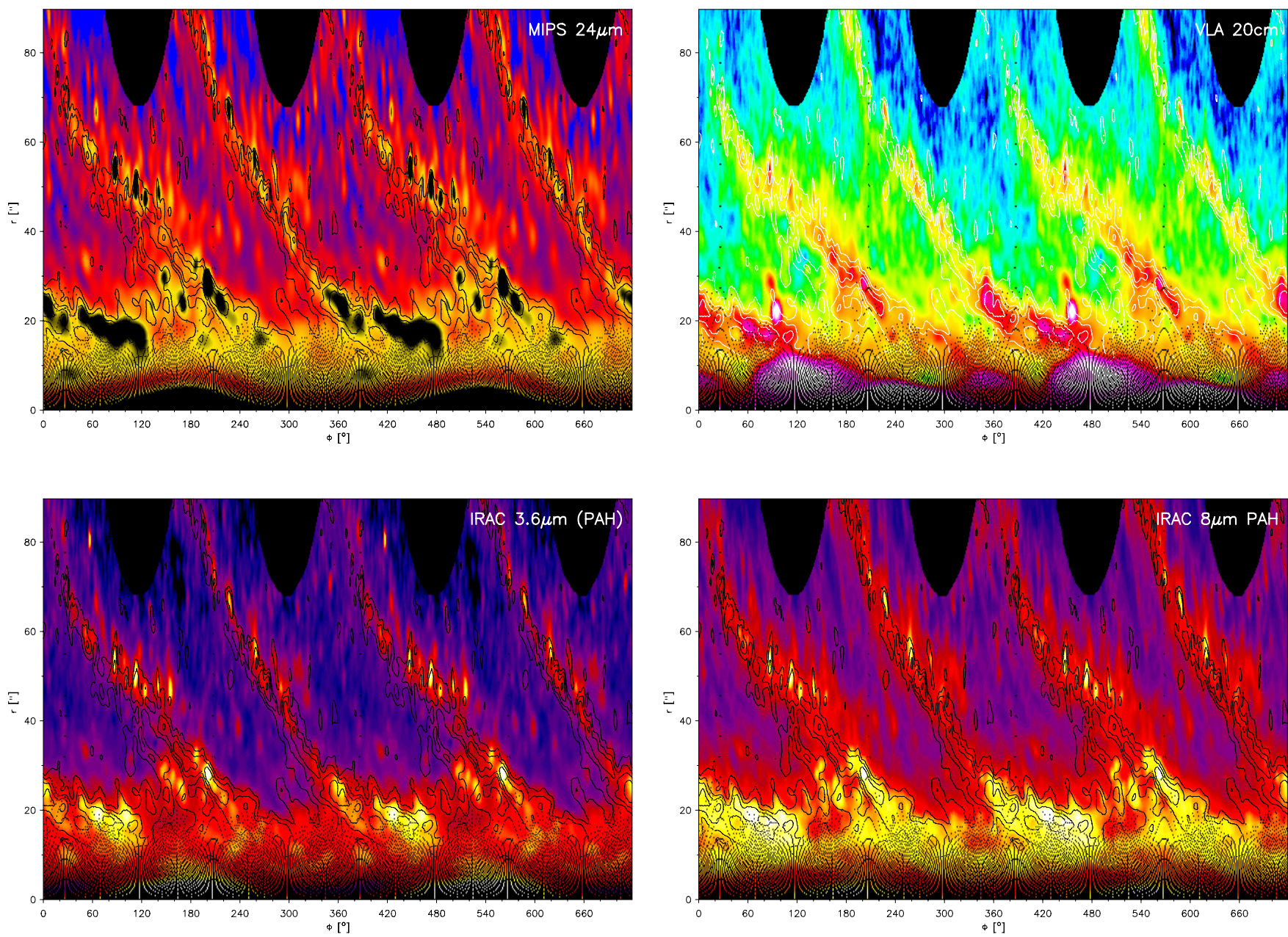

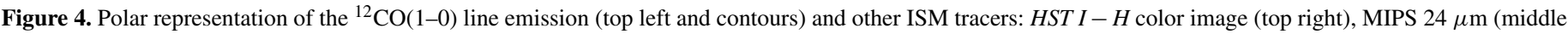
left), VLA $20 \mathrm{~cm}$ (middle right), and the non-stellar continuum at IRAC $3.6 \mu \mathrm{m}$ and IRAC $8 \mu \mathrm{m}$ (bottom).

hot dust, especially in regions of active massive star formation, thus a single relation is not necessarily expected. As the upper relation is most prominent in the central region (Figure 7, middle left), this strongly suggests that a contribution from hot dust is at least adding to this relation. The ratio of $24 \mu \mathrm{m}$ and non-stellar $8 \mu \mathrm{m}$ emission (Figure 7, lower-middle row) also exhibits a steeper slope in the center, consistent with enhanced hot dust contribution to the non-stellar $8 \mu \mathrm{m}$ light. Basically, no relation between the ratio of $24 \mu \mathrm{m}$ and non-stellar $8 \mu \mathrm{m}$ and the $\mathrm{CO}$ emission is seen for the spiral arms, suggesting that this might be indeed genuine $\mathrm{PAH}$ emission that correlates with dense molecular gas. As the bulk of points in the interarm region correspond to higher PAH intensities than in the arm region, we speculate that both regimes-hot dust mixed with PAHs as well as PAHs only-are present, although on a significantly lower level than observed for the center. The ratio of the 


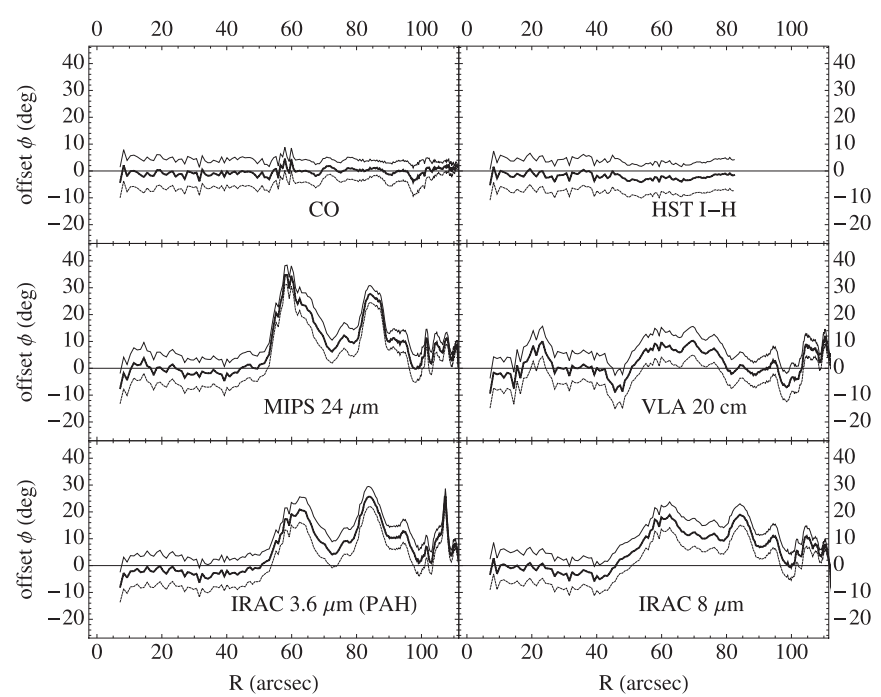

Figure 5. Results of the polar cc between the ${ }^{12} \mathrm{CO}(1-0)$ line emission (top left) and other ISM tracers. The panels show the radial profiles of the location of maximum correlation between the ${ }^{12} \mathrm{CO}(1-0)$ intensity and the emission from other tracers, tracing the azimuthal offset $\Phi$ between the two: ${ }^{12} \mathrm{CO}(1-0)$ intensity (top left), $H S T I-H$ image (top right), MIPS $24 \mu \mathrm{m}$ image (middle left), VLA $20 \mathrm{~cm}$ continuum (middle right), and non-stellar continuum at IRAC $3.6 \mu \mathrm{m}$ and $8 \mu \mathrm{m}$ (bottom). The thin lines represent the uncertainty defined as the width of the cc profile at $95 \%$ maximum correlation.

non-stellar $8 \mu \mathrm{m} / 3.6 \mu \mathrm{m}$ emission shows some dependence on $\mathrm{CO}$ emission as a function of galactic environment. The smaller scatter of this relation in the central disk could be interpreted as being dominated by a (constant) hot dust component, while in the spiral arms the scatter in this ratio becomes large, reflecting the large range of physical conditions sampled in this region. The larger scatter implies either a more varying hot dust contribution or an intrinsic variation of the ratio of the PAH features sampled by the $3.6 \mu \mathrm{m}$ and $8 \mu \mathrm{m}$ filters.

\subsection{Comparison of $\mathrm{CO}(1-0)$ with Star Formation and Stellar Tracers}

Comparison of the morphology and distribution of the molecular gas that is viewed as the fuel for star formation (e.g., Bigiel et al. 2008, 2011; Leroy et al. 2008; Schruba et al. 2011) with tracers sensitive to star formation with varying age sensitivities is illustrative to study the relation between the fuel and the endproduct on cloud scales. The $\mathrm{H} \alpha$ emission from $\mathrm{H}$ II regions traces the youngest star forming sites between a few and about 10 Myr (e.g., Whitmore et al. 2011). The thermal RC should trace the same population of stars responsible for the ionization of the HII regions, however it is basically unaffected by the presence of dust (Condon 1992; Murphy et al. 2011, 2012). The $B$ - and $V$-band light from young stellar clusters probes ages out to several tens of Myr (for results on M51, see Chandar et al. 2011; Bastian et al. 2005; Scheepmaker et al. 2007, 2009; Haas et al. 2008; Hwang \& Lee 2008). Likewise, the FUV and NUV emission can arise from main sequence stars as late as A type, again being sensitive to ages of up to $\sim 100$ Myr. Finally, the $H$-band and $3.6 \mu \mathrm{m}$ filters are most sensitive to a wavelength range where an old stellar population consisting of red giants dominates the spectral energy distribution (in the absence of red supergiants and/or asymptotic giant branch stars, which have typical lifetimes of a few 10 to $10^{3} \mathrm{Myr}$ and should also exhibit a clustered distribution).

\subsubsection{H II Regions}

Most of the $\mathrm{H} \alpha$ emission is closely associated with the molecular gas (Figure 8, top left). In the center, the $\mathrm{H} \alpha$ emission coincides with the $\mathrm{CO}$ emission out to a radius of $r \sim 35^{\prime \prime}$ (Figure 10, top left). A partial gap is present adjacent to the inner gas disk and continues out to a radius of $r \sim 41^{\prime \prime}$. After this gap, the $\mathrm{H} \alpha$ emission tends to be offset toward the convex side of the $\mathrm{CO}$ spiral arm by about $(9-18)^{\circ}$ at a given radius. It is interesting to note that the $\mathrm{H} \alpha$ emission is not forming a straight line but is oscillating randomly as a function of radius within this offset range. ${ }^{17}$ Particularly, the $\mathrm{H} \alpha$ morphology along the spiral arms exhibits large diffuse envelopes and shells, signaling the presence of giant HiI regions (see also Lee et al. 2011; Gutiérrez et al. 2011). These very bright and large H II regions tend to overlap with the gas spurs emanating from the gas spiral arms. Faint $\mathrm{H} \alpha$ emission is also detected in the interarm region with an apparent preference for being mid-way between the two spiral arms.

There is no obvious relation between the $\mathrm{H} \alpha$ emission related to $\mathrm{H}$ II regions and the $\mathrm{CO}$ emission when comparing it on a pixel-by-pixel basis in the full PAWS FoV (Figure 12, top row). A closer inspection of the individual region shows that $\mathrm{H} \alpha$ emission in the central disk exhibits some complex relation, while we see basically no relation between the $\mathrm{H} \alpha$ and $\mathrm{CO}$ line emission in the arm and interarm regions.

\subsection{2. (Thermal) Radio Continuum}

Unlike the RC at longer wavelengths (see Section 4.2), the $3.6 \mathrm{~cm} \mathrm{RC}$ is distributed in a more patchy fashion and dominated by bright emission peaks (Figure 8 , top right). This suggests that the thermal contribution in these regions is higher. The radio jet is still the brightest feature seen in the central part. The continuum emission is cospatial with the CO emission in the central disk. Diffuse, faint $3.6 \mathrm{~cm}$ radio emission is associated with the gas spiral arms (Figure 10, top right), while the bright peaks along the spiral arms show a similar offset from the $\mathrm{CO}$ emission as the $\mathrm{H} \alpha$ emission. About half of these peaks can be directly related to the brightest $\mathrm{H}$ II regions. The polar cc with the $\mathrm{CO}$ emission (Figure 11, top left) shows a very similar radial profile to the one seen for the $20 \mathrm{~cm}$ continuum, with a similar azimuthal offset of $\sim 10^{\circ}$. The lack of strong $3.6 \mathrm{~cm}$ emission within the CO spiral arms implies that no strong star formation is hidden in the molecular gas spiral arms.

It is interesting to note that the non-thermal/thermal separation of their $8^{\prime \prime}$ resolution 6 and $20 \mathrm{~cm}$ radio imaging led Tilanus et al. (1988) to conclude that the thermal emission solely arises from giant $\mathrm{H}$ II complexes without any evidence for such giant H II complexes being hidden in the dust lanes. They did not rule out the existence of significantly smaller $\mathrm{H}$ II regions based on the sensitivity achieved in their radio maps. Given the fact that the radio imaging used here has an rms 2.5 times and 11 times lower while achieving 16 times and 35 times higher spatial resolution (in beam area) at 6 and $20 \mathrm{~cm}$, respectively, and that no prominent bright peaks have appeared at our resolution inside the spiral arms suggests that not much hidden massive star formation is occurring inside the gas spiral arms.

Similar to the 6 and $20 \mathrm{~cm} \mathrm{RC}$ emission, the $3.6 \mathrm{~cm} \mathrm{RC}$ shows three broad relations with the $\mathrm{CO}$ emission in the pixelby-pixel diagrams (Figure 12, second row from the top): (1) a large range in radio flux density for a fixed low CO intensity, 17 The application of the polar cc was not successful due to the patchiness of
the $\mathrm{H} \alpha$ emission. 



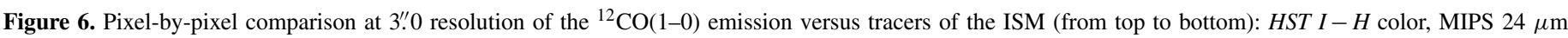

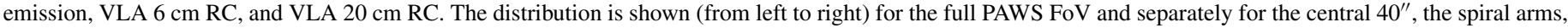
and the interarm region (as defined in Figure 1). The density is given in a logarithmic color scale.

(2) a steep relation, and (3) a possibly very shallow or even no relation between (low) $3.6 \mathrm{~cm}$ flux density and $\mathrm{CO}$ intensity. The explanation is analogous to the 6 and $20 \mathrm{~cm} \mathrm{RC:} \mathrm{the} \mathrm{radio} \mathrm{jet} \mathrm{is}$ causing the large range of radio flux for a given $\mathrm{CO}$ intensity in the center, while the steeper relation that is mainly present in the center is related to the larger amount of star formation whereas the very shallow one is exclusively seen in the arm and interarm regions and can be interpreted as non-thermal emission (see the discussion in Section 5.2).

\subsubsection{Young Stellar Clusters and Stars}

The presence and location of young stellar clusters relative to the molecular gas is best seen in the HST B and $V$ bands (Figure 8, middle panels). The stellar light is significantly stronger in the center, reflecting the presence of a stellar bulge. Prominent dust lanes are seen in both the $B$ - and $V$-band images tracing the spiral arms as well as an intricate web in the center. As the CO emission follows these dust lanes very well, a clear anticorrelation with the distribution of young stellar clusters is seen (Figure 10, middle left). As the correspondence of CO emission to dust lanes is also very good in the center, dust lanes appear to be a superb predictor of the location of $\mathrm{CO}$ emission.

The $B$-band emission and $\mathrm{CO}$ spiral arms appear to converge and meet at a radius of $r \sim 78^{\prime \prime}$. There is a gap in the $B$-band emission at roughly $32^{\prime \prime}<r<42^{\prime \prime}$ that is more pronounced along the southern arm while along the northern arm a few small stellar clusters are present. The distribution of stellar clusters is significantly enhanced along the convex sides of both gas spiral arms. The distribution is wider and brighter along the southern spiral arm while more clustering of stellar clusters is seen along the northern spiral arm. Overall, the width of the arms as seen in the (young) stellar clusters appears significantly wider than the $\mathrm{CO}$ arms. Similar to the $\mathrm{H} \alpha$ emission, there are a few prominent strings of stellar clusters mid-way between the spiral arms in the interarm regions.

The azimuthal offset between the bright $\mathrm{CO}$ and $B$-band emission is larger beyond a radius of $r \approx 55^{\prime \prime}$ and decreases until a radius of $r \approx 80^{\prime \prime}$. (The negative offset for radii $r \leqslant 45^{\prime \prime}$ is similar to that seen for the old stellar component and discussed there; see Section 4.3.4.) The $V$-band emission (Figure 10, middle right) shows a very similar picture to the $B$ band and has also gaps. It is interesting to note that the $V$-band emission is basically anti-correlated with the $24 \mu \mathrm{m}$ emission along the northern spiral arm. The overall brightness in the $V$ (and $B$ ) band falls rapidly out to a radius of $r \sim 32^{\prime \prime}$ before leveling out to the disk value. The polar cc analysis of the $V$-band continuum with the $\mathrm{CO}$ emission (Figure 11, top right) reveals a negative azimuthal offset for radii of $r<42^{\prime \prime}$ that becomes a positive azimuthal offset beyond $r>50^{\prime \prime}$, showing a similar radial dependence as the hot dust emission (Figure 5, middle left), 

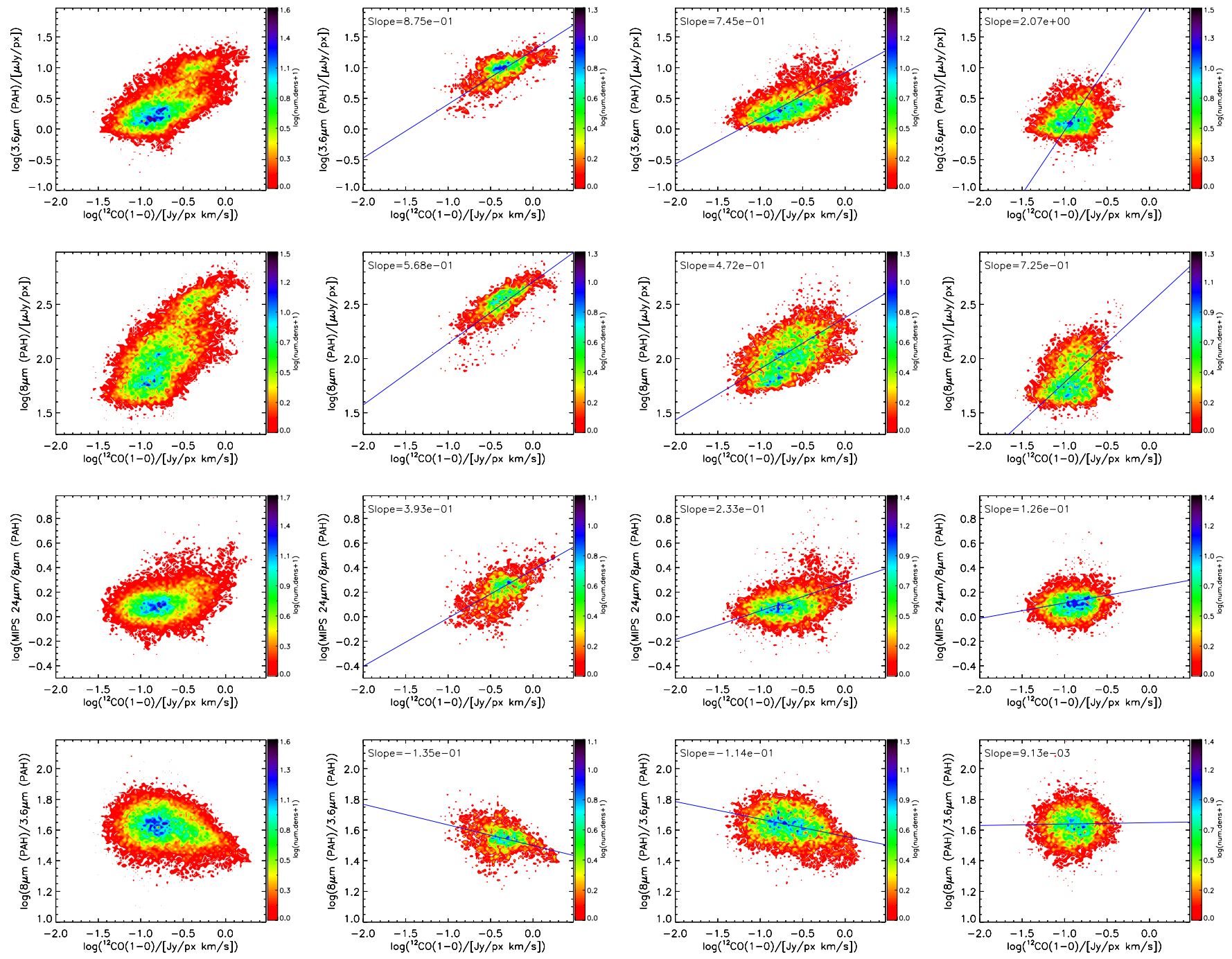

Figure 7. Pixel-by-pixel comparison at 3."0 resolution of the ${ }^{12} \mathrm{CO}(1-0)$ emission versus tracers of the PAH emission (from top to bottom): non-stellar emission at $3.6 \mu \mathrm{m}$, non-stellar emission at $8 \mu \mathrm{m}$, the ratio of $24 \mu \mathrm{m} / 8 \mu \mathrm{m}_{\text {non-stellar }}$, and the ratio of non-stellar emission at $8 \mu \mathrm{m}$ over $3.6 \mu \mathrm{m}$. The distribution is shown (from left to right) for the full PAWS FoV and separately for the central $40^{\prime \prime}$, the spiral arms, and the interarm region (as defined in Figure 1). The density is given in a logarithmic color scale.

however, with a much less pronounced amplitude, i.e., a smaller azimuthal offset.

The UV continuum arises from stars with ages less than $\sim 100 \mathrm{Myr}$ and is thus a good proxy for recent star formation. The GALEX FUV and NUV images (Figure 8, bottom panels) exhibit a bright, slightly patchy central disk with $\mathrm{CO}$ emission often falling into regions of less bright UV emission. The extent of the central UV disk is larger than the $\mathrm{CO}$ disk. In addition, two prominent spiral arms are present. Interestingly, these UV spiral arms do not connect to the central disk but show a broad gap. The UV light along the spiral arms is offset toward the convex side of the spiral arms seen in $\mathrm{CO}$ emission and shows several emission peaks that are embedded in a fainter smooth component that is best visible in the NUV continuum along the southern arm.

As is the case for the $\mathrm{H}$ II regions, there is no obvious relation between the emission from young stellar populations (using the HST B-band as a proxy) and the $\mathrm{CO}$ emission (Figure 12, middle row). The large range in $B$-band fluxes in the pixel-bypixel diagram for the center reflects the presence of the stellar bulge. As the emission from the stellar disk and individual stellar clusters is more homogeneous in intensity, the range is much lower in the arm and interarm regions. It is interesting to note that the anti-correlation seen in the images is reflected in the negative relation between $H S T B$-band flux density and $\mathrm{CO}$ flux present in the arm region, i.e., for a higher $\mathrm{CO}$ flux, less $B$-band light is observed.

\subsubsection{Old Stellar Population}

The old stellar population should be a good proxy for the underlying stellar potential and thus the possible driving force of the observed $\mathrm{CO}$ distribution. The HST $\mathrm{H}$-band image exhibits a bright bulge that has roughly the extent of the molecular disk (Figure 9, left). Bright stellar clusters are plentiful along the spiral arms, similar to their distribution in the HST B- and $V$-band images (Figure 9, middle panels). Given the possibly large contribution from younger stellar clusters, the contaminant-corrected $3.6 \mu \mathrm{m}$ map of Meidt et al. (2012) provides a better view of the old stellar population alone and its spatial relation to the molecular gas (Figure 9, right). The nuclear bar (e.g., Zaritsky et al. 1993) is easily identified and sits inside the molecular gas ring. The molecular gas ring, or more precisely the tightly wound $\mathrm{CO}$ spiral arms, appears to start at 

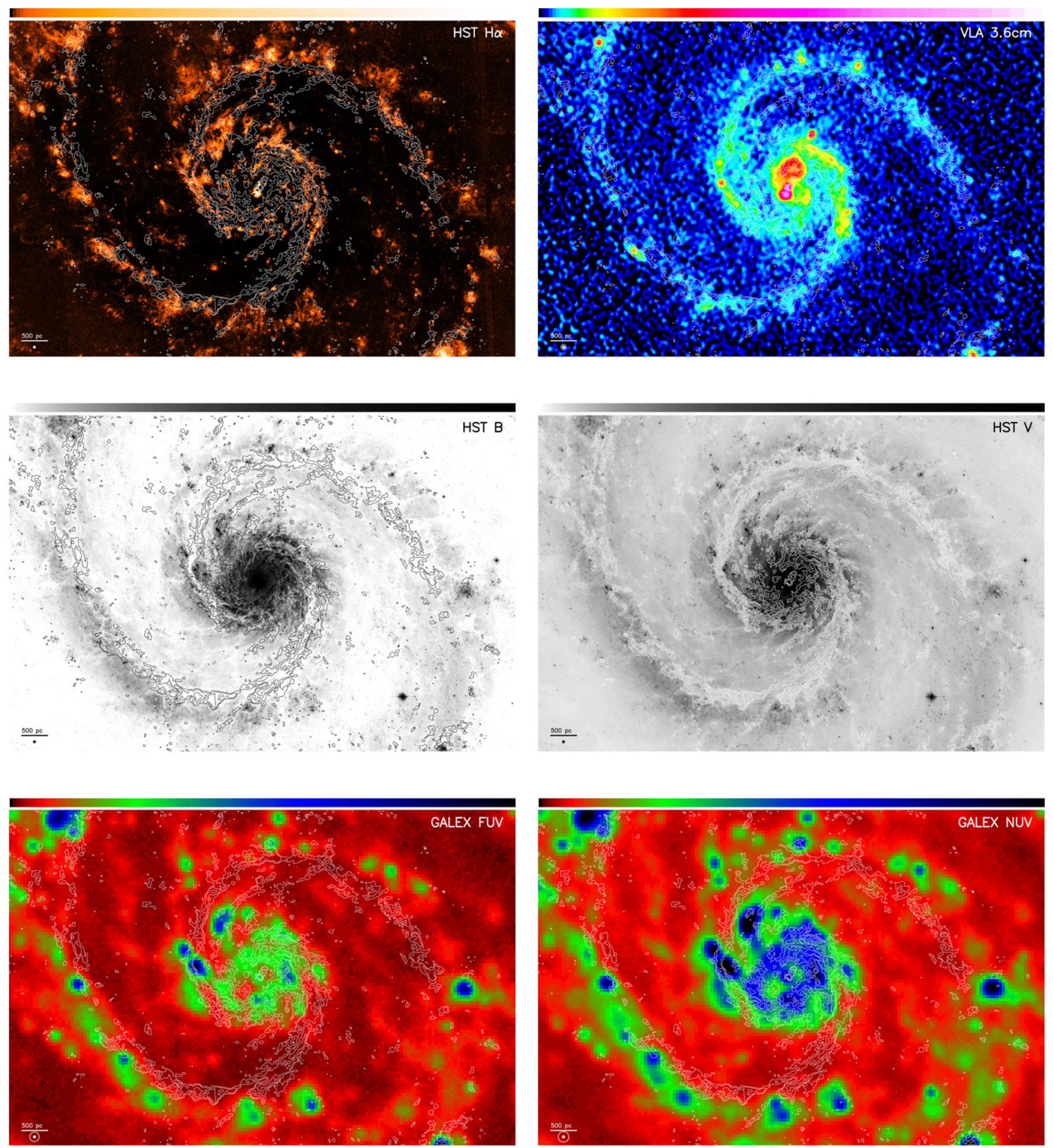

Figure 8. Comparison of the $\mathrm{CO}(1-0)$ line emission of M51a with SFR tracers. ${ }^{12} \mathrm{CO}(1-0)$ intensity distribution with contours overlaid onto the $H S T$ H $\alpha$ line emission showing prominent $\mathrm{H}$ II regions (top left), the VLA $3.6 \mathrm{~cm}$ image tracing thermal and non-thermal RC (top right), the $H S T B$-band (middle left) and $V$-band (middle right) image tracing young clusters, and the GALEX FUV (bottom left) and NUV (bottom right) image tracing UV emission from young ( $\leqslant 100 \mathrm{Myr})$ stars. In the bottom-left corner of each panel, a scale bar representing $500 \mathrm{pc}$ and the CLEAN beam of the $\mathrm{CO}(1-0)$ data, as well as the resolution of the respective data set, are shown.

its tips. Both spiral arms are also visible in this image, implying that the gravitational potential should have at least one $m=2$ mode where the molecular gas spiral arms are. The southern spiral arm is about $10 \%-20 \%$ brighter than the northern arm. Assuming a constant mass-to-light ratio, this would imply that the southern arm is about 1.1-1.2 times more massive than the northern arm. The peaks evident in the contaminant-corrected
$3.6 \mu \mathrm{m}$ image are likely artifacts due to imperfect correction in the regions of most intense star formation (Meidt et al. 2012).

In the polar representation, a slight dip in $H$-band surface intensity is evident at the location of the $\mathrm{CO}$ spiral arm (Figure 10, bottom left). ${ }^{18}$ This suggests that even the $H$-band

\footnotetext{
18 The black points are artifacts caused by hot pixels.
} 

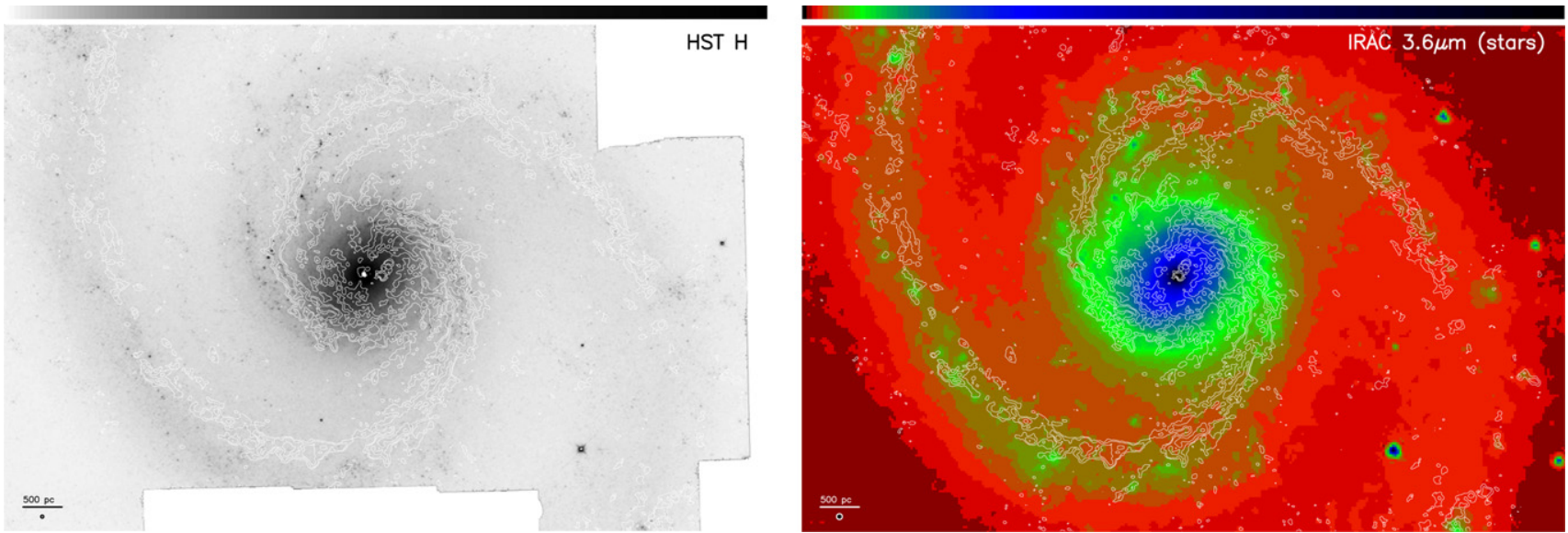

Figure 9. Comparison of the $\mathrm{CO}(1-0)$ distribution with the old stellar populations, as seen in the HST NICMOS $H$-band image (left) and the contamination-corrected stellar light at $3.6 \mu \mathrm{m}$ (right, from Meidt et al. 2012) that represents the underlying gravitational potential. In the bottom-left corner of each panel, a scale bar representing $500 \mathrm{pc}$ and the CLEAN beam of the $\mathrm{CO}(1-0)$ data, as well as the resolution of the respective data set, are shown.
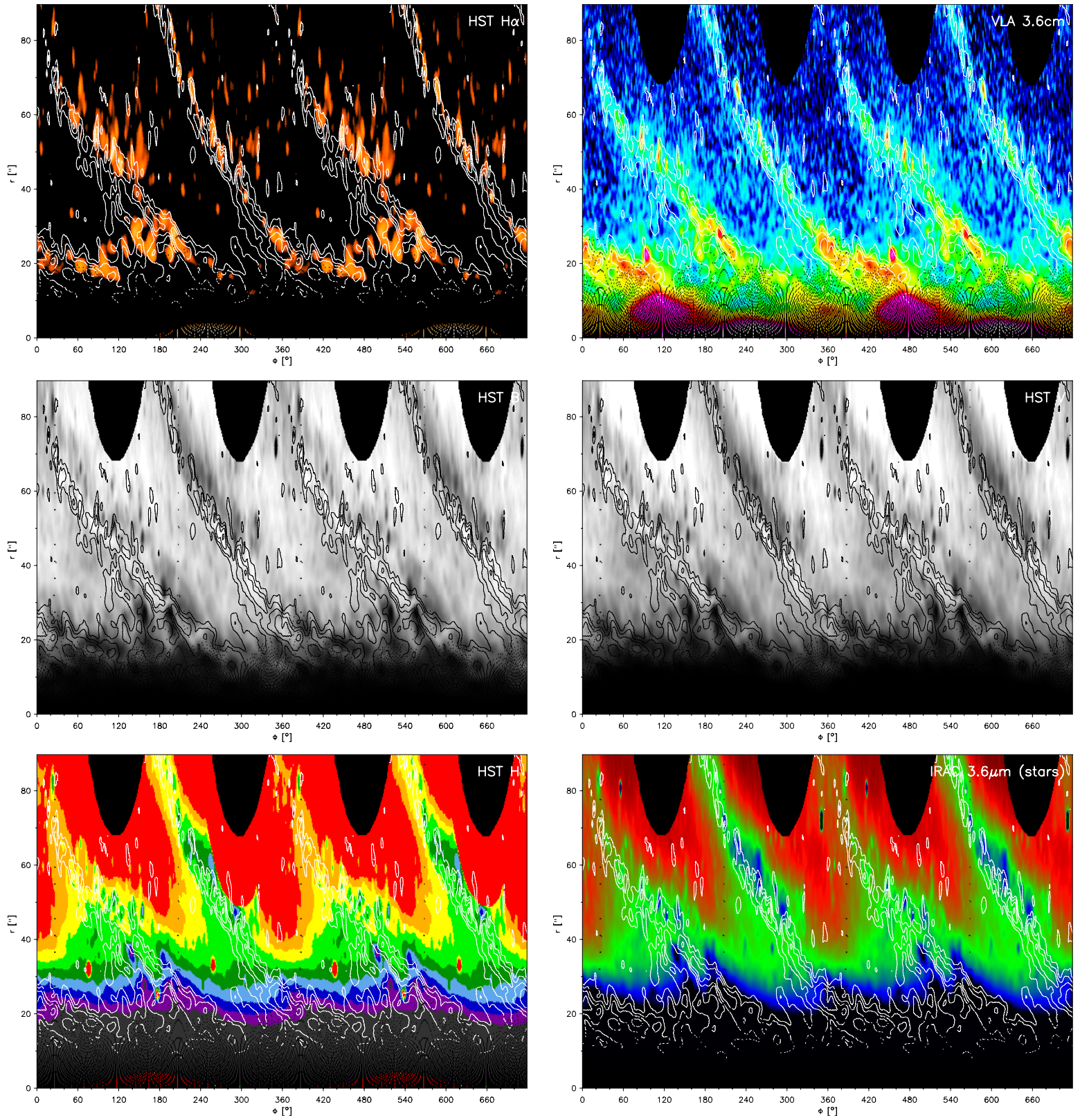

Figure 10. Polar representation of the ${ }^{12} \mathrm{CO}(1-0)$ line emission (contours) overlaid onto SFR and stellar population tracers: HST H $\alpha$ image (top left), VLA $3.6 \mathrm{~cm}$ (top right), HST B- and $V$-band images (middle), HST $H$-band image (bottom left), and the contaminant-corrected IRAC $3.6 \mu \mathrm{m}$ image (bottom right). 




Figure 11. Results of the polar cc between the ${ }^{12} \mathrm{CO}(1-0)$ line emission and SFR and stellar population tracers. The panels show the radial profiles of the location of maximum correlation between the ${ }^{12} \mathrm{CO}(1-0)$ intensity and the emission from the other tracers, tracing the azimuthal offset $\Phi$ between the two: VLA $3.6 \mathrm{~cm}$ continuum (top left), HST $B$-band image (top right), $H S T H$-band image (bottom left), and the contaminant-corrected IRAC $3.6 \mu \mathrm{m}$ image (bottom right). The thin lines represent the uncertainty defined as the width of the cc profile at $95 \%$ maximum correlation.

light is affected by extinction there. The contaminant-corrected $3.6 \mu \mathrm{m}$ image (Figure 10, bottom right) exhibits a sinusoidal profile at small radii that reflects the geometry of the inner bar out to a radius of $r \sim 17^{\prime \prime}$ at an angle of, e.g., $\theta \sim 185^{\circ}$, corresponding to a PA (measured north through east) of $\sim 147^{\circ}$, close to the value of $\mathrm{PA}=139^{\circ}$ for the nuclear bar derived by Menéndez-Delmestre et al. (2007). A second modulation in the radial range of $25^{\prime \prime} \leqslant r \leqslant 50^{\prime \prime}$ is prominent at, e.g., an azimuthal angle of $40^{\circ} \leqslant \theta \leqslant 160^{\circ}$. This modulation has a very wide peak $\left(\theta \sim 100^{\circ}\right)$. The width of this modulation decreases significantly at radii larger than $r \approx 50^{\prime \prime}$. The most likely explanation is that in addition to the spiral arms, which start beyond $r \approx 50^{\prime \prime}$, another component of the galactic potential, like a wide oval, is present. As the width of the modulation at the largest radii probed is significantly smaller, the spiral arms likely affect a small part of the disk at a given radius.

As already seen for the polar cc of the HST B band, both the $H S T H$-band and the non-stellar continuum-corrected $3.6 \mu \mathrm{m}$ emission (Figure 11, bottom panels) exhibit a negative azimuthal offset of $\Phi \sim 5^{\circ}$ compared with the $\mathrm{CO}$ emission for radii of $r<42^{\prime \prime}$. This negative offset can be easily understood as these radii are inside the corotations of the nuclear bar and the spiral arms (plus the potential oval) and therefore it is expected that the gas response is leading the gravitational potential of these nonaxisymmetric components that are most prominent in the old stellar light. Beyond radii of $r>50^{\prime \prime}$, the profile is consistent with no azimuthal offset. We cannot exclude that there is a residual effect from light contributed by the younger stellar population that leads to the almost exact zero offset along the spiral arms as the azimuthal offset decreases from the $B$ band to the stellar $3.6 \mu \mathrm{m}$ to the $H$-band emission.

As expected there is, in general, no correlation between $\mathrm{CO}$ emission and the light of the old stellar population as traced by their $H$-band and contaminant-corrected $3.6 \mu \mathrm{m}$ emission (Figure 12, bottom two rows). Interestingly, the central region shows evidence for a linear relation between $\mathrm{CO}$ flux and old stellar light, however, with a second plume of points that shows no correlation with $\mathrm{CO}$ flux. A possible explanation could be excess emission caused by young(er) stars. This seems unlikely as the light distribution is very smooth in the $H$-band and the contaminant-corrected $3.6 \mu \mathrm{m}$ images (Figure 9). A further argument against contribution from young stars is the pixel- by-pixel diagram of the HST $B$-band light versus $\mathrm{CO}$ emission that shows no correlation at all in the center. Thus, it seems plausible that the stellar bulge contributes to an enhancement of $\mathrm{CO}$ emission, e.g., via heating. On the other hand, no relation between the old stellar population and the $\mathrm{CO}$ emission is detected in the two disk regions (arm, interarm).

\section{THE MOLECULAR ISM ON CLOUD SCALES}

In the following, we place our findings in the context of the different galactic environments that are probed by the PAWS FoV. The high spatial resolution of $40 \mathrm{pc}$ of the PAWS data allows us to better probe the physical origins of the correlations seen among the different ISM and stellar tracers and the CO emission.

Broadly speaking, the energy density fields that affect the ISM can be categorized in radiation fields, cosmic rays (CRs), kinetic energy fields (e.g., large-scale turbulence), and magnetic fields. We discuss only the relation between the distribution of the molecular gas and CRs (Section 5.2), as well as the radiation field (Section 5.3), i.e., from stars and the AGN. The role of the kinetic energy in organizing the molecular gas and the injection of (large-scale) turbulence is discussed in companion papers (Colombo et al. 2013a; Hughes et al. 2013b; Meidt et al. 2013). As no high angular resolution, high sensitivity studies of the magnetic field in M51 exist, we do not investigate the importance of the magnetic field for determining the molecular gas properties. As the gravitational potential basically organizes both the distribution of the ISM and the energy density fields that affect it, we start with a brief description of the different galactic component of the gravitational potential present in the PAWS FoV (Section 5.1). This will provide a guideline when we try to separate the different components of the energy density field(s) that affect the ISM.

\subsection{Gravitational Potential and the ISM Geometry}

The shape of the gravitational potential determines the distribution of both the ISM and the energy density fields and thus the different galactic environments the molecular gas resides in. The old stellar population can be considered the best tracer of the underlying stellar potential (Rix \& Rieke 1993). Based on the distribution of the old stellar component (from Section 4.3.4) and literature results (e.g., Rix \& Rieke 1993; Zaritsky et al. 1993; Beckman et al. 1996; Lamers et al. 2002; Henry et al. 2003; Menéndez-Delmestre et al. 2007), we identify the following components in the PAWS FoV (from the center outward):

1. a central supermassive black hole that is actively accreting (e.g., Maddox et al. 2007, and reference therein)

2. a bulge with a radial extent of $r \leqslant 16^{\prime \prime}$ (Fisher \& Drory 2010), and

3. a galactic disk that contains

(a) an inner nuclear bar with a major axis length of $r \sim(15-17)^{\prime \prime}$ and an orientation of PA $\sim 139^{\circ}$ (Menéndez-Delmestre et al. 2007),

(b) an indication of an oval with a radial extent of $r \sim 50^{\prime \prime}$, basically oriented in north-south direction, and

(c) a $m=2$ spiral pattern that is consistent with a spiral density wave in the inner part and a second spiral pattern that is often referred to as a material wave in the outer part (for details, see Meidt et al. 2013).

At all galactocentric radii, the molecular gas distribution follows the stellar spiral arms very well. Even for the section 



Figure 12. Pixel-by-pixel comparison at 3". 0 resolution of the ${ }^{12} \mathrm{CO}(1-0)$ emission versus tracers of stellar light (from top to bottom): HST H $\alpha$ emission, $3.6 \mathrm{~cm}$ RC, $H S T$ B-band, HST H-band, and the stellar emission at $3.6 \mu \mathrm{m}$. The distribution is shown (from left to right) for the full PAWS FoV and separately for the central $40^{\prime \prime}$, the spiral arms, and the interarm region (as defined in Figure 1). The density is given in a logarithmic color scale.

$25^{\prime \prime} \leqslant r \leqslant 50^{\prime \prime}$, where we see evidence for a stellar oval, the molecular gas roughly resembles the spiral arms. This radial range is also where Henry et al. (2003) and Patrikeev et al. (2006) found deviations from a single pitch angle and we see a lack of recent star formation (see Sections 4.2.3, 4.3.1, and 4.3.3). Further inward, the gas distribution is reminiscent of a starburst ring with two tightly wound spiral arms that start at the tip of the inner nuclear bar. The gas distribution along the inner bar reflects the orientation of the nuclear bar. Taken all together, this strongly suggests that the observed molecular gas distribution is heavily influenced by the galactic potential. In addition, kinetic energy, via non-circular motions or large-scale turbulence, will be injected into the ISM, altering its properties and relative composition. However, a detailed analysis of the dynamical effects is beyond the scope of this paper. Various aspects of how the dynamical environment impacts the properties of the molecular gas are studied in a number of companion papers on the PDFs of the CO emission (Hughes et al. 2013b) and the GMC properties themselves (Colombo et al. 2013a); possible dynamical implications have been derived (Meidt et al. 2013).

It is worth noting that the azimuthal offsets $\Phi$ measured by the polar cc (positive $\Phi$ for star formation tracers and negative $\Phi$ for tracers of the underlying stellar potential) are consistent with a gas flow that is always inside the corotation resonance. This is independent evidence that multiple patterns with different pattern speeds must be present in the PAWS FoV.

\subsection{The Relativistic ISM}

Since molecular gas is thought to be the fuel for star formation, numerous studies have examined the relationship between $\mathrm{CO}$ emission and empirical star formation tracers in 




Figure 13. Azimuthal profiles of the polar cc between ${ }^{12} \mathrm{CO}(1-0)$ intensity and other ISM tracers drawn in the radial range of $30^{\prime \prime}<r<80^{\prime \prime}$ in steps of $4^{\prime \prime}$. 5 : ${ }^{12} \mathrm{CO}(1-0)$ intensity (top left), HST I-H color (top right), MIPS $24 \mu \mathrm{m}$ image (middle left), VLA $20 \mathrm{~cm}$ continuum (middle right), and non-stellar continuum at IRAC $3.6 \mu \mathrm{m}$ and $8 \mu \mathrm{m}$ (bottom). The radial position of each profile is indicated by grayscale and line thickness, from small (black and thin line) to large (light gray and thick line) galactocentric radius. Each profile is normalized to the maximum difference between the measured cc profile and the average $(\langle\mathrm{cc}\rangle)$ signal at that radius.

galaxies. It is well established that far-infrared emission is correlated with the $\mathrm{CO}$ emission on large scales within galaxies (e.g., Devereux \& Young 1990; Young \& Scoville 1991, and references therein), while a correlation between the total $\mathrm{CO}$ and $20 \mathrm{~cm} \mathrm{RC}$ luminosities of galaxies has also been reported (Rickard et al. 1977; Israel \& Rowan-Robinson 1984; Adler et al. 1991; Murgia et al. 2002). The correlation between the $\mathrm{CO}$ and $\mathrm{RC}$ emission has been shown to exist for surface brightness as well as intensity in the Milky Way and for galaxies of diverse Hubble types (e.g., dwarfs and ULIRGS; Adler et al. 1991; Allen 1992; Leroy et al. 2005; Liu \& Gao 2010). Detailed studies with a few 100 pc resolution (Murgia et al. 2005; Paladino et al. 2006) have shown that the relation is linear within galaxies as well as between regions of individual galaxies. The robust correlation down to small spatial scales is intriguing since RC emission is only indirectly linked to star formation. Ionized gas surrounding young high mass stars produces thermal free-free radiation at radio frequencies, but approximately $90 \%$ of the $1.4 \mathrm{GHz}$ emission in a normal starforming galaxy is synchrotron radiation emitted by CR electrons gyrating in magnetic fields. While non-thermal radio emission is also linked to star formation, since supernova remnants (SNRs) from short-lived, high-mass stars are the primary site for CR production in galaxies, less than $10 \%$ of the non-thermal radio emission is due to discrete SNRs; the remaining emission is from electrons that escape their parent SNRs and diffuse into the disk and halo, where they are accelerated by the galactic magnetic field (e.g., Lisenfeld \& Völk 2000).

In Section 4.2.4, we showed that the morphology of the $\mathrm{RC}$ emission closely resembles the $\mathrm{CO}$ distribution within the PAWS FoV, even down to spatial scales of $\sim 100$ to $200 \mathrm{pc}$ (see Figure 2). Moreover, the pixel-by-pixel comparison (see Figure 6, bottom two rows) between the $\mathrm{CO}$ and $\mathrm{RC}$ emission in M51 reveals two relations with slightly different slopes $(\sim 0.7$ and $\sim 0.5$ for the correlations at $20 \mathrm{~cm}$, and $\sim 0.9$ and $\sim 0.7$ for

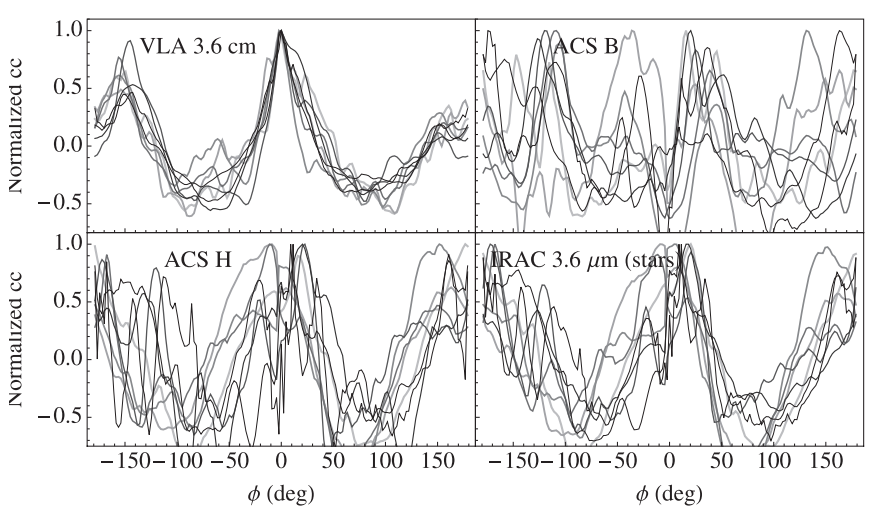

Figure 14. Azimuthal profiles of the polar cc between ${ }^{12} \mathrm{CO}(1-0)$ intensity and SFR and stellar population tracers drawn in the radial range of $30^{\prime \prime}<r<80^{\prime \prime}$ in steps of 4".5: VLA $3.6 \mathrm{~cm}$ continuum (top left), HST B-band (top right), $H S T$ $H$-band (bottom left), and the contaminant-corrected IRAC $3.6 \mu \mathrm{m}$ emission (bottom right). The radial position of each profile is indicated by grayscale and line thickness, from small (black and thin line) to large (light gray and thick line) galactocentric radius. Each profile is normalized to the maximum difference between the measured cc profile and the average $(\langle\mathrm{cc}\rangle)$ signal at that radius.

the correlations at $6 \mathrm{~cm}$ ), which appear to originate from distinct environments within M51's inner disk. Compared with the arm and interarm regions, the RC emission at both 20 and $6 \mathrm{~cm}$ in the central region is brighter for a given $\mathrm{CO}$ flux. This relationship is independent of the emission due to the radio AGN, which is manifested in Figure 6 as a cloud of points that show no relation between $\mathrm{RC}$ and $\mathrm{CO}$ emission.

Potential explanations for the $\mathrm{RC}-\mathrm{CO}$ correlation fall into two broad categories: those in which the correlation is due to a single stellar population that powers both types of emission and those where the correlation arises because the synchrotron emissivity is closely linked to the dense gas distribution. Among the former class of models, Adler et al. (1991) argued for a direct link between $\mathrm{CO}$ emission and non-thermal $\mathrm{RC}$ via $\mathrm{CRs}$ produced by massive star formation. $\mathrm{SNe}$ inject new $\mathrm{CR}$ electrons into the ISM, leading to enhanced synchrotron emission. At the same time, the accompanying CR nuclei (i.e., protons) heat the molecular gas (for a detailed model, see, e.g., Suchkov et al. 1993). Allen (1992) points out that this would imply that the observed $\mathrm{CO}$ brightness is only sensitive to the amount of excitation by CRs and not the intrinsic gas density. As discussed by Murgia et al. (2005), moreover, the observed range of CO intensities would imply gas temperatures above $1000 \mathrm{~K}$ if this scenario is applied. Our finding that the RC in M51 shows no spatial offset from the $\mathrm{CO}$ emission-unlike the hot dust and $\mathrm{H} \alpha$ emission, which presumably trace sites of massive star formation-also argues against this model.

Another possibility is that the good correlation between RC and CO emission in M51's spiral arms is due to enhanced synchrotron emission that arises from the secondary CR electrons produced in the interaction of CRs with the dense molecular gas (as proposed by, e.g., Marscher \& Brown 1978; Murgia et al. 2005; Thompson et al. 2007). In this scenario, interactions between $\mathrm{CR}$ protons and $\mathrm{H}_{2}$ molecules produce (negatively) charged pions whose decay produces secondary (CR) electrons. Thompson et al. (2007) calculate that for gas surface densities of $\Sigma_{\text {gas }} \sim 0.03-0.3 \mathrm{~g} \mathrm{~cm}^{-2}$ (i.e., $\approx 150-1500 M_{\odot} \mathrm{pc}^{-2}$ ), the timescale for CR protons to lose all their energy is significantly shorter than the diffusion timescale to leave the medium. As the observed $\mathrm{H}_{2}$ gas densities in M51 are in this range, this might be a plausible explanation for the very good spatial correlation 
between the $\mathrm{CO}$ emission and $\mathrm{RC}$ in the spiral arms, although the smoother appearance of the RC would point toward some diffusion of the CR electrons from their production sites. While the global spectral index for the entire disk of NGC 5194 is $\alpha_{20-6}=0.9$ (for $S \sim v^{-\alpha}$; Dumas et al. 2011), there are pronounced differences at $15^{\prime \prime}$ resolution with an average $\left\langle\alpha_{20-6}\right\rangle$ of about $0.6,0.8$, and 1.0 for the center, spiral arm, and interarm region, respectively, based on an spectral index map presented by Fletcher et al. (2011, their Figure 7b).

Since the contribution of secondary electrons to the total RC emission (and the ratio between the total $\mathrm{RC}$ and $\mathrm{CO}$ emission) depends sensitively on parameters such as the efficiency of energy transfer from $\mathrm{SNe}$ to $\mathrm{CRs}$, the ratio of primary electrons to nuclei in CRs and the fraction of $\mathrm{CO}$ emission that arises from dense rather than diffuse molecular gas, it is also uncertain whether such a model can explain why such a tight linear $\mathrm{RC}-\mathrm{CO}$ correlation is observed for galaxies with a diverse range of Hubble types. Further analysis, e.g., estimates for the dominant CR cooling processes and timescales for the typical ISM conditions (gas densities, radiation field, magnetic field strength) in different M51 environments and a detailed comparison between the $\mathrm{RC}-\mathrm{CO}$ correlation and the nonthermal radio spectral index, would be required to test whether secondary electron production in molecular clouds helps to explain the close relationship between $\mathrm{CO}$ and $\mathrm{RC}$ emission in some regions of the PAWS field. It is interesting to note that attempts to detect non-thermal RC caused by secondary $\mathrm{CR}$ electrons from Galactic clouds has not been successful so far (e.g., Protheroe et al. 2008, and references therein).

An alternative explanation for the $\mathrm{RC}-\mathrm{CO}$ correlation is that the enhanced $\mathrm{RC}$ emission is due to coupling between the gas density $\rho$ and magnetic field strength $B$, via $B \propto \rho^{\beta}$. That is, since the flux density of the non-thermal radio emission $S_{1.4, \text { nth }}$ at $1.4 \mathrm{GHz}$ may be written (e.g., Hoernes et al 1998) as

$$
S_{1.4, \text { nth }} \propto n_{\mathrm{CR}} l_{\mathrm{CR}} B^{1-\alpha_{\mathrm{nth}}},
$$

an increase in the RC emission may be linked to an increased magnetic field strength $(B)$, rather than an increase in the number of $\mathrm{CR}$ electrons. In this equation, $l_{\mathrm{CR}}$ is the path length through the synchrotron-emitting region along the line-of-sight and $n_{\mathrm{CR}}$ is the volume density of CR electrons with energy between $E$ and $E+\delta E$, for an injection CR energy spectrum $N(E)=N_{0} E^{-p}$, where $\alpha_{\text {nth }}=-p / 2$. The expression in Equation (1) is fairly general, as it allows for variations in the scale height of the synchrotron disk and does not assume a relationship between $B$ and $n_{\mathrm{CR}}$.

As an explanation for the local RC-CO correlation, the advantage of $\rho-\beta$ coupling models is that the physical mechanism responsible for the coupling between the magnetic field and gas volume density is truly local in nature, involving a relationship between the magnetic field and charge density within (nearly) neutral gas. Observations indicate $\beta \sim 0.5 \pm 0.1$ globally and within galactic disks, even on the scale of individual clouds ( 100 pc, e.g., Fiebig \& Guesten 1989; Berkhuijsen et al. 1993; Niklas \& Beck 1997). This range of $\beta$ values is in good agreement with results from turbulent magnetohydrodynamic simulations $(\beta=0.4-0.6$, e.g., Ostriker et al. 2001; Cho \& Vishniac 2000; Groves et al. 2003) and with equipartition between magnetic and turbulent energy densities in the ISM $(\beta=0.5$, e.g., Ko \& Parker 1989). Regardless of the exact value of $\beta, B-\rho$ coupling yields

$$
S_{1.4, \text { nth }} \propto n_{\mathrm{CR}} l_{\mathrm{CR}} \rho^{\beta\left(1-\alpha_{\mathrm{nth}}\right)}
$$

for the correlation between the non-thermal $1.4 \mathrm{GHz}$ emission and the gas volume density. The gas surface density $\Sigma_{\text {gas }}$ is simply the projection of the volume density through the disk, so for a gas disk with constant scale height $l_{\text {gas }}$ we would expect to observe a power-law scaling with a similar exponent, i.e.,

$$
S_{1.4, \text { nth }} \propto n_{\mathrm{CR}} l_{\mathrm{CR}} \Sigma_{\mathrm{gas}}^{\beta\left(1-\alpha_{\mathrm{nth}}\right)} \text {. }
$$

Since the interstellar gas in M51's inner disk is almost entirely molecular, we can replace $\Sigma_{\text {gas }}$ in Equation (3) with $I_{\mathrm{CO}}$. The relationship between the surface density of CRs, $N_{\mathrm{CR}}=n_{\mathrm{CR}} l_{\mathrm{CR}}$, and $\Sigma_{\text {gas }}$ is somewhat uncertain (see, e.g., the discussion in Murgia et al. 2005). In general terms, regions with high gas surface density are linked to regions with high levels of star formation activity. On one hand, high star formation rates (SFRs) would tend to increase the supply of CR electrons, but on the other hand may lead to higher rates of convective escape. Assuming that the surface density of CRs $N_{\mathrm{CR}}$ is independent of $\Sigma_{\text {gas }}$, then for $\beta=0.5$ and $\alpha_{\text {nth }}=-0.7$, we obtain a slightly sub-linear RC-CO correlation, i.e., $S_{1.4, \text { nth }} \propto I_{\mathrm{CO}}^{0.85}$, in general agreement with our pixel-by-pixel correlations in Figure 6. The different slope and vertical offset between the $\mathrm{RC}-\mathrm{CO}$ correlations in the center and disk of M51 could be due to the higher level of ongoing massive star formation in the central region. That is, fresh $\mathrm{CR}$ electrons from recent $\mathrm{SNe}$ increase $N_{\text {CR }}$ (e.g., Tabatabaei et al. 2007), while the shallower slope is consistent with the shallower radio spectral index in the central region (which itself may be due to a higher rate of convective escape via a galactic wind or the fact that the CR electrons are still relatively "warm"). The presence of a few points scattered along the shallower relation in the spiral arm plot is consistent with this interpretation, since the brightest RC peaks coincide with the brightest $24 \mu \mathrm{m}$ emission (see Section 4.2.4).

The detailed analysis of the magnetic fields and RC emission in M51 by Fletcher et al. (2011) found that compression of the magnetic field by a spiral arm shock would significantly overpredict the observed moderate contrast between the arm and interarm regions. Therefore, they concluded that no strong spiral arm shock is compressing the magnetic field, consistent with the analysis of Meidt et al. (2013) who, based on a kinematic analysis of the $\mathrm{CO}$ emission, find no evidence for a strong dynamical shock. However, higher angular resolution and more sensitive RC observations as now possible with the upgraded Karl G. Jansky VLA would be required to further test if compression of the magnetic field is indeed not important even on the small scales probed by our CO data.

\subsection{Radiation Field}

The radiation field within the PAWS FoV of M51 contains-broadly speaking-three main sources: the ISRF dominated by the old stellar population, young active star forming regions with a significant energy increase at short wavelengths (i.e., UV continuum), and the AGN itself that displays a jet feature (e.g., Maddox et al. 2007). The spatial distribution of these three components varies not only radially, but also with respect to the geometry of the non-axisymmetric components (e.g., bar, spiral) of the gravitational potential.

The impact of radiation on molecular gas is typically described in the form of a photodominated (or photodissociation) region (PDR) that is nowadays defined as a region where FUV photons dominate the energy balance or chemistry of the gas (e.g., Tielens 2008). Thus, PDRs are not only associated with $\mathrm{H}$ II regions, but also the diffuse ISM, i.e., anywhere that FUV 
photons penetrate. PDRs in a broad sense are the transition region from the ionized to the molecular phase of the gaseous ISM. Energetic UV photons from nearby hot stars (e.g., within a $\mathrm{H}$ II region) cause photoionization and photodissociation in the outer layer of a molecular cloud. Due to attenuation of these photons by atoms, molecules, and dust grains, the following (stratified) structure exists from outside to inside, i.e., lower to higher gas densities: (1) a $\mathrm{H}^{+} / \mathrm{H}$ transition zone followed by an atomic $\mathrm{HI}_{\mathrm{I}}$ layer and (2) the $\mathrm{HI} / \mathrm{H}_{2}$ transition zone after which hydrogen is molecular; within the molecular $\mathrm{H}_{2}$ gas, the transition from ionized carbon to $\mathrm{CO}(\mathrm{C} \mathrm{II} / \mathrm{C} \mathrm{I} / \mathrm{CO})$ occurs at slightly higher gas densities. However, the structure of GMCs is far from being simple spheres; recent Galactic observations show that GMCs actually contain filaments of varying density structures (e.g., André et al. 2010; Pety \& Falgarone 2000), consistent with expectation based on the ISM's turbulent nature (e.g., Hennebelle \& Audit 2007, 2008). Therefore, it is observationally very challenging on the size scales considered here to discriminate between GMCs that are full PDRs (as in the center of Maffei 2; Meier \& Turner 2012) or only have PDR surface layers (as in the center of IC 342; Meier \& Turner 2005). A more detailed overview on the heating and cooling processes occurring in the molecular gas is provided in Appendix A.

\subsubsection{The Old Stellar Population (ISRF)}

The ISRF is mainly caused by the old underlying stellar population(s). As the stellar density of the old population is changing from the bulge to the disk, as evident from, e.g., the optical-to-near-infrared radial profiles (Muñoz-Mateos et al. 2011), so must the incident radiation field. In the following, we discuss the role of the ISRF component that is dominated by the old stellar population in the relationship between the molecular gas and dust emission (i.e., $24 \mu \mathrm{m}, \mathrm{PAH}$ ).

Hot dust (very small grains). As the radiation of the old stellar population is significantly less energetic than that emitted by hot OB stars, it is not clear how much it can affect the properties of the $\mathrm{CO}$-identified molecular clouds that typically have extinction values of $A_{V}>1$ (see Section 4.2.2 for details). The radial variation in the old stellar population can explain the different slopes seen between $24 \mu \mathrm{m}$ emission and $\mathrm{CO}$ flux. The shallow slope seen in the spiral arms (Figure 6, middle-right panel in the second row) can be attributed to diffuse $24 \mu \mathrm{m}$ "cirrus" emission (for a detailed discussion, see Section 5 of Leroy et al. 2012) that is caused by heating from the ISRF. That is, we see the short-wavelength tail of the blackbody emission of the cold dust component that is well mixed with the molecular gas and not hot dust heated by young massive stars. This immediately implies that in the center and in certain areas of the spiral arms the $24 \mu \mathrm{m}$ emission is "over"-luminous compared with the CO emission. Muñoz-Mateos et al. (2009) studied the radial profile of the dust emission in the SINGS sample, including M51. They find that M51's dust luminosity surface density and derived dust mass surface density vary independently with radius, with the dust in the central $2 \mathrm{kpc}$ being significantly more luminous for a given mass surface density (their Figure 7.44). Young star forming regions are very good candidates for causing this "over" luminosity.

However, the situation in the central disk is more complex. While it is very likely that within the molecular ring, the ongoing massive star formation (providing significant UV photons) is the main heating source for the dust, and thus the enhanced $24 \mu \mathrm{m}$ emission, it is not obvious inside the molecular ring what is causing the excess $24 \mu \mathrm{m}$ emission (best seen at $70 \mu \mathrm{m}$ in
Figure 2, middle-right panel). This enhanced 24(70) $\mu \mathrm{m}$ emission is indicative of dust hotter than that seen in the spiral arms, and indeed Mentuch Cooper et al. (2012) find that the dust temperature in the center is about $25 \mathrm{~K}$, only slightly lower in the (dust) spiral arms and about 3-4 K colder in the disk unaffected by recent star formation. The increase in dust temperature inside the molecular ring could either come from an increase in stellar UV emission or the AGN. Unfortunately, the (far-)infrared imaging from Herschel used by Mentuch Cooper et al. (2012) does not have sufficient spatial resolution to spatially resolve the central region. An obvious candidate for the stellar radiation field would be the older stars that make up the bulge of M51 that dominates over the central disk for radii $r \leqslant 15^{\prime \prime}$ (Beckman et al. 1996). Lamers et al. (2002) derive an age of more than 5 Gyr for the prominent "smooth, yellowish" component that they attribute to be the bulge. Recently, Groves et al. (2012) showed that the old stellar population in the bulge of M31 alone can explain the observed increase in dust temperature without the need for any young ( $\leqslant 100 \mathrm{Myr}$ ) stars. Similarly, Bernard et al. (2008) observe an increase in dust temperature at the center of the LMC where the density of old stellar populations is enhanced due to the stellar bar. The relation of $\mathrm{CO}$ emission and emission from the old stellar population (as traced by its $H$-band and contaminantfree $3.6 \mu \mathrm{m}$ emission; see Section 4.3.4) shows a remarkable difference for the center and the disk (arm and interarm region): the center exhibits a clear linear relation, while the latter are basically consistent with no correlation. This could potentially imply that the molecular gas might experience additional heating by the enhanced ISRF caused by the higher density of old stars.

PAHs. Given the excellent correlation between $\mathrm{CO}$ and $\mathrm{PAH}$ emission, we suggest in the following that the ISRF that is dominated by the old stellar population is the underlying cause for this correlation. Modeling of the infrared spectral energy distribution with the Draine \& Li (2007) model using the PACS 70 and $160 \mu \mathrm{m}$ data in conjunction with the SPIRE $250 \mu \mathrm{m}$ imaging following the methodology of Aniano et al. (2012) shows that the ISRF in M51 is (within a factor of two) about 11 times, 7 times, and 6.5 times the Galactic value in the central, spiral arm, and interarm region of the PAWS area (B. Groves, 2013, private communication), respectively, so likely sufficient to excite PAH emission.

Although the exact excitation channels and conditions for PAH emission observed in the mid-infrared spectra of nearby galaxies are not well understood (e.g., Smith et al. 2007), it is well-established that significant PAH emission (similar to the mid-infrared continuum arising from heated small grains) is related to star forming regions ranging from PDRs to H II regions (see the review by Tielens 2008). Photoelectric heating by UV photons from these star forming regions or UV photons present in the ISRF are very likely the main excitation mechanism (Tielens 2008). Heitsch et al. (2007) investigated the predictive power of PAH emission for cloud structure and its underlying density distribution using a single cloud illuminated by a single $\mathrm{O}$ star. They find that for diffuse gas where UV photons can penetrate the entire cloud, a good correlation between PAH emission and gas density distribution is expected. However, at higher gas densities, UV photons no longer illuminate the full cloud but only its "rims" and thus a misinterpretation of the underlying gas distribution will become possible and most likely. Based on these theoretical findings, the correlation between $\mathrm{PAH}$ and $\mathrm{CO}$ emission will depend on both the exact density distribution of the (GMC) structure and 
the distribution of the incident radiation field. This implies that a spatial correlation between $\mathrm{CO}$ emission and $\mathrm{PAH}$ emission should exist, however, the observed intensities might not agree, particularly in regions of intense star formation.

Our observational results showing a very good spatial relation between $\mathrm{CO}$ and $\mathrm{PAH}$ emission corroborate this interpretation. Two of our findings are interesting in this context. PAH emission is strong even in regions where no excess UV flux from nearby massive star formation is present, like the "gaps" in the spiral arms seen in, e.g., UV emission (see Section 4.3.3). In addition, there is tentative evidence that the geometry of the $\mathrm{CO}$ distribution, especially in the interarm region, is very similar to the geometry of the PAH emission (se Section 4.2.5). However, the pixel-by-pixel comparison of both PAH emission features shows no predictive power in the disk, i.e., the PAH strength varies a lot for a given $\mathrm{CO}$ flux. Only in the center is a tighter correlation present. This points toward a strong link between PAH emission and molecular clouds. Although excess UV radiation from slightly older stars (up to $\sim 100 \mathrm{Myr}$ ) could increase the PAH emission by providing a stronger (local) radiation field, we find no difference between the arm and interarm regions when considering the range of $\mathrm{PAH}$ emission probed. Thus, the absence of a correlation in the disk (arm, interarm) suggests that the PAH emission is not tracing the true gas density, but rather arises from cloud surfaces in the disk of M51 while the incident radiation field is not very important or PAHs are readily destroyed. The apparent correlation in the center between PAH and $\mathrm{CO}$ emission is at least partially due to the contribution of hot dust emission to the non-stellar emission at $3.6 \mu \mathrm{m}$ and $8 \mu \mathrm{m}$ as the ratio of $24 \mu \mathrm{m}$ and non-stellar $8 \mu \mathrm{m}$ versus $\mathrm{CO}$ flux exhibits a shallower slope.

Sandstrom et al. (2010) investigated the spatial relation between PAH emission and other dust and gas tracers in the Small Magellanic Cloud and noted that PAH emission shows the best correspondence with $\mathrm{CO}$ emission tracing molecular gas. They offered two explanations for this correlation: either PAHs are formed in molecular clouds or the shielding offered by the dense gas environments prevents the destruction of the PAH features. Our good spatial correlation between PAH and $\mathrm{CO}$ emission in all environments underlines the close relation between PAHs and molecular gas. A similar conclusion has been reached for three clouds in IC 10 by Wiebe et al. (2011). Taking all this evidence together, this strongly suggests that PAHs might indeed form in molecular gas or at least the dense phase of the neutral ISM and that the ISRF is illuminating the PAHs in the outer shell of GMCs. Reversely, it directly implies that maps of, e.g., the $8 \mu \mathrm{m}$ PAH emission have a very good predictive power of the expected spatial distribution of the molecular line emission traced by $\mathrm{CO}(1-0)$, however, not the expected line strength. As a corollary, this implies that $8 \mu \mathrm{m}$ PAH emission should have a poor predictive power of the recent SFR (at high spatial resolution). It should work fine at $\sim \mathrm{kpc}$ resolution where $\mathrm{CO}$ versus star formation is robust.

\subsubsection{The OB Stars (Massive Star Formation Regions)}

The distributions of the molecular gas and the sites of ongoing and/or recent star formation, as traced by $\mathrm{H}$ II regions, young stars, and stellar clusters, show a correlation on the scale of galactic structures, e.g., the spiral arms and the central gas ring. However, they do on average not spatially coincide when compared at $\sim 1^{\prime \prime}$, i.e., $37 \mathrm{pc}$, resolution (see Sections 4.3.1 and 4.3.3). This is expected as the strong UV radiation from the young stars and their stellar winds are believed to quickly disrupt the parent cloud (e.g., Hopkins et al. 2010 and references therein). In this context, it is interesting to distinguish between the star forming sites offset from the dense molecular gas along the gas spiral arms and the star forming ring where regions of young stars and dense molecular gas are well mixed on $100 \mathrm{pc}$ scales. In the former case, the expectation is that one observes the properties of a PDR not diluted much by contributions from the cold ISM component whereas the ring provides an ideal situation to obtain global properties of PDRs mixed together with cold molecular gas.

Spiral arms. The azimuthal offset of all star formation tracers from the CO spiral arms is on average at least $\Phi \sim 10 \mathrm{deg}$ for radii $r>50$ " (i.e., past the "gap"; see Figure 5). This translates into a spatial separation of over $300 \mathrm{pc}$ for radii beyond $1.84 \mathrm{kpc}$ between the sites of massive star formation, and thus OB stars, and the reservoir of molecular gas. Although the impact radius of giant $\mathrm{H}$ II regions onto surrounding GMCs is not very well known, e.g., Wilson et al. (1997) quote an upper limit of $<120 \mathrm{pc}$ based on a detailed study of GMCs with associated $\mathrm{H}$ II regions in M33, immediately implying that the radiation of young stars is not affecting the bulk of the molecular gas in the spiral arms. It is interesting that emission from the expected dissociation products such as $[\mathrm{C} \mathrm{II}]$ and $\mathrm{H}_{\mathrm{I}}$ is found off the dense gas arms, especially the northern arm (see Section 4.2.1). Similar to the [C II] (and $\mathrm{HI}$ ) distribution, the hot dust emission is present in distinct locations, also providing further evidence that the UV radiation of the young stars is impacting the ISM, as evidenced by the higher dust temperature, offset from the $\mathrm{CO}$ arms (see Figure 10 of Mentuch Cooper et al. 2012). The spatial resolution of the infrared data is, unfortunately, not high enough to derive exact source sizes. However, comparison with the hot dust distribution and the location of the H II regions suggests that the impact is fairly localized-consistent with previous findings by Wilson et al. (1997). If we take the $\mathrm{H} \alpha$ emission as our best predictor of the location where radiative and mechanical energy released by young OB stars is impacting the molecular gas, stellar feedback should not strongly alter the properties of the bulk of the molecular gas located in the spiral arms. However, this does not rule out the existence of individual regions in the disk where stellar feedback has likely significantly impacted the molecular gas, e.g., northeast of the molecular ring inside the northern spiral arm.

Star forming ring. In the molecular ring, hot young stars and cold molecular gas are very close to each other, if not spatially coincident. Therefore, it is interesting to test if this massive ongoing star formation leaves a significant imprint on the molecular gas. The northwestern ring segment is brightest in $\mathrm{CO},[\mathrm{C} \mathrm{II}]$, and $24 \mu \mathrm{m}$ emission, suggesting that star formation might have the strongest impact onto the properties of the molecular gas. The spatial coincidence of these three tracers means that photodissociation and dust heating by young stars is occurring. How much this excess UV radiation is affecting the cold molecular gas is not straightforward to judge. Therefore, it is illustrative to compare the surface density of the cold molecular gas with that of the warm and hot $\mathrm{H}_{2}$, as derived from Spitzer/InfraRed Spectrograph (IRS) imaging by Brunner et al. (2008). They find that the $\mathrm{H}_{2}$ surface density of both the warm $(100-300 \mathrm{~K})$ and hot $(400-1000 \mathrm{~K})$ phase is highest at the northwestern part of the molecular ring. A comparison with the cold $\mathrm{H}_{2}$ surface density (probed by our $\mathrm{CO}$ data and derived assuming a Galactic conversion factor) of $\sim 1000 \mathrm{M}_{\odot} \mathrm{pc}^{-2}$ shows that the warm phase makes up about $1 \%$ while the hot phase is less than $0.02 \%$ of the cold molecular gas surface density. We find for the outer northern spiral arm in the PAWS field 
that is covered by the IRS $\mathrm{H}_{2}$ footprint (see Figure 1 of Brunner et al. 2008) similar values within a factor of two. This implies that the OB stars are not sufficient to heat large fractions of the molecular gas above $100 \mathrm{~K}$. For instance, using observations from multiple CO transitions, Weiss et al. (2001) derive an average kinetic temperature of $T_{\text {kin }} \geqslant 100 \mathrm{~K}$ for the molecular gas associated with the central starburst in the nearby galaxy M82. Thus, we speculate that most of the molecular gas in the star forming ring of M51 is still in the cold $(<100 \mathrm{~K})$ phase despite the presence of strong UV heating by young stars. This would imply that the massive star formation is not significantly altering the properties of the cold molecular gas. However, a more detailed and proper analysis using multiple $\mathrm{CO}$ transitions is required to firmly test this.

In summary, we find no strong evidence that recently formed massive stars are significantly impacting the bulk of the molecular gas reservoir in the PAWS FoV.

\subsection{3. $A G N$}

There is no strong evidence that the AGN is altering the properties of the cold molecular gas on large scales. Scoville et al. (1998) already noted that the nuclear molecular gas reservoir as seen in its $\mathrm{CO}(1-0)$ emission line is concentrated in a ring-like distribution with a size of $\sim 100 \mathrm{pc}$. This nuclear gas concentration brightens in higher $\mathrm{J}$ transitions of the $\mathrm{CO}$ molecule, indicating higher excitation temperatures (Matsushita et al. 2004). Strong HCN(1-0) line emission is detected from this reservoir (Kohno et al. 1996; Helfer \& Blitz 1997), further indicating that the AGN is altering the molecular gas properties here. Based on a detailed kinematic analysis of high angular resolution $\mathrm{CO}(2-1)$ data, Matsushita et al. (2007) suggested that the molecular gas is entrained by the jet.

A jet-like structure has been observed emanating from the nucleus at X-ray and radio wavelengths with an extent of about $6^{\prime \prime}$ to the south and $13^{\prime \prime}$ to the north (e.g., Crane \& van der Hulst 1992; Terashima \& Wilson 2001; Maddox et al. 2007). Ford et al. (1985) suggested, based on optical line ratios and their unusual width, that the line emission is caused by shocks where the bidirectional jet interacts with disk material. This interpretation is consistent with the properties of the X-ray gas and the RC that are both suggesting a non-stellar source for their excitation (Maddox et al. 2007). Careful comparison of the distribution of the CO line emission, the optical extinction, and the $20 \mathrm{~cm} \mathrm{RC}$ (Figure 2) shows that the CO emission is closely following the optical dust lanes that are reminiscent of dust lanes regularly found along the leading side of large-scale bars. However, no obvious spatial correlation to the jet geometry (as seen in its $20 \mathrm{~cm}$ continuum) is found. We see a lack of CO emission at the location of the southern bubble, which could be interpreted as the jet dissociating molecular disk material. Targeted sensitive molecular line observations would be necessary to judge if the jet has indeed had an impact on the molecular gas in the disk. No evidence for effects of the jet is found outside a radius of $\sim 6^{\prime \prime}$ and off the jet features. Thus, we conclude that the jet is basically not affecting the molecular gas in the central region despite its extra X-ray and UV emission contribution to the radiation field.

It is worth noting that Brunner et al. (2008) find that the hot (400-1000 K) $\mathrm{H}_{2}$ gas is peaking at the nucleus. Interestingly, the nuclear distribution of the hot gas phase is elongated along the radio jet axis in the northern direction. The authors speculate that heating by the $\mathrm{AGN}$ is causing this excess emission.

\subsection{The Relation between Molecular Gas and (Cold) Dust}

The excellent correspondence between the distribution of the $\mathrm{CO}$ emission and the optical extinction as traced by the $I-H$ color implies that the molecular gas and cold dust are at least cospatial and very likely well mixed. This is in agreement with the large body of work on Galactic molecular clouds. Previous work (Bohlin et al. 1978; Lombardi et al. 2006) found that for extinction values below (1-2) mag no $\mathrm{CO}$ emission is detectable (on GMC scales), in agreement with the expectations from models for photodissociation of the $\mathrm{CO}$ molecule (e.g., van Dishoeck \& Black 1988) where the shielding of CO requires certain column densities. Studies of resolved Galactic clouds using extinction measures of $A_{V}$ from individual stars with nearinfrared colors typically find that the ${ }^{12} \mathrm{CO}(1-0)$ emission has an almost linear relation to $A_{V}$ for $2 \mathrm{mag} \leqslant A_{V} \leqslant(4-6) \mathrm{mag}$, while for large extinction values the ${ }^{12} \mathrm{CO}$ emission appears to saturate (Lombardi et al. 2006; Pineda et al. 2008; Heiderman et al. 2010).

Although within the PAWS FoV the morphology is very similar, the correlation between the $\mathrm{CO}$ intensity and $I-H$ color is not constant (see Figure 6, top row). The slope is changing between the environments, most notably from the center to the disk. A change in the color of the underlying stellar population can be excluded to explain the different slopes as no radial change in color has been observed (e.g., $I-H$ color by Rix $\&$ Rieke 1993) nor a trend in age for the (old) stellar population (Mentuch Cooper et al. 2012). Thus the observed change in slope can be either caused by a change in the properties of the obscuring material, i.e., the dust, or a change in the excitation conditions of the $\mathrm{CO}$ emission line. Indeed, the dust temperature as well as the ISRF are higher in the center than in the disk (with the exception of the spiral arms; Mentuch Cooper et al. 2012). Pineda et al. (2008) also observed different slopes as a function of environment within one single GMC (Perseus cloud), while Heiderman et al. (2010) find slightly different dependencies between $A_{V}$ and $\mathrm{CO}$ intensity for the two GMCs they analyzed. This might indicate that the relation between $A_{V}$ and $\mathrm{CO}$ intensity depends on several parameters of the local environment.

In order to better understand and quantify these differences, detailed modeling of the stellar spectral energy distribution is important. In any case, the observed linearity between $I-H$ color and CO intensity shows that the gas and dust are well mixed and that there is a preferred gas-to-dust ratio within M51, as found by Mentuch Cooper et al. (2012).

Finally, we note that the correlation between the tracers of hot dust and the $\mathrm{CO}$ emission is not very good in every environment. Since the dust-to-gas ratio is basically constant around a value of 105 over the PAWS FoV (Mentuch Cooper et al. 2012), the simplest explanation is that the heating source of the dust as observed at $24 \mu \mathrm{m}$ (and $70 \mu \mathrm{m}$ ) is varying and therefore so is the luminosity of the dust. Obvious examples are the sites of young star formation that can explain the azimuthal offset between the molecular gas arms and the $24 \mu \mathrm{m}(70 \mu \mathrm{m})$ emission along these arms.

\section{THE IMPORTANCE OF GALACTIC ENVIRONMENT FOR MOLECULAR GAS PROPERTIES}

The detailed comparison of $\mathrm{CO}$ emission with other tracers of the ISM and stellar population(s) at cloud-scale resolution reveals that the relations are changing as a function of galactic 
environment, i.e., between the disk and the bulge, but also the arm and interarm regimes. It is interesting that we find no close or simple correlation between the presence of star formation and the distribution of the cold molecular gas. Two interesting aspects are the gaps observed between the central disk and the spiral arms, especially in the UV emission, and the "clumping" of stellar clusters (clearly) seen along the northern spiral arm with roughly equidistant separations. Close inspection of the locations of the clusters of stellar clusters show that they are associated with spurs in the molecular gas distribution. This will be discussed in detail in a forthcoming paper.

We find that massive star formation is not always found at or even close to the brightest $\mathrm{CO}$ flux peaks (presumably also peaks in gas density), i.e., the gaps in star formation tracers between the center and the spiral arms. As the gaps are also apparent in tracers of younger stellar populations (H II regions, embedded star formation), this suggests that no massive star formation has been occurring at this particular radial range for several tens of Myr. This situation is reminiscent of the prominent dust and gas lanes found along bars that also show no strong star formation (Sheth et al. 2002). However, in the case of M51, strong shocks cannot be invoked to explain this difference. Meidt et al. (2013) explain this behavior by pressure support of GMCs that prevents the collapse and thus subsequent star formation.

Clearly, the underlying gravitational potential is significantly affecting the molecular gas distribution. The spatial offset from the convex side of the spiral arms can be explained by the presence of a spiral density wave (e.g., Vogel et al. 1988). To some level, an offset between H II regions and their birthplace, molecular gas clouds, is expected. Both Schruba et al. (2010) and Onodera et al. (2010) report that the relation between (extinction-corrected) $\mathrm{H} \alpha$ emission and $\mathrm{CO}$ emission in M33 breaks on scales below $\approx 300 \mathrm{pc}$-similar to our observed angular separation between molecular gas and $\mathrm{H}$ II regions. They argue that the time evolution of the GMCs themselves is adding significant scatter, as spatial offsets caused by a spiral density wave can be ignored in the case of M33. Another point raised by Onodera et al. (2010) is that the $\mathrm{H} \alpha$ emission does not necessarily trace the ionizing stars, but rather the shell of the $\mathrm{H}$ II regions, and therefore offsets are to be expected. As Galactic GMCs also show a wide spread of more than two orders of magnitude in their star formation activity (Mooney \& Solomon 1988; Mead et al. 1990), the lack of a relation between $\mathrm{H} \alpha$ and $\mathrm{CO}$ emission at $110 \mathrm{pc}$ resolution is not that surprising. However, the current data do not allow us to discriminate between an evolutionary delay or the fact that only a fraction of the molecular gas will form stars.

Despite the massive star formation present in the PAWS FoV, we find no strong evidence for a general impact of this star formation on the cold molecular gas, as probed by the $\mathrm{CO}$ emission. In particular, the relations between $\mathrm{CO}$ flux and ISM/stellar tracers are always very similar for the arm and interarm region, arguing against a strong effect from stellar feedback, both radiative as well as mechanical, onto the molecular gas over the scales probed here (about 40-100 pc). Alternatively, the high molecular gas surface density in M51 could prevent a large propagation/penetration into the molecular gas phase. In order to thoroughly test such feedback scenarios, more detailed studies of other nearby galaxies with high quality, high angular resolution data are required.

\section{SUMMARY AND CONCLUSIONS}

We present a multi-wavelength comparison between the molecular gas distribution and other tracers of the ISM and stellar components at cloud-scale resolution in the central $9 \mathrm{kpc}$ of the disk of the grand-design spiral galaxy M51. For our study, we utilize new $\sim 1^{\prime \prime}$ resolution $\mathrm{CO}(1-0)$ imaging from the PAWS project in conjunction with existing archival data from the UV to radio regimes. In particular, we find the following.

1. Based on the good spatial correlation of low-frequency RC and $\mathrm{CO}$ line emission, we argue for a physical relation between $\mathrm{RC}$ and $\mathrm{CO}$ emission that does not directly depend on the formation/presence of massive stars. This immediately implies that (at least in certain galactic environments) the relation between $\mathrm{CO}$ line emission and $\mathrm{RC}$ is more fundamental than between $\mathrm{CO}$ emission and IR emission. We show that the relation between magnetic field strength and gas density expected from equipartition can explain our observations.

2. The good spatial correlation between $\mathrm{CO}$ and PAH emission seen in the entire PAWS FoV suggests that PAH molecules are closely associated with molecular gas and that even the old stellar population is providing sufficient excitation in the interarm region. However, the poor correlation of flux between $\mathrm{CO}$ and $\mathrm{PAH}$ emission implies that PAH emission is a poor predictor of the gas mass and most of the PAH emission is only arising from the surfaces of GMCs.

3. The spatial correlation between molecular gas and ongoing massive star formation is complex and strongly depends on galactic environment (i.e., the underlying gravitational potential), showing coincidence, offsets, and even absence. For the spiral arm, we conclude that due to the large average minimum spatial separation of $\sim 300$ pc between the molecular gas arms and the sites of massive star formation, no profound impact onto the properties of the molecular gas is expected, consistent with other observations presented here. The situation in the star forming ring is less clear. A comparison with Spitzer mid-IR $\mathrm{H}_{2}$ observations hints that the conditions are not as extreme as in the centers of nearby starburst galaxies.

4. The impact of the AGN on the molecular gas in the disk of M51 is not strong and appears to be restricted to a small region around the nucleus.

5. The optical-near-IR color $I-K$ provides a very good mapping of the distribution of the molecular gas and shows a good correlation with the $\mathrm{CO}$ emission, but with a changing slope as a function of galactic environment, however.

This study is the first in a series of papers investigating the molecular gas at GMC resolution in the disk of M51 from PAWS. It clearly demonstrates that future studies of galactic disks with ALMA will provide new insights into the physics of the ISM, as well as the star formation process.

We thank the IRAM staff for their support during the observations with the Plateau de Bure Interferometer and the $30 \mathrm{~m}$ telescope. E.S. thanks Kevin Croxall for reducing and providing the Herschel PACS [C II] map, Hendrik Linz for the reduction of the Herschel PACS imaging at 70 and $160 \mu \mathrm{m}$, and Frank Bigiel for providing the GALEX images used. E.S. is grateful for many useful discussions with Brent Groves. D.C. and A.H. acknowledge funding from the Deutsche Forschungsgemeinschaft (DFG) via grant SCHI 536/5-1 and 
SCHI 536/7-1 as part of the priority program SPP 1573 "ISMSPP: Physics of the Interstellar Medium.” C.L.D. acknowledges funding from the European Research Council for the FP7 ERC starting grant project LOCALSTAR. T.A.T. acknowledges support from NASA grant \#NNX10AD01G. During this work, J.P. was partially funded by the grant ANR-09-BLAN-0231-01 from the French Agence Nationale de la Recherche as part of the SCHISM project (http://schism.ens.fr/). E.S., A.H., and D.C. thank the NRAO for support and hospitality during their visits in Charlottesville and Socorro. E.S. thanks the Aspen Center for Physics and the NSF grant \#1066293 for hospitality during the development and writing of this paper. This research has made use of the NASA/ IPAC Infrared Science Archive, which is operated by the Jet Propulsion Laboratory, California Institute of Technology, under contract with the National Aeronautics and Space Administration. S.G.B. acknowledges economic support from Junta de Andalucia grant P08 TIC 03531. The National Radio Astronomy Observatory is a facility of the National Science Foundation operated under cooperative agreement by Associated Universities, Inc.

Facilities: IRAM (PdBI), IRAM (30 m), HST (ACS), HST (NICMOS), GALEX, NRAO (VLA), Herschel (PACS), Spitzer (IRAC), Spitzer (MIPS)

\section{APPENDIX A}

\section{HEATING AND COOLING OF MOLECULAR GAS}

Here, we provide a brief summary of the heating and cooling mechanisms of molecular gas in (star forming) galaxies and discuss implications for the observed CO line emission and the relation between $\mathrm{CO}$ intensity and $\mathrm{H}_{2}$ mass. For the purpose of this paper, we focus on the cold dense molecular gas (with $\left.n\left(\mathrm{H}_{2}\right)>10^{2} \mathrm{~cm}^{-3}, T<100 \mathrm{~K}\right)$ that is normally assumed to be present in GMCs. We make use of these findings in the discussion of our cloud-scale relations (see Section 5) and environmental effects (see Section 6).

\section{A.1. Molecular Hydrogen}

There are several excitation avenues for $\mathrm{H}_{2}$ that contribute differently depending on the exact physical properties of the gas (e.g., density, temperature, metallicity, dust-to-gas ratio) and its surroundings (radiation field, CR density, shocks), which are both expected to vary across a galactic disk.

\section{A.1.1. Cosmic Ray Heating}

The interaction between CRs - mainly protons and electrons accelerated to $\mathrm{GeV}$ energies and beyond-and $\mathrm{H}_{2}$ molecules is the prime heating source for the molecular ISM (in the absence of nearby ongoing star formation) and is proportional to the $\mathrm{H}_{2}$ density $n\left(\mathrm{H}_{2}\right) \times$ the $\mathrm{CR}$ ionization rate $\zeta_{\mathrm{CR}}$ (Goldsmith \& Langer 1978). CR electrons and protons (above $10 \mathrm{MeV}$ ) photoionize $\mathrm{H}_{2}$ molecules (as these are the most abundant particles). The free electrons collide with other $\mathrm{H}_{2}$ molecules and transfer some of their kinetic energy. In addition, $\mathrm{H}_{3}^{+}$is formed from the reaction of $\mathrm{H}_{2}^{+}+\mathrm{H}_{2} \rightarrow \mathrm{H}_{3}^{+}+\mathrm{H}$. Dissociative recombination into $\mathrm{H}_{2}$ is a major source of heating for the case of dense molecular gas (see, e.g., Figure 2 of Maloney et al. 1996). As most $\mathrm{CR}$ protons and electrons can completely penetrate molecular clouds, as evidenced by $\gamma$-ray emission from molecular clouds (e.g., Bloemen et al. 1986), ${ }^{19}$ this heating mechanism is expected

\footnotetext{
19 The interaction between CRs and (atomic and) molecular gas can be observed via the $\gamma$-ray emission that results from $\pi^{\circ}$ decay and bremsstrahlung of the comic ray nuclei and electrons.
}

to dominate (or even be the only one) in the most dense and cold GMCs.

Galactic CRs are thought to be accelerated by shocks from SNRs or even Wolf-Rayet stars (e.g., Kotera \& Olinto 2011) and have a fairly homogenous distribution throughout the Milky Way, decreasing with galactocentric distance (e.g., Bloemen 1989). It is conceivable that the production of CRs is significantly increased above the solar value in the presence of massive star formation. It has been shown for the nearby starburst galaxy NGC 253 that enhanced CR emission originating from the massive star formation in the nucleus with $\mathrm{SFR} \sim 0.1 M_{\odot} \mathrm{yr}^{-1}$ can explain the observed substantial heating of the molecular gas (Bradford et al. 2003; Hailey-Dunsheath et al. 2008); a similar scenario has been proposed by Papadopoulos (2010) to explain the $\mathrm{CO}$ emission in star forming ULIRGs. These regions are called CR-dominated regions (CRDRs) and might have a distinct chemical signature (Bayet et al. 2011).

\section{A.1.2. $\mathrm{H}_{2}$ Formation Heating}

The formation of $\mathrm{H}_{2}$ on grains (e.g., Hollenbach \& Salpeter 1971) also provides heating of the gas, as some fraction of the released binding energy goes into kinetic energy and thus subsequently heats the gas (Goldsmith \& Langer 1978). The heating rate is proportional to the total hydrogen density (in molecular and atomic form) in quadrature times the fractional abundance of atomic hydrogen. Goldsmith \& Langer (1978) note that if $\mathrm{H}_{2}$ destruction by CRs (see above) is taken into account, the maximum heating rate from $\mathrm{H}_{2}$ formation could be $1 / 3$ of the CR heating rate, however, in reality, the steadystate assumption might not be fully correct. Additionally, the exact properties of dust (grain sizes, PAHs, temperature, etc.) might affect the $\mathrm{H}_{2}$ formation rates, especially in diffuse, i.e., low density, gas (Wolfire et al. 2008).

\section{A.1.3. Dust Heating}

The predominant heating mechanism for dust (in molecular clouds) is via the photo-electric effect (Draine 1978). As dust couples better to the ISRF than the molecular gas, the dust kinetic temperature $T_{\text {dust }}$ is typically larger than the gas kinetic temperature $T_{\text {gas }}$ within a molecular cloud (Maloney \& Black 1988). Thus, collisions of $\mathrm{H}_{2}$ with dust grains could increase the kinetic energy of the gas. As the dust heating is governed by the available radiation field, it is expected that increasing the local ISFR by adding an intense radiation field from, e.g., young (OB) stars or an AGN, will lead to higher dust and thus potentially higher gas temperature if both are well mixed and coupled.

The energy transfer between dust and gas via collisions can be described by the energy difference of the $\mathrm{H}_{2}$ molecule before and after a collision with a dust grain (Burke \& Hollenbach 1983). As it is possible that the dust temperature $T_{\text {dust }}$ is higher than the gas temperature $T_{\mathrm{gas}}$, as well as the other way around, both heating or cooling of the gas is possible via this mechanism. The heating/cooling rate is proportional to $n\left(\mathrm{H}_{2}\right)^{2} \times \Delta T \times T_{\mathrm{gas}}^{0.5}$ with $\Delta T=T_{\text {gas }}-T_{\text {dust }}$ (Goldsmith 2001).

The radiation from the diffuse UV-visible-IR ISRF will heat dust grains to temperatures higher than the gas temperatures observed for the dense cores of dark clouds, however, for dust grains inside the clouds, this ISRF will be attenuated. Goldsmith (2001) calculates that in order to achieve dust temperatures of $6-10 \mathrm{~K}$, a visual extinction to the surface of the cloud of 10-12 mag is required, consistent with observations (Kramer et al. 1999). He notes that a further drop in dust temperature is not expected due to heating provided by the re-emission from 
dust grains themselves. Thus, in molecular clouds (without star formation) heating by dust is proportional to the attenuated ISFR times the $\mathrm{H}_{2}$ density $n\left(\mathrm{H}_{2}\right)$ divided by the gas-to-dust ratio. At the same time, the dust cools with $T_{\text {dust }}^{6}$.

Thus significant heating from dust is only expected to occur at high $\mathrm{H}_{2}$ densities $n\left(\mathrm{H}_{2}\right)$ above $10^{4} \mathrm{~cm}^{-3}$ where the coupling of gas and dust via gas-dust collisions is strong and can significantly reduce the gas temperature while only slightly increasing the dust temperature (Goldsmith 2001). Examples might be dense clouds close to or with embedded star forming regions (with OB stars) as such clouds have elevated temperatures of 40-80 K (e.g., Goldsmith \& Langer 1978). Recent simulations confirm this picture (e.g., Juvela \& Ysard 2011), but the authors caution that a varying grain size distribution within clouds can affect the gas-dust coupling and thus the resulting gas temperature by $1-2 \mathrm{~K}$ in dense core.

\section{A.1.4. UV Heating}

It is expected that the intense (UV) radiation from young stars in star forming regions heats the gas. Two mechanisms are generally considered: heating via the grain photoelectric effect (Tielens \& de Jong 1979) and direct UV pumping of $\mathrm{H}_{2}$ molecules followed by collisional de-excitation (Hollenbach \& McKee 1979; Black \& Dalgarno 1976; Sternberg \& Dalgarno 1989). Detailed models of these PDRs (Tielens \& Hollenbach 1985) have been derived where UV photons penetrate through the surface into the cloud while being attenuated and produce layers of ionized, atomic, and warm molecular gas around the cold molecular gas. How far a UV photon can penetrate into a cloud depends on the dust opacity.

Gas heating via the photoelectric effect has been shown to occur by PAH molecules and small graphite grains (grains with size $a<100 \AA$; Bakes \& Tielens 1994). About $50 \%$ of the UV photon energy is absorbed by these small dust particles. Absorption of a FUV photon by such a dust grain may lead to ionization and thus the ejection of an electron. The excited electron loses energy through inelastic collisions with carbon atoms inside the grain on its way to the surface and will leave the grain with some kinetic energy. Via inelastic collisions with gas molecules, the thermal energy of the gas will be raised (e.g., Tielens \& Hollenbach 1985; Bakes \& Tielens 1994). The photoelectric effect on interstellar dust grains should dominate the heating of the neutral atomic ISM and play an important role in the warm inter-cloud medium and in PDR regions of cold molecular clouds (i.e., UV illuminated regions such as $\mathrm{H} \mathrm{II}$ regions, planetary nebulae, etc.). The heating efficiency could be up to 3\% (for neutral grains; lower in grains that are charged).

Dust plays an important role in attenuating the UV radiation (at optical depths $A_{V} \sim 1$ ) that controls gas heating in order to prevent higher gas temperatures (Maloney \& Black 1988). For a simple slab geometry of the molecular gas, the grain photoelectric heating is only important in the outermost layer of a molecular cloud in the presence of radiation from the ISRF (dominated by old stellar populations); its contribution could become significant in the case of intense radiation fields from young stars (e.g., for the dense clouds in the center of M82; Maloney \& Black 1988). At low column densities, which are present at the outer surface of molecular clouds, photoionization heating can become important. A comparison of contemporary PDR codes summarizing the main characteristics deemed important is presented by Röllig et al. (2007).

\section{A.1.5. X-Ray Heating}

X-ray dominated regions (XDRs) are expected to be present close to nearby AGNs where the strong X-ray radiation from the accreting black hole impinges on surrounding molecular clouds. A recent model of XDRs is presented by Meijerink et al. (2006). While XDR modeling is analogous to PDR models, it is interesting to highlight some profound differences: the assumed input spectrum is much harder for an AGN than for hot, e.g., OB, stars and the main heating source is direct photoionization of the gas, which produces fast electrons. These electrons collisionally excite the $\mathrm{H}$ and $\mathrm{H}_{2}$ gas, which emits Ly $\alpha$ and Lyman-Werner photons that ionize atoms and ionize and dissociate molecules (e.g., $\mathrm{H}_{2}$ and $\mathrm{CO}$; Maloney et al. 1996). As hard X-rays can penetrate deep into dense clouds, the heating efficiency in XDRs is close to unity, however, the $\mathrm{X}$-ray heating and ionization rates depend on the actual slope of the X-ray spectrum (Meijerink et al. 2006).

In this context, it is interesting to note that Lepp \& McCray (1983) were among the first to consider the impact of a X-ray source inside a molecular cloud, as most stars are sources of soft $\mathrm{X}$-ray emission and number of accreting white dwarfs should be present in each GMC. While the contribution from X-ray heating via the old stellar population is hard to assess separately from CR heating, Glassgold et al. (2012) revisited the impact of CR and X-ray heating on cold dense clouds based on new findings of the $\mathrm{H}_{3}^{+}$ion abundance in Galactic clouds. They find that the combined CR and X-ray heating (also referred to as chemical heating) significantly changes with the properties of the medium (molecular/electron fraction, total density of $\mathrm{H}$ nuclei) but has only a weak temperature dependence.

\section{A.2. Carbon-monoxide (CO) Line Emission}

It is generally assumed that the $\mathrm{H}_{2}$ and $\mathrm{CO}$ molecular gas is in thermal equilibrium so that the kinetic temperatures are the same. Detailed calculations for dense, fully shielded molecular regions (i.e., $A_{V} \geqslant 10$ ) assuming a steady-state chemical composition predict that the $\mathrm{CO}$ molecules dominate the cooling in the low-density $\left(n\left(\mathrm{H}_{2}\right)<10^{5} \mathrm{~cm}^{-3}\right)$ and low-temperature $\left(T_{\text {kin }} \leqslant 40 \mathrm{~K}\right)$ regime while other molecules become the dominant coolants at high densities and high temperatures (Neufeld et al. 1995).

The radial distribution of $\mathrm{H}_{2}$ and $\mathrm{CO}$ molecules across the surface into the center of molecular clouds varies as the dissociation conditions of $\mathrm{CO}$ and $\mathrm{H}_{2}$ are not the same. van Dishoeck \& Black (1988) provide a detailed description and modeling of CO photodissociation. While the unattenuated photodissociation from stellar UV light for CO is high, substantial reductions happen inside the clouds, including: (1) self-shielding, i.e., growth toward saturation of the absorption in the line itself with increasing depth and column density; (2) mutual shielding, i.e., the blending of lines of the isotopic species with those in the same band of the more abundant ${ }^{12} \mathrm{CO}$ molecule; (3) shielding by coincident lines of $\mathrm{H}$ and $\mathrm{H}_{2}$; and (4) attenuation of radiation by dust particles and carbon atoms, which significantly decreases the photodissociation of $\mathrm{CO}$ even inside diffuse clouds. Recently, Visser et al. (2009) updated the calculations from van Dishoeck \& Black (1988) using the latest laboratory data and fully treated the heavier $\mathrm{CO}$ isotopologues ${ }^{13} \mathrm{CO}, \mathrm{C}^{18} \mathrm{O}$, and ${ }^{13} \mathrm{C}^{17} \mathrm{O}$. In addition, they extended the calculations to higher excitation temperatures, as well as densities covering PDR and circumstellar disk environments. 
However, the exact relation between $\mathrm{CO}$ line emission (in particular, the low-J transition) and $\mathrm{H}_{2}$ gas properties is still not very well understood. Recently, Glover et al. (2010) modeled the $\mathrm{CO}$ formation. They found that both molecular species are expected to form quickly, however the relationship between $\mathrm{CO}$ abundance and gas density is more complex. The $\mathrm{CO}$ abundance primarily depends on the photodissociation rate rather than the gas density (Glover \& Low 2011). Clark et al. (2012) investigate the timescales required for a molecular cloud to become $\mathrm{CO}$ detectable starting with a pure atomic phase in a turbulent medium. They find that the timescales depend critical on the conditions of the medium out of which the cloud forms with $\mathrm{CO}$ typically being only detectable about $\sim 2$ Myr before the onset of star formation. As the neutral gas disk of M51 covered by the PAWS observations is molecular dominated (e.g., Schuster et al. 2007), it is unclear how to adapt these modeling results to the specific case of M51.

\section{A.3. The Relation between CO Line Emission and Molecular Hydrogen Mass}

In order to be able to relate the observed $\mathrm{CO}$ line emission to the amount of $\mathrm{H}_{2}$ mass present, one needs to understand how the molecular gas is cooling. As the type of main coolants changes with temperature, gas density, and relative abundance of the coolants (which themselves depend on the ionization state of the gas and the intrinsic metallicity of the gas, as well as other chemical processes), the observed $\mathrm{CO}$ emission per $\mathrm{H}_{2}$ molecule is expected to vary as well (e.g., Goldsmith \& Langer 1978). Typically, one expects the following type of coolants (ignoring metallicity effects): the cold (temperatures of $10-40 \mathrm{~K}$ or below $100 \mathrm{~K}$ ) molecular gas cools via atomic and molecular line emission, however, the main cooling lines shift from atomic lines such as $\mathrm{C}$, $\mathrm{O}$ I, etc. in the low density regime $\left(n\left(\mathrm{H}_{2}\right)<10^{3} \mathrm{~cm}^{-3}\right)$ to $\mathrm{CO}$ as the main cooling channel (for $\left.n\left(\mathrm{H}_{2}\right)<3 \times 10^{4} \mathrm{~cm}^{-3}\right)$ and a wide range of species including water, hydrides, molecular ions, etc. for the highest densities where ${ }^{12}$ CO can freeze out (Goldsmith \& Langer 1978).

In addition to the physical heating and cooling conditions of the molecular gas described above, the abundance of $\mathrm{CO}$ to $\mathrm{H}_{2}$ molecules becomes crucial when determining the relation between $\mathrm{CO}$ line emission and $\mathrm{H}_{2}$ mass present. Metallicity has the most profound effect on the abundance ratio, while other effects such as $\mathrm{CO}$ freeze-out for very high density gas or shocks could also alter the chemical composition of the molecular gas (Goldsmith 2001; Meijerink et al. 2010; Bayet et al. 2011). However, it is anticipated that metallicity has a fairly uniform effect while the other properties that have been discussed are expected to be much more localized across a galaxy.

For the case of average molecular clouds, Maloney \& Black (1988) showed that the conversion factor $X_{\mathrm{CO}}$ between $\mathrm{CO}$ intensity and $N\left(\mathrm{H}_{2}\right)$ column density depends on the kinetic temperature $T_{\text {kin }}$ and velocity dispersion of the molecular cloud $\sigma_{c}$, which in turn is related to the $\mathrm{H}_{2}$ density such that $X_{\mathrm{CO}} \propto$ $T_{\mathrm{kin}}^{-1} \sigma_{\mathrm{c}} \sim T_{\mathrm{kin}}^{-1} \sqrt{n\left(\mathrm{H}_{2}\right)}$ (for a slightly different dependence, see Shetty et al. 2011). This dependency can be easily understood as the line intensity being directly proportional to the excitation temperature (i.e., $I_{\mathrm{CO}} \sim T_{\mathrm{ex}}$ ) in optically thick clouds (where $T_{\mathrm{ex}} \sim T_{\text {kin }}$ is valid, especially at the lower transitions), thus the ratio $I_{\mathrm{CO}} / T_{\text {kin }}$ is basically constant. For a gravitationally bound cloud, the velocity dispersion is proportional to $\sqrt{M}$, with the cloud mass $M$ being proportional to the $n\left(\mathrm{H}_{2}\right)$ density for a homogenous cloud (see Dickman et al. 1986, who showed that this assumption is also correct for ensembles of clouds).
The abundance of $\mathrm{CO}$ relative to $\mathrm{H}_{2}$ is another critical parameter in determining the $X_{\mathrm{CO}}$ factor. If the metallicity is smaller, then the dust abundance is smaller, thus the attenuation of the UV radiation with depth into the cloud is smaller. While $\mathrm{H}_{2}$ self-shielding will prevent a drastic reduction of the $\mathrm{H}_{2}$ zone, the destruction of $\mathrm{CO}$ will lead to an increase in cloud temperature (Maloney \& Black 1988). The importance of this effect will depend on the column density. The implication is that a decreased $\mathrm{CO}$ abundance will cause the $\mathrm{CO}$ line to be no longer optically thick except at the cloud cores for clouds with similar column density but different $\mathrm{CO}$ abundance (e.g., only $\mathrm{CO}$ emission from the core of LMC clouds versus the full cloud for Milky Way clouds). For more details on the latest observational findings on the conversion factor in external galaxies, we refer the reader to Sandstrom et al. (2013) and Leroy et al. (2013).

Based on the continuous conversion factor of $X_{\mathrm{CO}}=$ $\left(\min \left[4675 \times I_{\mathrm{CO}}^{-0.32}\right] \times 10^{20}\right) / Z^{\prime-.65}$ derived by Narayanan et al. (2012), we expect a range of $(1-3) \times 10^{20} \mathrm{~cm}^{-2}\left(\mathrm{~K} \mathrm{~km} \mathrm{~s}^{-1}\right)^{-1}$ for our measured $I_{\mathrm{CO}}$ per pixel, assuming solar metallicity, as observed for the PAWS FoV (Bresolin et al. 2004; Moustakas et al. 2010), i.e., $Z^{\prime}=1$. This is similar to the results of Schinnerer et al. (2010) using a line velocity gradient analysis and the virial mass measurements of individual GMCs by Colombo et al. (2013a).

\section{APPENDIX B}

\section{CALCULATING ANGULAR OFFSETS VIA POLAR CROSS-CORRELATION}

In order to quantify the radial variations between two different tracers along the spiral arms, we employ a polar cc (see Figures 13 and 14). This method allows for easy identification of variation in the position of the maximum correlation as a function of radius.

We calculate the angular offset $\phi$ between the molecular gas spiral arms traced in CO and ISM, stellar, and star formation tracers at a second wavelength $\lambda$ via convolution of the two corresponding maps. All images have the same astrometry, angular resolution, and pixel sampling (as described in Section 3). Prior to the convolution, the images were deprojected from the sky plane into the galaxy plane using an inclination of $i=21^{\circ}$ and $\mathrm{PA}=173^{\circ}$ (as determined from analysis of the PAWS velocity field; Colombo et al. 2013b). Next, the images were projected onto a polar coordinate system (see Section 4.2, e.g., Figure 4) and placed into the spiral arm frame assuming a logarithmic spiral arm pitch angle of $i_{p}=21^{\circ}$. The convolution is performed in log-polar coordinates. The spiral arm frame has been chosen for ease of presentation as the maximum of the auto correlation should occur very near the same azimuthal position in each radial bin.

As our measure of the angular offset $\phi$ between the ridge of the spiral arm traced by $\mathrm{CO}$ and the second tracer at wavelength $\lambda$, we adopt the intensity-weighted first moment of the cc profile tracing the location of maximum correlation. This is in contrast to using polynomial fits to the profile or using the maximum itself. We prefer this approach for several reasons. Moment measurements made directly from the cc profile are less susceptible to systematic uncertainties introduced by, e.g., the choice of the order of or limits of the polynomial fits to the profile, which typically limit the reliability of the measured offset (Foyle et al. 2010). The first moment, in particular, is preferred for its reduced sensitivity (compared with the 
maximum) to local variations in the flux tracing structure at spatial scales smaller than the arm width (e.g., brightness peaks in $\mathrm{CO}$ emission tracing individual clouds). ${ }^{20}$

From the second moment, we can obtain a measure of the intrinsic thickness of the arms. For arms with azimuthal intensity profiles that are roughly Gaussian, the convolved profile has a measured width of $\sigma=\sqrt{\sigma_{\mathrm{CO}}^{2}+\sigma_{\lambda}^{2}}$, where $\sigma_{\mathrm{CO}}$ and $\sigma_{\lambda}$ are the intrinsic widths of the arms traced by $\mathrm{CO}$ emission and at wavelength $\lambda$, respectively. We verified that the width at $95 \%$ maximum of the CO-brightness auto-correlation profile (CO crossed with itself) corresponds well with the width estimated by eye from the morphology of CO brightness. We therefore measure the convolved arm width as $w \sim 0.64 \sigma$, where $\sigma$ is the second moment of the profile. We adopt this width as the uncertainty on our measurement of $\phi$ (see Figures 5 and 11).

\section{REFERENCES}

Adler, D. S., Lo, K. Y., \& Allen, R. J. 1991, ApJ, 382, 475

Allen, R. J. 1992, ApJ, 399, 573

André, P., Men'shchikov, A., Bontemps, S., et al. 2010, A\&A, 518, L102

Aniano, G., Draine, B. T., Calzetti, D., et al. 2012, ApJ, 756, 138

Aniano, G., Draine, B. T., Gordon, K. D., \& Sandstrom, K. 2011, PASP, 123,1218

Bakes, E. L. O., \& Tielens, A. G. G. M. 1994, ApJ, 427, 822

Bastian, N., Gieles, M., Efremov, Y. N., \& Lamers, H. J. G. L. M. 2005, A\&A, 443, 79

Bayet, E., Williams, D. A., Hartquist, T. W., \& Viti, S. 2011, MNRAS, 414, 1583

Beckman, J. E., Peletier, R. F., Knapen, J. H., Corradi, R. L. M., \& Gentet, L. J. 1996, ApJ, 467, 175

Berkhuijsen, E. M., Bajaja, E., \& Beck, R. 1993, A\&A, 279, 359

Bernard, J.-P., Reach, W. T., Paradis, D., et al. 2008, AJ, 136, 919

Bigiel, F., Leroy, A., Seibert, M., et al. 2010, ApJL, 720, L31

Bigiel, F., Leroy, A., Walter, F., et al. 2008, AJ, 136, 2846

Bigiel, F., Leroy, A. K., Walter, F., et al. 2011, ApJL, 730, L13

Black, J. H., \& Dalgarno, A. 1976, ApJ, 203, 132

Blitz, L., \& Williams, J. P. 1999, arXiv:astro-ph/9903382

Bloemen, H. 1989, ARA\&A, 27, 469

Bloemen, J. B. G. M., Strong, A. W., Mayer-Hasselwander, H. A., et al. 1986, A\&A, 154, 25

Bohlin, R. C., Savage, B. D., \& Drake, J. F. 1978, ApJ, 224, 132

Bolatto, A. D., Leroy, A. K., Rosolowsky, E., Walter, F., \& Blitz, L. 2008, ApJ, 686, 948

Bradford, C. M., Nikola, T., Stacey, G. J., et al. 2003, ApJ, 586, 891

Brauher, J. R., Dale, D. A., \& Helou, G. 2008, ApJS, 178, 280

Bresolin, F., Garnett, D. R., \& Kennicutt, R. C., Jr. 2004, ApJ, 615, 228

Brunner, G., Sheth, K., Armus, L., et al. 2008, ApJ, 675, 316

Burke, J. R., \& Hollenbach, D. J. 1983, ApJ, 265, 223

Calzetti, D., Kennicutt, R. C., Jr., Bianchi, L., et al. 2005, ApJ, 633, 871

Chandar, R., Whitmore, B. C., Calzetti, D., et al. 2011, ApJ, 727, 88

Cho, J., \& Vishniac, E. T. 2000, ApJ, 538, 217

Christensen, C., Quinn, T., Governato, F., et al. 2012, MNRAS, 425, 3058

Ciardullo, R., Feldmeier, J. J., Jacoby, G. H., et al. 2002, ApJ, 577, 31

Clark, P. C., Glover, S. C. O., Klessen, R. S., \& Bonnell, I. A. 2012, MNRAS, 424, 2599

Colombo, D., Schinnerer, E., Hughes, A., et al. 2013a, ApJ, submitted

Colombo, D., Schinnerer, E., Meidt, S. E., et al. 2013b, ApJ, submitted

Condon, J. J. 1992, ARA\&A, 30, 575

Crane, P. C., \& van der Hulst, J. M. 1992, AJ, 103, 1146

Croxall, K. V., Smith, J. D., Wolfire, M. G., et al. 2012, ApJ, 747, 81

Dale, D. A., Aniano, G., Engelbracht, C. W., et al. 2012, ApJ, 745, 95

Devereux, N. A., \& Young, J. S. 1990, ApJ, 359, 42

Dickman, R. L. 1978, ApJS, 37, 407

Dickman, R. L., Snell, R. L., \& Schloerb, F. P. 1986, ApJ, 309, 326

Donovan Meyer, J., Koda, J., Momose, R., et al. 2011, ApJ, 744, 42

Draine, B. T. 1978, ApJS, 36, 595

\footnotetext{
${ }^{20}$ We note that compared with the maximum, the first moment also conveys additional information regarding the symmetry of a given arm, or how the emission is distributed relative to the spiral ridge; asymmetric arms at one or both wavelengths result in skewed profiles. This skewness can also be measured directly from the profile as the third moment.
}

Draine, B. T., \& Li, A. 2007, ApJ, 657, 810

Dumas, G., Schinnerer, E., Tabatabaei, F. S., et al. 2011, AJ, 141, 41 Elmegreen, B. G., Seiden, P. E., \& Elmegreen, D. M. 1989, ApJ, 343, 602 Espada, D., Komugi, S., Muller, E., et al. 2012, ApJL, 760, L25

Fiebig, D., \& Guesten, R. 1989, A\&A, 214, 333

Fisher, D. B., \& Drory, N. 2010, ApJ, 716, 942

Fletcher, A., Beck, R., Shukurov, A., Berkhuijsen, E. M., \& Horellou, C. 2011, MNRAS, 412, 2396

Ford, H. C., Crane, P. C., Jacoby, G. H., Lawrie, D. G., \& van der Hulst, J. M. 1985, ApJ, 293, 132

Foyle, K., Rix, H.-W., Dobbs, C. L., Leroy, A. K., \& Walter, F. 2011, ApJ, 735,101

Foyle, K., Rix, H.-W., Walter, F., \& Leroy, A. K. 2010, ApJ, 725, 534

Garcia-Burillo, S., Guélin, M., \& Cernicharo, J. 1993, A\&A, 274, 123

Glassgold, A. E., Galli, D., \& Padovani, M. 2012, ApJ, 756, 157

Glover, S. C. O., Federrath, C., Mac Low, M. M., \& Klessen, R. S 2010, MNRAS, 404, 2

Glover, S. C. O., \& Low, M. M. M. 2011, MNRAS, 412, 337

Gnedin, N. Y., Tassis, K., \& Kravtsov, A. V. 2009, ApJ, 697, 55

Goldsmith, P. F. 2001, ApJ, 557, 736

Goldsmith, P. F., \& Langer, W. D. 1978, ApJ, 222, 881

Gracia-Carpio, J., Sturm, E., Hailey-Dunsheath, S., et al. 2011, ApJL, 728, L7

Gratier, P., Braine, J., Rodriguez-Fernandez, N. J., et al. 2012, A\&A, 542, A108

Groves, B., Krause, O., Sandstrom, K., et al. 2012, MNRAS, 426, 892

Groves, B. A., Cho, J., Dopita, M., \& Lazarian, A. 2003, PASA, 20, 252

Gutiérrez, L., Beckman, J. E., \& Buenrostro, V. 2011, AJ, 141, 113

Haas, M. R., Gieles, M., Scheepmaker, R. A., Larsen, S. S., \& Lamers, H. J. G. L. M. 2008, A\&A, 487, 937

Hagiwara, Y. 2007, AJ, 133, 1176

Hailey-Dunsheath, S., Nikola, T., Stacey, G. J., et al. 2008, ApJL, 689, L109

Heiderman, A., Evans, N. J., Allen, L. E., Huard, T., \& Heyer, M. 2010, ApJ, 723,1019

Heitsch, F., Whitney, B. A., Indebetouw, R., et al. 2007, ApJ, 656, 227

Helfer, T. T., \& Blitz, L. 1997, ApJ, 478, 162

Helfer, T. T., Thornley, M. D., Regan, M. W., et al. 2003, ApJS, 145, 259

Hennebelle, P., \& Audit, E. 2007, A\&A, 465, 431

Hennebelle, P., \& Audit, E. 2008, EAS, 31, 15

Henry, A. L., Quillen, A. C., \& Gutermuth, R. 2003, AJ, 126, 2831

Hirota, A., Kuno, N., Sato, N., et al. 2011, ApJ, 737, 40

Hoernes, P., Berkhuijsen, E. M., \& Xu, C. 1998, A\&A, 334, 57

Hollenbach, D., \& McKee, C. F. 1979, ApJS, 41, 555

Hollenbach, D., \& Salpeter, E. E. 1971, ApJ, 163, 155

Hopkins, P. F., Murray, N., Quataert, E., \& Thompson, T. A. 2010, MNRAS, 401, L19

Hughes, A., Meidt, S. E., Colombo, D., et al. 2013a, ApJ, in press (arXiv: 1309.3453)

Hughes, A., Meidt, S. E., Schinnerer, E., et al. 2013b, ApJ, in press (arXiv:1304.1209)

Hwang, N., \& Lee, M. G. 2008, AJ, 135, 1567

Israel, F., \& Rowan-Robinson, M. 1984, ApJ, 283, 81

Juvela, M., \& Ysard, N. 2011, ApJ, 739, 63

Karim, A., Schinnerer, E., Martinez-Sansigre, A., et al. 2011, ApJ, 730, 61

Kennicutt, R. C., Calzetti, D., Aniano, G., et al. 2011, PASP, 123, 1347

Kennicutt, R. C., Jr. 1998, ApJ, 498, 541

Kennicutt, R. C., Jr., Armus, L., Bendo, G., et al. 2003, PASP, 115, 928

Kennicutt, R. C. J. 1989, ApJ, 344, 685

Ko, C. M., \& Parker, E. N. 1989, ApJ, 341, 828

Koda, J., Scoville, N., Sawada, T., et al. 2009, ApJL, 700, L132

Kohno, K., Kawabe, R., Tosaki, T., \& Okumura, S. K. 1996, ApJL, 461, L29

Kotera, K., \& Olinto, A. V. 2011, ARA\&A, 49, 119

Kramer, C., Alves, J., Lada, C. J., et al. 1999, A\&A, 342, 257

La Vigne, M. A., Vogel, S. N., \& Ostriker, E. C. 2006, ApJ, 650, 818

Lada, C. J., Alves, J. F., \& Lombardi, M. 2007, Protostars and Planets V (Tucson, AZ: Univ. Arizona Press), 3

Lamers, H. J. G. L. M., Panagia, N., Scuderi, S., et al. 2002, ApJ, 566, 818

Lee, J. H., Hwang, N., \& Lee, M. G. 2011, ApJ, 735, 75

Lepp, S., \& McCray, R. 1983, ApJ, 269, 560

Leroy, A., Bolatto, A. D., Simon, J. D., \& Blitz, L. 2005, ApJ, 625, 763

Leroy, A. K., Bigiel, F., de Blok, W. J. G., et al. 2012, AJ, 144, 3

Leroy, A. K., Walter, F., Brinks, E., et al. 2008, AJ, 136, 2782

Leroy, A. K., Walter, F., Sandstrom, K., et al. 2013, AJ, 146, 19

Lisenfeld, U., \& Völk, H. J. 2000, A\&A, 354, 423

Liu, F., \& Gao, Y. 2010, ApJ, 713, 524

Lombardi, M., Alves, J., \& Lada, C. J. 2006, A\&A, 454, 781

Louie, M., Koda, J., \& Egusa, F. 2013, ApJ, 763, 94

Maddox, L. A., Cowan, J. J., Kilgard, R. E., Schinnerer, E., \& Stockdale, C. J. 2007, AJ, 133, 2559 
Maloney, P., \& Black, J. H. 1988, ApJ, 325, 389

Maloney, P. R., Hollenbach, D. J., \& Tielens, A. G. G. M. 1996, ApJ, 466, 561

Marscher, A. P., \& Brown, R. L. 1978, ApJ, 221, 588

Matsushita, S., Muller, S., \& Lim, J. 2007, A\&A, 468, L49

Matsushita, S., Sakamoto, K., Kuo, C.-Y., et al. 2004, ApJL, 616, L55

Mead, K. N., Kutner, M. L., \& Evans, N. J. I. 1990, ApJ, 354, 492

Meidt, S. E., Rand, R. J., Merrifield, M. R., Shetty, R., \& Vogel, S. N. 2008, ApJ, 688,224

Meidt, S. E., Schinnerer, E., García-Burillo, S., et al. 2013, ApJ, in press (arXiv:1304.7910)

Meidt, S. E., Schinnerer, E., Knapen, J. H., et al. 2012, ApJ, 744, 17

Meier, D. S., \& Turner, J. L. 2005, ApJ, 618, 259

Meier, D. S., \& Turner, J. L. 2012, ApJ, 755, 104

Meijerink, R., Spaans, M., \& Israel, F. P. 2006, ApJL, 650, L103

Meijerink, R., Spaans, M., Loenen, A. F., \& van der Werf, P. P. 2010, A\&A, 525, A119

Menéndez-Delmestre, K., Sheth, K., Schinnerer, E., Jarrett, T. H., \& Scoville, N. Z. 2007, ApJ, 657, 790

Mentuch Cooper, E., Wilson, C. D., Foyle, K., et al. 2012, ApJ, 755, 165

Mink, D. J. 1999, in ASP Conf. Ser. 172, Astronomical Data Analysis Software and Systems VIII, ed. D. M. Mehringer, R. L. Plante, \& D. A. Roberts (San Francisco, CA: ASP), 498

Mooney, T. J., \& Solomon, P. M. 1988, ApJL, 334, L51

Morrissey, P., Schiminovich, D., Barlow, T. A., et al. 2005, ApJL, 619, L7

Moustakas, J., Kennicutt, R. C., Tremonti, C. A., et al. 2010, ApJS, 190, 233

Muñoz-Mateos, J. C., Boissier, S., Gil de Paz, A., et al. 2011, ApJ, 731, 10

Muñoz-Mateos, J. C., Gil de Paz, A., Boissier, S., et al. 2009, ApJ, 701, 1965

Murgia, M., Crapsi, A., Moscadelli, L., \& Gregorini, L. 2002, A\&A, 385, 412

Murgia, M., Helfer, T. T., Ekers, R., et al. 2005, A\&A, 437, 389

Murphy, E. J., Bremseth, J., Mason, B. S., et al. 2012, ApJ, 761, 97

Murphy, E. J., Condon, J. J., Schinnerer, E., et al. 2011, ApJ, 737, 67

Mutchler, M., Beckwith, S. V. W., Bond, H., et al. 2005, BAAS, 37, 452

Narayanan, D., Krumholz, M. R., Ostriker, E. C., \& Hernquist, L. 2012, MNRAS, 421, 3127

Neufeld, D. A., Lepp, S., \& Melnick, G. J. 1995, ApJS, 100, 132

Nieten, C., Neininger, N., Guélin, M., et al. 2006, A\&A, 453, 459

Niklas, S., \& Beck, R. 1997, A\&A, 320, 54

Onodera, S., Kuno, N., Tosaki, T., et al. 2010, ApJL, 722, L127

Ostriker, E. C., Stone, J. M., \& Gammie, C. F. 2001, ApJ, 546, 980

Paladino, R., Murgia, M., Helfer, T. T., et al. 2006, A\&A, 456, 847

Papadopoulos, P. P. 2010, ApJ, 720, 226

Parkin, T., Wilson, C. D., Schirm, M. R. P., et al. 2013, ApJ, 776, 65

Patrikeev, I., Fletcher, A., Stepanov, R., et al. 2006, A\&A, 458, 441

Pety, J. 2005, in SF2A-2005: Semaine de l'Astrophysique Francaise, ed. F. Casoli, T. Contini, J. M. Hameury, \& L. Pagani (Les Ulis Cedex A: EdPSciences), 721

Pety, J., \& Falgarone, É. 2000, A\&A, 356, 279

Pety, J., Schinnerer, E., Leroy, A. K., et al. 2013, ApJ, in press (arXiv:1304.1396)

Pineda, J. E., Caselli, P., \& Goodman, A. A. 2008, ApJ, 679, 481

Protheroe, R. J., Ott, J., Ekers, R. D., Jones, D. I., \& Crocker, R. M. 2008, MNRAS, 390, 683

Rebolledo, D., Wong, T., Leroy, A., Koda, J., \& Meyer, J. D. 2012, ApJ, 757,155

Rickard, L. J., Turner, B. E., Palmer, P., Morris, M., \& Zuckerman, B. 1977, ApJ, 213,673

Rix, H.-W., \& Rieke, M. J. 1993, ApJ, 418, 123

Robertson, B. E., \& Kravtsov, A. V. 2008, ApJ, 680, 1083

Röllig, M., Abel, N. P., Bell, T., et al. 2007, A\&A, 467, 187
Rosolowsky, E., Engargiola, G., Plambeck, R., \& Blitz, L. 2003, ApJ, 599, 258

Roussel, H. 2013, PASP, 125, 1126

Sanders, D. B., Scoville, N. Z., \& Solomon, P. M. 1985, ApJ, 289, 373

Sandstrom, K. M., Bolatto, A. D., Draine, B. T., Bot, C., \& Stanimirović, S 2010, ApJ, 715, 701

Sandstrom, K. M., Leroy, A. K., Walter, F., et al. 2013, ApJ, 777, 5

Scheepmaker, R. A., Haas, M. R., Gieles, M., et al. 2007, A\&A, 469, 925

Scheepmaker, R. A., Lamers, H. J. G. L. M., Anders, P., \& Larsen, S. S. 2009, A\&A, 494, 81

Schinnerer, E., Weiss, A., Aalto, S., \& Scoville, N. Z. 2010, ApJ, 719, 1588

Schruba, A., Leroy, A. K., Walter, F., Sandstrom, K., \& Rosolowsky, E. 2010, ApJ, 722, 1699

Schruba, A., Leroy, A. K., Walter, F., et al. 2011, AJ, 142, 37

Schuster, K. F., Kramer, C., Hitschfeld, M., Garcia-Burillo, S., \& Mookerjea, B. 2007, A\&A, 461, 143

Scoville, N. Z., Polletta, M., Ewald, S., et al. 2001, AJ, 122, 3017

Scoville, N. Z., Yun, M. S., Armus, L., \& Ford, H. 1998, ApJL, 493, L63

Sheth, K., Regan, M., Hinz, J. L., et al. 2010, PASP, 122, 1397

Sheth, K., Vogel, S. N., Regan, M. W., et al. 2002, AJ, 124, 2581

Shetty, R., Glover, S. C., Dullemond, C. P., et al. 2011, MNRAS, 415, 3253

Shetty, R., Vogel, S. N., Ostriker, E. C., \& Teuben, P. J. 2007, ApJ, 665, 1138

Smith, J. D. T., Draine, B. T., Dale, D. A., et al. 2007, ApJ, 656, 770

Solomon, P. M., Rivolo, A. R., Barrett, J., \& Yahil, A. 1987, ApJ, 319, 730

Stacey, G. J., Geis, N., Genzel, R., et al. 1991, ApJ, 373, 423

Stacey, G. J., Hailey-Dunsheath, S., Ferkinhoff, C., et al. 2010, ApJ, 724, 957

Sternberg, A., \& Dalgarno, A. 1989, ApJ, 338, 197

Suchkov, A., Allen, R. J., \& Heckman, T. M. 1993, ApJ, 413, 542

Tabatabaei, F. S., Beck, R., Krügel, E., et al. 2007, A\&A, 475, 133

Terashima, Y., \& Wilson, A. S. 2001, ApJ, 560, 139

Thompson, T. A., Quataert, E., \& Waxman, E. 2007, ApJ, 654, 219

Tielens, A. G. G. M. 2008, ARA\&A, 46, 289

Tielens, A. G. G. M., \& de Jong, T. 1979, A\&A, 75, 326

Tielens, A. G. G. M., \& Hollenbach, D. 1985, ApJ, 291, 722

Tilanus, R. P. J., Allen, R. J., van der Hulst, J. M., Crane, P. C., \& Kennicutt, R. C. 1988, ApJ, 330, 667

Tully, R. B. 1974, ApJS, 27, 449

van der Hulst, J. M., Terlouw, J. P., Begeman, K. G., Zwitser, W., \& Roelfsema, P. R. 1992, in ASP Conf. Ser. 25, Astronomical Data Analysis Software and Systems I, ed. D. M. Worrall, C. Biemesderfer, \& J. Barnes (San Francisco, CA: ASP), 131

van Dishoeck, E. F., \& Black, J. H. 1988, ApJ, 334, 771

Visser, R., van Dishoeck, E. F., \& Black, J. H. 2009, A\&A, 503, 323

Vogel, S. N., Kulkarni, S. R., \& Scoville, N. Z. 1988, Natur, 334, 402

Vogel, S. N., Rand, R. J., Gruendl, R. A., \& Teuben, P. J. 1993, PASP, 105,666

Vogelaar, M. G. R., \& Terlouw, J. P. 2001, in ASP Conf. Ser. 238, Astronomical Data Analysis Software and Systems X, ed. F. R. Harnden, Jr., F. A. Primini, \& H. E. Payne (San Francisco, CA: ASP), 358

Walter, F., Brinks, E., de Blok, W. J. G., et al. 2008, AJ, 136, 2563

Wei, L. H., Keto, E., \& Ho, L. C. 2012, ApJ, 750, 136

Weiss, A., Neininger, N., Hüttemeister, S., \& Klein, U. 2001, A\&A, 365, 571

Whitmore, B. C., Chandar, R., Kim, H., et al. 2011, ApJ, 729, 78

Wiebe, D. S., Egorov, O. V., \& Lozinskaya, T. A. 2011, ARep, 55, 585

Wilson, C. D., Walker, C. E., \& Thornley, M. D. 1997, ApJ, 483, 210

Wolfire, M. G., Tielens, A. G. G. M., Hollenbach, D., \& Kaufman, M. J. 2008, ApJ, 680, 384

Young, J. S., \& Scoville, N. Z. 1991, ARA\&A, 29, 581

Zaritsky, D., Rix, H.-W., \& Ricke, M. 1993, Natur, 364, 313 